\title{
FIXED POINTS AND COMPLETENESS IN METRIC AND IN GENERALIZED METRIC SPACES
}

\author{
S. COBZAŞ
}

\begin{abstract}
The famous Banach Contraction Principle holds in complete metric spaces, but completeness is not a necessary condition - there are incomplete metric spaces on which every contraction has a fixed point. The aim of this paper is to present various circumstances in which fixed point results imply completeness. For metric spaces this is the case of Ekeland variational principle and of its equivalent - Caristi fixed point theorem. Other fixed point results having this property will be also presented in metric spaces, in quasi-metric spaces and in partial metric spaces. A discussion on topology and order and on fixed points in ordered structures and their completeness properties is included as well.
\end{abstract}

AMS 2010 MSC:

Primary: $47 \mathrm{H} 10$

Secondary: 47H04 54C60 54E15 54E35 06B30 06F30

Key words: fixed point; variational principles; completeness; partially ordered sets; topological space; set-valued mapping; metric space; generalized metric; partial-metric; quasi-metric

Motto:

All roads lead to Rome - Caesar Augustus. 1

All topologies come from generalized metrics - Ralph Kopperman?2.

\section{Contents}

Introduction

1. Banach contraction principle in metric spaces 2

1.1. Contractions and contractive mappings 3

1.2. Converses of Banach's contraction principle 4

1.3. Neither completeness nor compactness is necessary 7

1.4. Completeness and other properties implied by FPP 8

2. Variational principles and completeness 15

2.1. Ekeland Variational Principle

2.2. The strong Ekeland principle, compactness and reflexivity 18

3. Other principles $\quad 24$

3.1. Takahashi minimization principle 24

3.2. Dancs-Hegedüs-Medvegyev principle 25

3.3. Arutyunov's principle 26

3.4. Weak sharp minima and completeness 28

4. EkVP in quasi-metric spaces 31

4.1. Quasi-metric spaces 31

4.2. Completeness in quasi-metric spaces 32

Date: February 08, 2018.

${ }^{1}$ see Wikipedia, Milliarium Aureum.

${ }^{2}$ Amer. Math. Monthly 95 (1988), no. 2, 89-97, 121. 
4.3. Variational principles, fixed points and completeness 33

4.4. Smyth completeness 38

4.5. Some results of Bao, Cobzaş, Mordukhovich and Soubeyran 39

5. Topology and order $\quad 43$

5.1. Partially ordered sets 43

5.2. Order relations in topological spaces 44

5.3. Topologies on ordered sets - Alexandrov's, the upper topology, Scott's, the interval topology

6. Fixed points in partially ordered sets 51

6.1. Fixed point theorems

6.2. Converse results $\quad 52$

6.3. Fixed points in ordered metric spaces

7. Partial metric spaces $\quad 56$

7.1. Definition and topological properties

7.2. Convergent sequences, completeness and the Contraction Principle 60

7.3. Topology and order on partial metric spaces $\quad 63$

7.4. The specialization order in quasi-metric spaces $\quad 65$

7.5. Partial metrics and quasi-metrics $\quad 65$

7.6. The existence of suprema in partial metric spaces 68

7.7. Caristi's fixed point theorem and completeness in partial metric spaces 69

7.8. Ekeland Variational Principle (EkVP) in partial metric spaces

7.9. Dislocated metric spaces $\quad 76$

7.10. Other generalized metric spaces $\quad 78$

Appendix - A pessimistic conclusion $\quad 80$

References 81

\section{INTRODUCTION}

The famous Banach Contraction Principle holds in complete metric spaces, but completeness is not a necessary condition - there are incomplete metric spaces on which every contraction has a fixed point, see, e.g., 65. The aim of the present paper is to present various circumstances in which fixed point results imply completeness. For metric spaces this is the case of Ekeland variational principle (and of its equivalent - Caristi fixed point theorem) (see, for instance, the surveys [35], [127], [190]) but this is also true in quasimetric spaces ([47], 104]) and in partial metric spaces ([3], [170]). Other fixed point results having this property will be also presented.

Various order completeness conditions of some ordered structures implied by fixed point properties will be considered as well.

Concerning proofs - in several cases we give proofs, mainly to the converse results, i.e. completeness implied by fixed point results. In Sections 5 we give full proofs to results relating topology and order as well as in Section 7 in what concerns the properties of partial metric spaces.

\section{BANACH CONTRACtion PRINCIPLE IN METRIC SPACES}

Banach Contraction Principle was proved by S. Banach in his thesis from 1920, published in 1922, 29]. Although the idea of successive approximations in some concrete situations (solving differential and integral equations) appears in some works of E. Picard, 
R. Caccioppoli, et al., it was Banach who placed it in the right abstract setting, making it suitable for a wide range of applications (see the expository paper [113]).

1.1. Contractions and contractive mappings. Let $(X, \rho)$ and $(Y, d)$ be metric spaces. A mapping $f: X \rightarrow Y$ is called Lipschitz if there exists a number $\alpha \geq 0$ such that

$$
\forall x, y \in X, \quad d(f(x), f(y)) \leq \alpha \rho(x, y) .
$$

The number $\alpha$ is called a Lipschitz constant for $f$, and one says sometimes that the mapping $f$ is $\alpha$-Lipschitz. If $\alpha=0$, then the mapping $f$ is constant $f(x)=f\left(x_{0}\right)$ for some point $x_{0} \in X$. If $\alpha=1$, i.e.

$$
\forall x, y \in X, \quad d(f(x), f(y)) \leq \rho(x, y),
$$

then the mapping $f$ is called nonexpansive. If

$$
\forall x, y \in X, \quad d(f(x), f(y))=\rho(x, y),
$$

then $f$ is called an isometry.

Suppose now $Y=X$. An $\alpha$-Lipschitz mapping $f: X \rightarrow X$ with $0 \leq \alpha<1$ is called a contraction. A mapping $f: X \rightarrow X$ satisfying the relation

$$
\forall x, y \in X, x \neq y, \quad \rho(f(x), f(y))<\rho(x, y),
$$

is called contractive.

A point $x_{0} \in X$ such that $f\left(x_{0}\right)=x_{0}$ is called a fixed point of the mapping $f: X \rightarrow X$. The study of the fixed points of mappings is one of the most important branches of mathematics, with numerous applications to the solution of various kinds of equations (differential, integral, partial differential, operator), optimization, game theory, etc.

The following theorem is, perhaps, the most known fixed point result.

Theorem 1.1 (Banach's Contraction Principle). Any contraction on a complete metric space has a fixed point.

More exactly, suppose that for some $\alpha, 0 \leq \alpha<1, f$ is an $\alpha$-contraction on a complete metric space $(X, \rho)$. Then, for an arbitrary point $x_{1} \in X$, the sequence $\left(x_{n}\right)$ defined by the recurrence relation

$$
x_{n+1}=f\left(x_{n}\right), n \in \mathbb{N},
$$

converges to a fixed point $x_{0}$ of the mapping $f$, and the following estimations hold:

(a) $\forall n \in \mathbb{N}, \quad \rho\left(x_{n}, x_{n+1}\right) \leq \alpha^{n-1} \rho\left(x_{1}, x_{2}\right)$;

(b) $\forall n \in \mathbb{N}, \forall k \in \mathbb{N}, \quad \rho\left(x_{n}, x_{n+k}\right) \leq \frac{1-\alpha^{k}}{1-\alpha} \alpha^{n-1} \rho\left(x_{1}, x_{2}\right)$;

(c) $\forall n \in \mathbb{N}, \quad \rho\left(x_{n}, x_{0}\right) \leq \frac{\alpha^{n-1}}{1-\alpha} \rho\left(x_{1}, x_{2}\right)$.

Under a supplementary condition contractive mappings also have fixed points.

Theorem 1.2 (M. Edelstein (1962) [61, 62]). Let $(X, \rho)$ be a metric space and $f: X \rightarrow X$ a contractive mapping. If there exists $x \in X$ such that the sequence of iterates $\left(f^{n}(x)\right)$ has a limit point $\xi \in X$, then $\xi$ is the unique fixed point of $f$.

Theorem 1.2 has the following important consequence. 
Corollary 1.3 (Nemytskii (1936) [146]). If the metric space $(X, \rho)$ is compact, then every contractive mapping $f: X \rightarrow X$ has a unique fixed point in $X$.

Moreover, for any $x_{1} \in X$ the sequence defined by $x_{n+1}=f\left(x_{n}\right), n \in \mathbb{N}$, converges to the fixed point of the mapping $f$.

Fixed point results for isometries were proved by Edelstein in 63 .

1.2. Converses of Banach's contraction principle. Supposing that a function $f$ acting on a metric space $(X, \rho)$ has a unique fixed point one looks for conditions ensuring the existence of a metric $\bar{\rho}$ on $X$, topologically equivalent to $\rho$ such that $f$ is a contraction on $(X, \bar{\rho})$. The first result of this kind was obtained by Bessaga [37. Good presentations of various aspects of fixed points for contraction mappings and their generalizations as well as converse-type results are contained in Ivanov 88, Lahiri et al. [123, Kirk [113, Opoitsev [156], Rus 176, Rus [174, Rus and Petruşels [179].

We say that a metric $d$ on a set $X$ is complete if $(X, d)$ is a complete metric space.

Theorem 1.4 (Cz. Bessaga (1959) [37]). Let $X$ be a nonempty set, $f: X \rightarrow X$ and $\alpha \in(0,1)$.

(1) If for every $n \in \mathbb{N}, f^{n}$ has at most one fixed point, then there exists a metric $\rho$ on $X$ such that $f$ is an $\alpha$-contraction with respect to $\rho$.

(2) If, in addition, some $f^{n}$ has a fixed point, then there exists a complete metric $\rho$ on $X$ such that $f$ is an $\alpha$-contraction with respect to $\rho$.

A different proof of Theorem 1.4 was given Wong [219], a version of which is included in Deimling's book on nonlinear functional analysis, [55, p. 191-192]. Other proofs as well as some extensions were given by Babu [28, Jachymski [93. (see also [89]), Palczewski and Miczko [158, 159], Wang et al. [214] (cf. the MR review). Angelov [17, 18, proved a converse result in the context of uniform spaces.

In the case of compact metric spaces Janoš [99] proved the following result.

Theorem 1.5. Let $(X, \rho)$ be a compact metric space and $f: X \rightarrow X$ be a continuous mapping such that, for some $\xi \in X$,

$$
\bigcap_{n=1}^{\infty} f^{n}(X)=\{\xi\} .
$$

Then for every $\alpha \in(0,1)$ there exists a metric $\rho_{\alpha}$ on $X$, topologically equivalent to $\rho$, such that $f$ is an $\alpha$-contraction with respect to $\rho_{\alpha}$ (with $\xi$ as the unique fixed point).

A mapping $f$ satisfying (1.7) is called squeezing.

Another proof of Janoš' theorem was given by Edelstein [64].

Kasahara [106 showed that compactness is also necessary for the validity of Janoš' result.

Theorem 1.6. Let $(X, \rho)$ be a metric space. If for every squeezing mapping $f: X \rightarrow X$ and every $\alpha \in(0,1)$ there exits a metric $\rho_{\alpha}$ on $X$, topologically equivalent to $\rho$, such that $f$ is an $\alpha$-contraction with respect to $\rho_{\alpha}$, then the space $X$ is compact.

Janoš extended in [100] this result to uniform spaces (more exactly, to completely regular spaces whose topology is generated by a family of semimetrics), see also Angelov [16, 17, [18, 19]. Rus [175] extended Janoš' result to weakly Picard mappings. An operator $f$ on a metric space $(X, \rho)$ is called weakly Picard if, for every $x \in X$, the sequence $\left(f^{n}(x)\right)_{n=1}^{\infty}$ 
of iterates converges to a fixed point of $f$. If further, the limit is independent of $x$ (i.e. $f$ has a unique fixed point), then $f$ is called a Picard operator (see [177] or [179]).

Other extensions of Janoš' result were given by Leader [124] (see also Leader [125, 126]), Meyers [143, 144, Mukherjee and Som [145]. For a metric space $(X, \rho)$ and $\xi \in X$ consider the following properties:

(i) $f^{n}(x) \rightarrow \xi$ for every $x \in X$;

(ii) the convergence in (i) is uniform on some neighborhood $U$ of $\xi$.

The condition (ii) means that

$$
\forall \varepsilon>0, \exists n_{0}=n_{0}(\varepsilon) \text {, such that } \forall n \geq n_{0}, f^{n}(U) \subseteq B[\xi, \varepsilon] .
$$

To designate the uniform convergence on a subset $A$ of $X$ of the sequence $\left(f^{n}\right)$ to a point $\xi$, one uses the notation

$$
f^{n}(A) \rightarrow \xi
$$

Leader [124] proved the following results.

Theorem 1.7. Let $(X, \rho)$ be a metric space and $f: X \rightarrow X$.

(1) There exists a metric $\bar{\rho}$ topologically equivalent to $\rho$ on $X$ such that $f$ is a Banach contraction under $\bar{\rho}$ with fixed point $\xi$ if, and only if, $f$ is continuous and both (i) and (ii) from (1.8) hold.

(2) There exists a bounded metric $\bar{\rho}$ topologically equivalent to $\rho$ on $X$ such that $f$ is a Banach contraction under $\bar{\rho}$ with fixed point $\xi$ if, and only if, $f$ is continuous and $f^{n}(X) \rightarrow \xi$

(3) There exists a bounded metric $\bar{\rho}$ uniformly equivalent to $\rho$ on $X$ such that $f$ is a Banach contraction under $\bar{\rho}$ if, and only if, $f$ is uniformly continuous and

$$
\operatorname{diam}_{\rho}\left(f^{n}(X)\right) \rightarrow 0 \text { as } n \rightarrow \infty .
$$

We mention also a topological characterization of contractions given by Florinskij [66].

Theorem 1.8. Let $(X, \tau)$ be a metrizable topological space and $f: X \rightarrow X$ a continuous mapping. The following are equivalent.

(1) There exists a metric $\rho$ on $X$ generating the topology $\tau$ such that $f$ is a Banach contraction with respect to $\rho$.

(2) There exists a nonempty open subset $G$ of $X$ such that

(i) $f(G) \subseteq G$;

(ii) $\operatorname{diam}\left(\bigcap_{n=1}^{\infty} \overline{f^{n}(G)}\right)=0 \quad$ (with the convention that $\operatorname{diam} \emptyset=0$ );

(iii) for every $x \in X$ there exists $n_{x} \in \mathbb{N}$ such that $f^{n}(x) \in G$ for all $n \geq n_{x}$.

Remark 1.9. Florinskij [66] gave also topological characterizations of mappings $f: X \rightarrow$ $X$ that are:

- nonexpansive, i.e. $\rho(f(x), f(y)) \leq \rho(x, y), x, y \in X$;

- contractive, i.e. $\rho(f(x), f(y))<\rho(x, y), x, y \in X$;

- separating, meaning that $\rho(f(x), f(y)) \geq \alpha \rho(x, y), x, y \in X$, for some $\alpha \in(0,1)$.

The paper [66] contains no proofs and I did not find them elsewhere.

A general remetrization result was obtained by Florinskij [68, guaranteing that every contraction has a fixed point (without completeness). 
Theorem 1.10. On every metric space $(X, \rho)$ there exists a metric $\bar{\rho}$, topologically equivalent to $\rho$, such that the Banach Contraction Principle holds in the space $(X, \bar{\rho})$, i.e., every contraction on $(X, \bar{\rho})$ has a fixed point.

The proof is based on the following result, proved in the same paper.

Theorem 1.11. For every metric space $(X, \rho)$ there exists a metric space $(\tilde{X}, \tilde{\rho})$, containing $(X, \rho)$ as a dense subspace, such that the space $(\tilde{X}, \tilde{\rho})$ is connected, locally connected and uniformly chainable.

Let $(X, \rho)$ be a metric space and $\varepsilon>0$. An $\varepsilon$-chain joining two points $x, y \in X$ is a set $x=x_{1}, x_{2}, \ldots, x_{n}=y$ of points in $X$ such that $d\left(x_{i}, x_{i+1}\right) \leq \varepsilon$ for $i=1,2, \ldots, n-1$. The metric space $(X, \rho)$ is called chainable if for every $x, y \in X$ and $\varepsilon>0$ there exists an $\varepsilon$-chain connecting $x$ and $y$, and uniformly chainable if for every $\varepsilon>0$ there exists $n(\varepsilon) \in \mathbb{N}$ such that any pair $x, y$ of points in $X$ can be joined by an $\varepsilon$-chain containing at most $n(\varepsilon)$ elements.

The following curious result was also obtained by Florinskij [67].

Theorem 1.12. Let $(X, \rho)$ be a separable and uniformly chainable metric space. Then there exists a metric $\tilde{\rho}$ on $X$, uniformly equivalent to $\rho$, such that every contraction on $(X \tilde{\rho})$ is a constant mapping, i.e. applies $X$ in a single point.

\section{Ultrametric spaces}

In the case of an ultrametric space, the situation is simpler. An ultrametric space is a metric space $(X, \rho)$ such that $\rho$ satisfies the so called strong triangle (or ultrametric) inequality

$$
\rho(x, z) \leq \max \{\rho(x, y), \rho(y, z)\}
$$

for all $x, y, z \in X$.

Bellow we present some specific properties of these spaces.

Proposition 1.13. Let $(X, \rho)$ be an ultrametric space. Then for all $x, y, z \in X$ and $r>0$,

(i) $\rho(x, y) \neq \rho(y, z) \Longrightarrow \rho(x, z)=\max \{\rho(x, y), \rho(y, z)\}$;

(ii) $y \in B[x, r] \Longrightarrow B[x, r]=B[y, r]$;

(iii) $r_{1} \leq r_{2}$ and $B\left[x, r_{1}\right] \cap B\left[x, r_{2}\right] \neq \emptyset \Longrightarrow B\left[x, r_{1}\right] \subseteq B\left[x, r_{2}\right]$.

Similar relations hold for the open balls $B(x, r)$.

Remark 1.14. These strange properties of non-Archimedean metric spaces remind us of the following Pascal's thought:

Nature is an infinite sphere of which the center is everywhere and the circumference nowhere (Blaise Pascal, Pensées).

Probably the famous French philosopher and mathematician had in mind a non-Archimedean world. Initially he even wrote "A frightful (effroyable) sphere".

See also the essay on this topic by J. L. Borges at

http://www.filosofiaesoterica.com/pascals-sphere/

An ultrametric space $(X, \rho)$ is called spherically complete if for every collection $B_{i}=$ $B\left[x_{i}, r_{i}\right], i \in I$, of closed ball in $X$ such that $B_{i} \cap B_{j} \neq \emptyset$ for all $i, j \in I$, has nonempty intersection, $\bigcap_{i \in I} B_{i} \neq \emptyset$. It is obvious that a spherically complete ultrametric space is complete. In an arbitrary metric space this property is called the binary intersection property. 
Priess-Crampe [168] proved the following converse to Edelstein's theorem on contractive mappings.

Theorem 1.15. An ultrametric space $(X, \rho)$ is spherically complete if and only if every contractive mapping on $X$ has a (unique) fixed point.

Remark 1.16. In fact Priess-Crampe 168 proved this result in the more general context of an ultrametric $\rho$ taking values in a totally ordered set $\Gamma$ having a least element 0 such that $0<\gamma$ for all $\gamma \in \Gamma$.

Fixed point theorems for contractive and for nonexpansive mappings on spherically complete non-Archimedean normed spaces were proved by Petalas and Vidalis [163.

Concerning contractions we mention the following result obtained by Hitzler and Seda 81.

Theorem 1.17. Let $(X, \tau)$ be a $T_{1}$ topological space and $f: X \rightarrow X$ a function on $X$. The following are equivalent:

(1) (i) The mapping $f$ has a unique fixed point $\xi \in X$, and

(ii) for every $x \in X$ the sequence $\left(f^{n}(x)\right)$ converges to $\xi$ with respect to the topology $\tau$.

(2) There exists a complete ultrametric $\rho$ on $X$ such that $\rho(f(x), f(y)) \leq 2^{-1} d(x, y)$ for all $x . y \in X$.

For applications of these fixed point results to logic programming see the paper [82].

Remark 1.18. It is not sure that the metric $\rho$ from (2) generates the topology $\tau$, but for every $x \in X$ the sequence $\left(f^{n}(x)\right)$ converges to $\xi$ with respect to the topology $\tau$ and the metric $\rho$.

1.3. Neither completeness nor compactness is necessary. In this subsection we shall provide some examples of peculiar topological spaces having the fixed point property (FPP) for various classes of mappings.

Examples 1.19 (Elekes 65]).

1. The space $X=\{(x, \sin (1 / x)): x \in(0,1]\}$ is a non-closed (hence incomplete) subset of $\mathbb{R}^{2}$ having the FPP for contractions (Theorem 1.2).

2. For every $n \in \mathbb{N}$ every open subset of $\mathbb{R}^{n}$ possessing the Banach Fixed Point Property coincides with $\mathbb{R}^{n}$, hence is closed (Corollary 2.2).

3. Every simultaneously $F_{\sigma}$ and $G_{\delta}$ subset of $\mathbb{R}$ with the Banach Fixed Point Property is closed (Theorem 2.4).

4. There exists a nonclosed $G_{\delta}$ set $X \subseteq \mathbb{R}$ with the Banach Fixed Point Property. Moreover, $X \subseteq[0,1]$ and every contraction mapping of $X$ into itself is constant (Theorem $3.3)$.

5. There exists a nonclosed $F_{\sigma}$ subset of $[0,1]$ with the Banach Fixed Point Property (Theorem 3.4).

6. There is a bounded Borel (even $F_{\sigma}$ ) subset of $\mathbb{R}$ with the Banach Fixed Point Property that is not complete with respect to every equivalent metric (Corollary 3.5).

7. For every integer $n>0$ there exists a nonmeasurable set in $\mathbb{R}^{n}$ with the Banach Fixed Point Property (Theorem 3.6).

The example from 1 was presented at the Problem Session of the 34th Winter School in Abstract Analysis, Lhota nad Rohanovem, Czech Republic, 2006, by E. Behrends, 
classified by him as "folklore", along with some questions concerning the subsets of $\mathbb{R}^{n}$ (in particular of $\mathbb{R}$ ) having Banach Fixed Point Property (i.e. FPP for contractions).

We give the proof only for 1 , following [65]. A proof based on some similar ideas was given by Borwein [39].

Proof of the assertion 1. Let $X=\{(x, \sin (1 / x)): x \in(0,1]\}$ and $f: X \rightarrow X$ be a contraction with constant $0<\alpha<1$. For $H \subseteq(0,1]$ put $X_{H}:=\{(x, y) \in X: x \in H\}$.

Let $0<\varepsilon<1$ be such that $\alpha \sqrt{\varepsilon^{2}+4}<2$. Then for all $z=(x, y), z^{\prime}=\left(x^{\prime}, y^{\prime}\right)$ in $X$ with $0<x, x^{\prime}<\varepsilon$,

$$
\left\|f(z)-f\left(z^{\prime}\right)\right\| \leq \alpha \sqrt{\left(x-x^{\prime}\right)^{2}+\left(y-y^{\prime}\right)^{2}}<\alpha \sqrt{\varepsilon^{2}+4}<2 .
$$

Consequently, $X_{(0, \varepsilon)}$ does not contain both a local minimum and a local maximum of the graph. Since $X_{(0, \varepsilon)}$ is connected, it follows that it is contained in at most two consecutive monotone parts of the graph of $\sin (1 / x)$. Therefore there exists $\delta_{1}>0$ such that $f\left(X_{(0, \varepsilon)}\right) \subseteq X_{\left[\delta_{1}, 1\right]}$ for some $\delta_{1}>0$. By compactness $f\left(X_{[\varepsilon, 1]}\right) \subseteq X_{\left[\delta_{2}, 1\right]}$ for some $\delta_{2}>0$.

Taking $\delta=\min \left\{\delta_{1}, \delta_{2}\right\}$ it follows $f(X) \subseteq X_{[\delta, 1]}$ and so $f\left(X_{[\delta, 1]}\right) \subseteq X_{[\delta, 1]}$. Applying Banach Fixed Point Theorem to $X_{[\delta, 1]}$ it follows that $f$ has a fixed point.

Some examples of spaces having the FPP for continuous mappings were given by Connell 52. These examples show that, in author's words: "in the general case, compactness and the FPP are only vaguely related".

We first mention the following result of Klee.

Theorem 1.20 (Klee [116]). A locally connected, locally compact metric space with the FPP for continuous mappings is compact.

Examples 1.21. (Connell [52])

1. There exists a Hausdorff topological space $X$ having the FPP for continuous mappings such that the only compact subsets of $X$ are the finite ones.

2. There exists a metric space $X$ having the FPP for continuous mappings such that $X^{2}$ does not have the FPP for continuous mappings.

3. There exists a separable, locally contractible metric space that has the FPP for continuous mappings, yet it is not compact.

4. There exists a compact metric space $X$ that does not have the FPP for continuous mappings, yet it contains a dense subset $Y$ that does have the FPP for continuous mappings.

1.4. Completeness and other properties implied by FPP. We shall present some fixed point results that imply the completeness of the underlying space. The papers [35, [127] and [190] contain surveys on this topic. A good analysis is given in the Master Thesis of Nicolae [147].

We first mention the following characterization of the field of real numbers among totally ordered fields.

Suppose $R$ is an ordered field. Call a continuous map $f: R \rightarrow R$ a contraction if there exists $r<1$ (in $R$ ) such that $|f(x)-f(y)| \leq r|x-y|$ for all $x, y \in R$ (where $|x|:=\max \{x,-x\})$.

The following result is taken from

http://mathoverflow.net/questions/65874/converse-to-banach-s-fixed-point-theorem-forordered-fields

Asking a question posed by James Propp, George Lowther proved the following result. 
Theorem 1.22. If $R$ is an ordered field such that every contraction on $R$ has a fixed point, then $R \cong \mathbb{R}$.

The proof is done in two steps:

I. one shows first that the order of $R$ is Archimedean, and then

II. one proves that every Cauchy sequence is convergent (i.e. the completeness of $R$ ), two properties that characterize the field $\mathbb{R}$ among the ordered fields.

The first characterization of completeness in terms of contraction was done by $\mathrm{Hu}$ [85].

Theorem 1.23. A metric space $(X, \rho)$ is complete if and only if for every nonempty closed subset $Y$ of $X$ every contraction on $Y$ has a fixed point in $Y$.

Proof. The idea of the proof is simple. One takes a Cauchy sequence $\left(x_{n}\right)$ in $X$. If it has a convergent subsequence, then it converges. Supposing that this is not the case, then $\beta\left(x_{n}\right):=\inf \left\{\rho\left(x_{n}, x_{m}\right): m>n\right\}>0$ for all $n \in \mathbb{N}$. For a given $\alpha$ with $0<\alpha<1$, one constructs inductively a subsequence $\left(x_{n_{k}}\right)$ such that $\rho\left(x_{i}, x_{j}\right) \leq \alpha \beta\left(x_{n_{k-1}}\right)$ for all $i, j \geq n_{k}$. Then $Y=\left\{x_{n_{k}}: k \in \mathbb{N}\right\}$ is a closed subset of $X$ and the function $f\left(x_{n_{k}}\right)=x_{n_{k+1}}, k \in \mathbb{N}$, is an $\alpha$-contraction on $Y$, because

$$
\rho\left(f\left(x_{n_{k}}\right), f\left(x_{n_{k+i}}\right)\right)=\rho\left(x_{n_{k+1}}, x_{n_{k+i+1}}\right) \leq \alpha \beta\left(x_{n_{k}}\right) \leq \alpha \rho\left(x_{n_{k}}, x_{n_{k+i}}\right),
$$

for all $k, i \in \mathbb{N}$. It is obvious that $f$ has no fixed points.

Subrahmanyam [188] proved the following completeness result.

Theorem 1.24. A metric space $(X, \rho)$ in which every mapping $f: X \rightarrow X$ satisfying the conditions

(i) there exists $\alpha>0$ such that $\rho(f(x), f(y)) \leq \alpha \max \{\rho(x, f(x)), \rho(y, f(y))\}$ for all $x, y \in X$;

(ii) $f(X)$ is countable;

has a fixed point, is complete.

The condition (i) in this theorem is related to Kannan and Chatterjea conditions: there exists $\alpha \in\left(0, \frac{1}{2}\right)$ such that for all $x, y \in X$,

$$
\rho(f(x), f(y)) \leq \alpha[\rho(x, f(x))+\rho(y, f(y))],
$$

respectively

$$
\rho(f(x), f(y)) \leq \alpha[\rho(x, f(y))+\rho(y, f(x))] .
$$

Kannan and Chatterjea proved that any mapping $f$ on a complete metric space satisfying $(\overline{\mathrm{K}})$ or $(\mathrm{Ch})$ has a fixed point (see, for instance, [179]). As it is remarked in [188 Theorem 1.24 provides completeness of metric spaces on which every Kannan, or every Chatterjea map, has a fixed point.

Another case when the fixed point property for contractions implies completeness was discovered by Borwein 39 .

A metric space $(X, \rho)$ is called uniformly Lipschitz connected if there exists $L \geq 0$ such that for any pair $x_{0}, x_{1}$ of points in $X$ there exists a mapping $g:[0,1] \rightarrow X$ such that $g(0)=x_{0}, g(1)=x_{1}$ and

$$
\rho(g(s), g(t)) \leq L|s-t| \rho(g(0), g(1)),
$$

for all $s, t \in[0,1]$. 
Obviously, a convex subset $C$ of a normed space $X$ is uniformly Lipschitz connected, the mapping $g$ connecting $x_{0}, x_{1} \in C$ being given by $g(t)=(1-t) x_{0}+t x_{1}, t \in[0,1]$. In this case

$$
\|g(s)-g(t)\|=|s-t|\left\|x_{1}-x_{0}\right\|
$$

for all $s, t \in[0,1]$.

From the following theorem it follows that a convex subset $C$ of a normed space $X$ is complete if and only if any contraction on $C$ has a fixed point. In particular this holds for the normed space $X$.

Theorem 1.25. Let $C$ be a uniformly Lipschitz connected subset of a complete metric space $(X, \rho)$. Then the following conditions are equivalent.

(1) The set $C$ is closed.

(2) Every contraction on $C$ has a fixed point.

(3) Any contraction on $X$ which leaves $C$ invariant has a fixed point in $C$.

Proof. The implication (1) $\Rightarrow(2)$ is Banach Fixed Point Theorem and $(2) \Rightarrow(3)$ is obvious.

It remains to prove $(3) \Rightarrow(1)$. Supposing that $C$ not closed, there exists a point $\bar{x} \in \bar{C} \backslash C$. Let $\left(x_{k}\right)_{k \in \mathbb{N}_{0}}$ be a sequence of pairwise distinct points in $C$ such that

$$
\rho\left(x_{k}, \bar{x}\right) \leq \min \left\{\frac{1}{2^{k+4}}, \frac{L}{2^{k+4}}\right\},
$$

for $k=0,1, \ldots$, where $L>0$ is the constant given by the uniform Lipschitz connectedness of $C$.

It follows

$$
\rho\left(x_{k}, x_{k+1}\right) \leq \min \left\{\frac{1}{2^{k+3}}, \frac{L}{2^{k+3}}\right\}
$$

for all $k \in \mathbb{N}_{0}$.

Let $g_{k}:[0,1] \rightarrow C$ be such that $g_{k}(0)=x_{k}, g_{k}(1)=x_{k+1}$ and

$$
\rho\left(g_{k}(s), g_{k}(t)\right) \leq L|s-t| \rho\left(x_{k}, x_{k+1}\right),
$$

for all $s, t \in[0,1]$. Define $g:(0, \infty) \rightarrow C$ by

$$
g(t)= \begin{cases}x_{0} & \text { for } 1<t<\infty, \\ g_{k}\left(2^{k+1} t-1\right) & \text { for } \frac{1}{2^{k+1}}<t \leq \frac{1}{2^{k}} .\end{cases}
$$

It follows $g\left(2^{-k}\right)=g_{k}(1)=x_{k+1}$.

Let $\Delta_{k}=\left(2^{-(k+1)}, 2^{-k}\right]$. Then for $s, t \in \Delta_{k}$, taking into account (1.15) and (1.14), one obtains

$$
\begin{aligned}
\rho(g(s), g(t)) & \leq L \cdot 2^{k+1}|s-t| \rho\left(x_{k}, x_{k+1}\right) \\
& \leq L \cdot 2^{k+1} \cdot|s-t| \cdot \frac{1}{2^{k+3}}=\frac{L}{4} \cdot|s-t| \leq L \cdot|s-t| .
\end{aligned}
$$

Since $|s-t|<\frac{1}{2^{k+1}}$, it follows also that

$$
\rho(g(s), g(t)) \leq L \cdot 2^{k+1} \cdot \frac{1}{2^{k+1}} \cdot \frac{1}{2^{k+3}}=\frac{L}{2^{k+3}},
$$

for all $s, t \in \Delta_{k}$. 
If $s \in \Delta_{k}$ and $t \in \Delta_{p}$ with $k \leq p$, then the above inequality and (1.13) yield

$$
\begin{aligned}
\rho\left(g(s), g\left(2^{-k}\right)\right) & \leq \frac{L}{2^{k+3}} ; \\
\rho\left(x_{k+1}, x_{p+1}\right) & \leq \rho\left(x_{k+1}, \bar{x}\right)+\rho\left(\bar{x}, x_{p+1}\right) \leq L\left(\frac{1}{2^{k+5}}+\frac{1}{2^{p+5}}\right) ; \\
\rho\left(g\left(2^{-p}\right), g(t)\right) & \leq \frac{L}{2^{p+3}},
\end{aligned}
$$

so that

$$
\begin{aligned}
\rho(g(s), g(t)) & \leq \rho\left(g(s), g\left(2^{-k}\right)\right)+\rho\left(x_{k+1}, x_{p+1}\right)+\rho\left(g\left(2^{-p}\right), g(t)\right) \\
& \leq L \cdot\left(\frac{1}{2^{k+3}}+\frac{1}{2^{k+5}}+\frac{1}{2^{p+5}}+\frac{1}{2^{p+3}}\right) .
\end{aligned}
$$

Observe that $s-t>\frac{1}{2^{k}}-\frac{1}{2^{p+1}}$, and so if we show that

$$
\frac{1}{2^{k+3}}+\frac{1}{2^{k+5}}+\frac{1}{2^{p+5}}+\frac{1}{2^{p+3}} \leq \frac{1}{2^{k}}-\frac{1}{2^{p+1}},
$$

then

$$
\rho(g(s), g(t)) \leq L|s-t| .
$$

Since all the fractions with $p$ at the denominator are less or equal to the corresponding ones with $k$ at the denominator, it follows

$$
\begin{aligned}
& \frac{1}{2^{k+3}}+\frac{1}{2^{k+5}}+\frac{1}{2^{p+5}}+\frac{1}{2^{p+3}}+\frac{1}{2^{p+1}} \\
& \leq \frac{1}{2^{k+2}}+\frac{1}{2^{k+4}}+\frac{1}{2^{k+1}}=\frac{13}{2^{k+4}}<\frac{1}{2^{k}},
\end{aligned}
$$

so that (1.17) holds.

Put now $g(0)=\bar{x}$. If $t \in \Delta_{k}$, then

$$
\begin{aligned}
\rho(g(0), g(t)) & \leq \rho\left(\bar{x}, x_{k+1}\right)+\rho\left(x_{k+1}, g(t)\right) \\
& \leq L\left(\frac{1}{2^{k+5}}+\frac{1}{2^{k+3}}\right)<L \cdot \frac{1}{2^{k+1}}<L \cdot t,
\end{aligned}
$$

showing that $g$ satisfies (1.18) for all $s, t \in[0, \infty)$. Let $h: X \rightarrow[0, \infty)$ and $f: X \rightarrow X$ be defined for $x \in X$ by

$$
h(x):=(2 L)^{-1} \rho(x, \bar{x}) \quad \text { and } \quad f(x):=(g \circ h)(x),
$$

respectively. Then, for all $x, x^{\prime} \in X$,

$$
\begin{aligned}
\rho\left(f(x), f\left(x^{\prime}\right)\right) & =\rho\left(g\left(\frac{1}{2 L} \rho(x, \bar{x})\right), g\left(\frac{1}{2 L} \rho\left(x^{\prime}, \bar{x}\right)\right)\right) \\
& \leq L \cdot \frac{1}{2 L}\left|\rho(x, \bar{x})-\rho\left(x^{\prime}, \bar{x}\right)\right| \leq \frac{1}{2} \cdot \rho\left(x, x^{\prime}\right),
\end{aligned}
$$

that is $f$ is a $\frac{1}{2}$-contraction on $X$. Because

$$
f(C)=g(h(C)) \subseteq g((0, \infty)) \subseteq C,
$$

it follows that $C$ is invariant for $f$. Since

$$
\bar{x}=g(0)=g(h(\bar{x}))=f(\bar{x}),
$$


it follows that the only fixed point of $f$ is $\bar{x}$, which does not belong to $C$, in contradiction to the hypothesis.

We mention the following consequences.

\section{Corollary 1.26.}

(1) A uniformly Lipschitz connected metric space $(X, \rho)$ is complete if and only if it has the fixed point property for contractions.

(2) A convex subset $C$ of a normed space $X$ is complete if and only if any contraction on $C$ has a fixed point. In particular this holds for the normed space $X$.

Proof. For (1) consider $X$ as a uniformly Lipschitz connected subset of its completion $\tilde{X}$. The results in (2) were discussed before Theorem 1.25 .

Example 1.27 (Borwein [39]). There is a starshaped non-closed subset of $\mathbb{R}^{2}$ having the fixed point property for contractions, but not for continuous functions.

One takes

$$
L_{k}=\operatorname{co}\left(\left\{(0,0),\left(1, \frac{1}{2^{k}}\right)\right\}\right), k \in \mathbb{N},
$$

and $C=\bigcup\left\{L_{k}: k \in \mathbb{N}\right\}$. Then $C$ is starshaped with respect to $(0,0)$ and non-closed, because $\operatorname{co}(\{(0,0),(1,0)\}) \subseteq \bar{C} \backslash C$. One shows that $C$ has the required properties, see 39 for details.

Xiang [220] completed and extended Borwein's results. Let $(X, \rho)$ be a metric space. By an arc we mean a continuous function $g: \Delta \rightarrow X$, where $\Delta$ is an interval in $\mathbb{R}$. An arc $g:(0,1] \rightarrow X$ is called semi-closed if

$$
\forall \varepsilon>0, \exists \delta>0 \text {, such that } \rho(g(s), g(t))<\varepsilon \text { for all } s, t \in(0, \delta) .
$$

The arc $g$ is called Lipschitz semi-closed if the mapping $g$ is Lipschitz and satisfies (1.19).

The metric space $(X, \rho)$ is called arcwise complete if for every semi-closed arc $g:(0,1] \rightarrow$ $X$ there exists the limit $\lim _{t \searrow 0} g(t)$. If this holds for every Lipschitz semi-closed arc $g:(0,1] \rightarrow X$, then $X$ is called Lipschitz complete.

Some examples, [220, Examples 1.1, 1.2 and 2.3], show that the arcwise completeness is weaker than the usual completeness even in an arcwise connected space, and so is Lipschitz completeness. It is obvious from the definitions that Lipschitz completeness is weaker than arcwise completeness.

A metric space $(X, \rho)$ is called locally arcwise connected (respectively, locally Lipschitz connected) if there exists $\delta>0$ such that any pair $x_{0}, x_{1}$ of points in $X$ with $\rho\left(x_{0}, x_{1}\right) \leq \delta$ can be linked by an arc (respectively by a Lipschitz arc).

Theorem 1.28 ([220], Theorems 3.1 and 3.2). Let $(X, \rho)$ be a metric space.

(1) If $(X, \rho)$ has the fixed point property for contractions, then $X$ is Lipschitz complete.

(2) If $(X, \rho)$ is locally Lipschitz connected, then $X$ has the fixed point property for contractions if and only if it is Lipschitz complete.

The above result have the following consequence (compare with Corollary 1.26 and Example 1.27).

Corollary 1.29 ([220]). A starshaped subset of a normed space has the fixed point property for contractions if and only if it is Lipschitz complete. 
This implies that the starshaped set considered in Example 1.27 is Lipschitz complete, in spite of the fact that it is not closed. This furnishes a further example of a non complete starshaped set that is Lipschitz complete.

One says that the metric space $(X, \rho)$ has the strong contraction property if every mapping $f: X \rightarrow X$ which is a contraction with respect to some metric $\bar{\rho}$ on $X$, uniformly equivalent to $\rho$, has a fixed point.

Theorem 1.30 ([220, Theorems 4.1 and 4.4). Let $(X, \rho)$ be a metric space.

(1) If $(X, \rho)$ has the strong contraction property, then $X$ is arcwise complete.

(2) If $(X, \rho)$ is locally arcwise connected, then $X$ has the strong contraction property if and only if it is arcwise complete.

Suzuki [196] found an extension of Banach contraction principle that implies completeness. He considered the function $\theta:[0,1) \rightarrow(1 / 2,1]$

$$
\theta(r)=\left\{\begin{array}{lll}
1 & \text { if } \quad 0 \leq r \leq(\sqrt{5}-1) / 2 \\
(1-r) r^{-2} & \text { if } \quad(\sqrt{5}-1) / 2 \leq r \leq 2^{-1 / 2} \\
(1+r)^{-1} & \text { if } \quad 2^{-1 / 2} \leq r<1
\end{array}\right.
$$

and proved the following fixed point result.

Theorem 1.31. Let $(X, \rho)$ be a complete metric space and $f: X \rightarrow X$.

(1) If there exists $r \in[0,1)$ such that

$$
\theta(r) d(x, f(x)) \leq d(x, y) \Longrightarrow d(f(x), f(y)) \leq r d(x, y)
$$

for all $x, y \in X$, then $f$ has a fixed point $\bar{x}$ in $X$ and $\lim _{n} f^{n}(x)=\bar{x}$ for every point $x \in X$.

(2) Moreover, $\theta(r)$ is the best constant in (1.21) for which the result holds, in the sense that for every $r \in[0,1)$ there exist a complete metric space $(X, \rho)$ and a function $f: X \rightarrow X$ without fixed points and such that

$$
\theta(r) d(x, f(x))<d(x, y) \Longrightarrow d(f(x), f(y)) \leq r d(x, y)
$$

for all $x, y \in X$.

Extensions of Suzuki fixed point theorem to partial metric spaces and to partially ordered metric spaces were given by Paesano and Vetro [157.

The converse result is the following one.

Theorem 1.32 ([196], Corollary 1$)$. For a metric space $(X, \rho)$ the following are equivalent.

(1) The space $(X, \rho)$ is complete.

(2) There exists $r \in(0,1)$ such that every mapping $f: X \rightarrow X$ satisfying

$$
\frac{1}{10000} d(x, f(x)) \leq d(x, y) \Longrightarrow d(f(x), f(y)) \leq r d(x, y)
$$

for all $x, y \in X$, has a fixed point.

It is clear that the function $\theta(r)$ given by (1.20) satisfies the equality $\lim _{r} \nearrow_{1} \theta(r)=1 / 2$. The critical case of functions acting on a subset $X$ of a Banach space $E$ satisfying the condition

$$
\frac{1}{2}\|x-f(x)\| \leq\|x-y\| \Longrightarrow\|f(x)-f(y)\| \leq\|x-y\|,
$$

for all $x, y \in X$ was examined by Suzuki [195]. Condition (1.24) was called condition (C) and the functions satisfying this condition are called generalized nonexpansive. It is clear 
that every nonexpansive mapping satisfies (1.24), but there are discontinuous functions satisfying (1.24), so that the class of generalized nonexpansive mappings is strictly larger than that of nonexpansive ones. The term generalized nonexpansive is justified by the fact that the generalized nonexpansive mappings share with nonexpansive mappings several properties concerning fixed points - in some Banach spaces $E$ they have fixed points on every weakly compact convex subset of $E$, and for every closed bounded convex subset $X$ of $E$ and every generalized nonexpansive mapping $f$ on $X$ there exists an almost fixed point sequence, i.e. a sequence $\left(x_{n}\right)$ in $X$ such that $\left\|x_{n}-f\left(x_{n}\right)\right\| \rightarrow 0$ as $n \rightarrow \infty$, see 195. Also a generalized nonexpansive mapping $f$ is quasi-nonexpansive, in the sense that $\|f(x)-y\| \leq\|x-y\|$ for all $x \in X$ and $y \in \operatorname{Fix}(f)$ (the set of fixed points of $f$ ). It is known that every nonexpansive mapping having a fixed point is quasi-nonexpansive (for fixed points of nonexpansive mappings and other fixed point results see [74] and [115]).

For further results and extensions, see [58], [59], [60, [132], [133] and [70].

Amato [12, 13, 14 proposed another approach to study the connections between fixed points and completeness in metric spaces. For a metric space $(E, d)$ he considers a pair $(Y, \Psi)$, where $Y$ is a subset of $X$ and $\Psi$ is a class of mappings on $Y$. The pair $(Y, \Psi)$ is said to be a completion class for $E$ if $\Psi / \rho$ is a completion of $(E, d)$, where $\rho$ is a semimetric on $\Psi$ (defined in a concrete manner) and $\Psi / \rho$ is the quotient space with respect to the equivalence relation $f \equiv g \Longleftrightarrow \rho(f, g)=0$. Among other results, he proves that if $E$ is an infinite dimensional normed space and $K$ is a compact subset of $E$, then it is possible to take $Y=E \backslash K$ and $\Psi$ the class of all compact contractions of $Y$.

We mention also the following characterization of completeness in terms of fixed points of set-valued mappings. For a metric space $(X, \rho)$ denote by $\mathcal{P}_{c l}(X)$ the family of all nonempty closed subsets of $X$.

For a mapping $F: X \rightarrow \mathcal{P}_{c l}(X)$ consider the following two properties:

(J1) $F(F(x)) \subseteq F(x)$ for every $x \in X$;

(J2) $\forall x \in X, \forall \varepsilon>0, \exists y \in F(x)$ with $\operatorname{diam} F(y)<\varepsilon$.

For $F: X \rightarrow 2^{X}$ a point $\bar{x} \in X$ is called

- a fixed point of $F$ if $\bar{x} \in F(\bar{x})$;

- a stationary point of $F$ if $F(\bar{x})=\{\bar{x}\}$.

Theorem 1.33 (97], Corollary 1). For any metric space $(X, \rho)$ the following conditions are equivalent.

(1) The space $(X, \rho)$ is complete.

(2) Every set-valued mapping $F: X \rightarrow \mathcal{P}_{c l}(X)$ satisfying (J1) and (J2) has a fixed point.

(3) Every set-valued mapping $F: X \rightarrow \mathcal{P}_{c l}(X)$ satisfying (J1) and (J2) has a stationary point.

Characterizations of the completeness of a metric space in terms of the existence of fixed points for various classes of set-valued mappings acting on them were done by Jiang [101] and Liu [131].

We present the results from Jiang [101]. Let $(X, \rho)$ be a metric space. For a bounded subset $Y$ of $X$ denote by $\alpha(Y)$ the Kuratowski measure of noncompactness of the set $Y$ defined by

$$
\begin{aligned}
\alpha(Y):=\inf \{\varepsilon>0: & Y \text { can be covered by the union of a finite family } \\
& \text { of subsets of } X, \text { each of diameter } \leq \varepsilon\} .
\end{aligned}
$$


For a set-valued mapping $F: X \rightarrow \mathcal{P}_{c l}(X)$ one considers the following conditions:

(a) $F(F(x)) \subseteq F(x)$ for every $x \in X$;

(b) there exists a sequence $\left(x_{n}\right)$ in $X$ such that $x_{n+1} \in F\left(x_{n}\right), \forall n \in \mathbb{N}$, and $\lim _{n} \operatorname{diam}\left(F\left(x_{n}\right)\right)=0$

(c) there exists a sequence $\left(x_{n}\right)$ in $X$ such that $x_{n+1} \in F\left(x_{n}\right), \forall n \in \mathbb{N}$, and $\lim _{n} \alpha\left(F\left(x_{n}\right)\right)=0$

(d) $\lim \rho\left(x_{n}, x_{n+1}\right)=0$ for each sequence $\left(x_{n}\right)$ in $X$ such that $x_{n+1} \in F\left(x_{n}\right), \forall n \in \mathbb{N}$.

Remark 1.34. Condition (a) is identic to (J1) and it is easy to check that (J2) implies (b). Condition (d) is condition (iv) from Theorem 3.3.

One considers also the following classes of set-valued mappings $F: X \rightarrow \mathcal{P}_{c l}(X)$ :

$$
\begin{aligned}
& A B(X):=\{F: F \text { satisfies (a) and (b) }\} \\
& A C(X):=\{F: F \text { satisfies (a) and (c) }\} \\
& A D(X):=\{F: F \text { satisfies (a) and (d) }\} .
\end{aligned}
$$

Theorem 1.35 (Jiang [101, Theorems 3.1 and 3.2). For any metric space $(X, \rho)$ the following conditions are equivalent.

(1) The metric space $(X, \rho)$ is complete.

(2) Every $F$ in $A B(X)$ has a fixed point.

(3) Every $F$ in $A C(X)$ has a fixed point.

(4) Every $F$ in $A D(X)$ has a fixed point.

(5) Every $F$ in $A B(X)$ has a stationary point.

(6) Every $F$ in $A D(X)$ has a stationary point.

\section{VARIATIONAL PRINCIPLES AND COMPLETENESS}

This section is concerned with Ekeland Variational Principle (EkVP) in metric and in quasi-metric spaces and its relations to the completeness of these spaces.

2.1. Ekeland Variational Principle. The general form of EkVP is the following.

Theorem 2.1 (Ekeland Variational Principle - EkVP). Let $(X, \rho)$ be a complete metric space and $f: X \rightarrow \mathbb{R} \cup\{+\infty\}$ a lsc bounded below function. Let $\varepsilon>0$ and $x_{0} \in \operatorname{dom} f$.

Then given $\lambda>0$ there exists $z=z_{\varepsilon, \lambda} \in X$ such that

(a) $f(z)+\frac{\varepsilon}{\lambda} \rho\left(z, x_{0}\right) \leq f\left(x_{0}\right)$;

(b) $\forall x \in X, x \neq z, f(z)<f(x)+\frac{\varepsilon}{\lambda} \rho(z, x)$.

If further, $x_{0}$ satisfies the condition

$$
f\left(x_{0}\right) \leq \inf f(X)+\varepsilon,
$$

then

$$
\text { (c) } \rho\left(z, x_{0}\right) \leq \lambda \text {. }
$$

The Ekeland Variational Principle is sometimes written in the following way (see, for instance, [162] or [167, Lemma 3.13]). 
Theorem 2.2 (Ekeland Variational Principle-version b). Let $(X, \rho)$ be a complete metric space and $f: X \rightarrow \mathbb{R} \cup\{+\infty\}$ a lsc bounded below function. Let $\varepsilon>0$ and $x_{0} \in \operatorname{dom} f$.

Then given $\lambda^{\prime}>0$ there exists $z=z_{\varepsilon, \lambda^{\prime}} \in X$ such that

$$
\begin{aligned}
& \left(\mathrm{a}^{\prime}\right) \quad f(z)+\lambda^{\prime} \rho\left(z, x_{0}\right) \leq f\left(x_{0}\right) ; \\
& \left(\mathrm{b}^{\prime}\right) \quad \forall x \in X, x \neq z, \quad f(z)<f(x)+\lambda^{\prime} \rho(z, x) .
\end{aligned}
$$

If further, $x_{0}$ satisfies the condition

$$
f\left(x_{0}\right) \leq \inf f(X)+\varepsilon,
$$

then

$$
\left(\mathrm{c}^{\prime}\right) \quad \rho\left(z, x_{0}\right) \leq \frac{\varepsilon}{\lambda^{\prime}} .
$$

Proof. The equivalence between Theorem 2.1 and Theorem 2.2 follows by the substitution

$$
\lambda^{\prime}=\frac{\varepsilon}{\lambda} \Longleftrightarrow \lambda=\frac{\varepsilon}{\lambda^{\prime}}
$$

An important consequence is obtained by taking $\lambda=\sqrt{\varepsilon}$ in Theorem 2.1.

Corollary 2.3. Under the hypotheses of Theorem [2.1, for every $\varepsilon>0$ and $x_{0} \in X$ with $f\left(x_{0}\right) \leq \inf f(X)+\varepsilon$ there exists $y_{\varepsilon} \in X$ such that

$$
\begin{aligned}
& \text { (a) } f\left(y_{\varepsilon}\right)+\sqrt{\varepsilon} \rho\left(y_{\varepsilon}, x_{0}\right) \leq f\left(x_{0}\right) \text {; } \\
& \text { (b) } \forall x \in X, x \neq y_{\varepsilon}, \quad f\left(y_{\varepsilon}\right)<f(x)+\sqrt{\varepsilon} \rho\left(y_{\varepsilon}, x\right) \text {; } \\
& \text { (c) } \rho\left(y_{\varepsilon}, x_{0}\right) \leq \sqrt{\varepsilon}
\end{aligned}
$$

Taking $\lambda=1$ in Theorem 2.1, one obtains the following form of the Ekeland Variational Principle, known as the weak form of the Ekeland Variational Principle.

Corollary 2.4 (Ekeland's Variational Principle - weak form $(w E k V P))$. Let $(X, \rho)$ be a complete metric space and $f: X \rightarrow \mathbb{R} \cup\{+\infty\}$ a lsc and bounded from below proper function. Then for every $\varepsilon>0$ there exists an element $y_{\varepsilon} \in X$ such that

$$
f\left(y_{\varepsilon}\right) \leq \inf f(X)+\varepsilon,
$$

and

$$
f\left(y_{\varepsilon}\right)<f(y)+\varepsilon \rho\left(y, y_{\varepsilon}\right), \quad \forall y \in X \backslash\left\{y_{\varepsilon}\right\} .
$$

Note that the validity of Ekeland Variational Principle (in its weak form) implies the completeness of the metric space $X$. This was discovered by Weston [216] in 1977 and rediscovered by Sullivan [189] in 1981 (see also the survey [190]).

More exactly, the following result holds.

Proposition 2.5. Let $(X, \rho)$ be a metric space. If for every Lipschitz function $f: X \rightarrow$ $[0 ; \infty)$ there exists a point $z \in X$ such that

$$
f(z) \leq f(x)+\rho(z, x) \quad \text { for all } x \in X,
$$

then $X$ is complete. 
Proof. To prove the completeness of $X$, let $\left(x_{n}\right)$ be a Cauchy sequence in $X$. The inequalities $\left|\rho\left(x_{n}, x\right)-\rho\left(x_{m}, x\right)\right| \leq \rho\left(x_{n}, x_{m}\right), m, n \in \mathbb{N}$, show that $\left(\rho\left(x_{n}, x\right)\right)$ is a Cauchy sequence in $\mathbb{R}$, for every $x \in X$. Consequently, we can define a function $f: X \rightarrow \mathbb{R}$ by $f(x)=$ $2 \lim _{n \rightarrow \infty} \rho\left(x_{n}, x\right), x \in X$. The inequalities $\left|2 \rho\left(x_{n}, x\right)-2 \rho\left(x_{n}, x^{\prime}\right)\right| \leq 2 \rho\left(x, x^{\prime}\right), n \in \mathbb{N}$, yield for $n \rightarrow \infty,\left|f(x)-f\left(x^{\prime}\right)\right| \leq 2 \rho\left(x, x^{\prime}\right)$, showing that $f$ is Lipschitz. For every $\varepsilon>0$ there exists $n_{0}$ such that $2 \rho\left(x_{n}, x_{n+k}\right)<\varepsilon$, for all $n \geq n_{0}$ and all $k \in \mathbb{N}$. Letting $k \rightarrow \infty$, one obtains $0 \leq f\left(x_{n}\right) \leq \varepsilon, \forall n \geq n_{0}$, implying $\lim _{n \rightarrow \infty} f\left(x_{n}\right)=0$. By hypothesis, there exists $z \in X$ such that

$$
f(z) \leq f(x)+\rho(z, x)
$$

for all $x \in X$. Putting $x=x_{n}$ in (2.10) and letting $n \rightarrow \infty$, one obtains $f(z) \leq \frac{1}{2} f(z)$, implying $f(z)=0$, which is equivalent to $\lim _{n \rightarrow \infty} \rho\left(x_{n}, z\right)=0$, i.e., $\left(x_{n}\right)$ converges to $z$.

Remark 2.6. The validity of strong EkVP also implies the completeness of the underlying metric space $X$. Indeed, the fulfilment of (2.13).(b) implies that (2.9) holds, so that, by Proposition 2.5. the space $X$ is complete.

This also shows that the strong EkVP is equivalent to EkVP. Indeed, the proof of Theorem 2.12 shows that Theorem 2.2 implies Theorem 2.12. Since the validity of Theorem 2.12 implies the completeness of $X$ and, in its turn, this implies the validity of EkVP, the equivalence of these two principles follows.

Ekeland Variational Principle is equivalent to many important fixed point and geometric results (drop property, Caristi's fixed point theorem, the flower petal theorem, etc, see [162]). We mention here only Caristi's fixed point theorems - for both single-valued and set-valued mappings.

Theorem 2.7 (Caristi-Kirk Fixed Point Theorem). Let $(X, \rho)$ be a complete metric space and $\varphi: X \rightarrow \mathbb{R}$ a bounded from below lsc function. If the mapping $f: X \rightarrow X$ satisfies the condition

$$
\rho(x, f(x)) \leq \varphi(x)-\varphi(f(x)), x \in X,
$$

then $f$ has a fixed point in $X$.

Another consequence of EkVP is a set-valued version of Caristi's fixed point theorem.

Theorem 2.8. Let $(X, \rho)$ be a complete metric space, $\varphi: X \rightarrow \mathbb{R} \cup\{+\infty\}$ a lsc and bounded from below proper function, and $F: X \rightrightarrows X$ a set-valued mapping. If the mapping $F$ satisfies the condition

$$
\rho(x, y) \leq \varphi(x)-\varphi(y), \quad \forall x \in X, \quad \forall y \in F(x),
$$

then $F$ has a fixed point, i.e. there exists $x_{0} \in X$ such that $x_{0} \in F\left(x_{0}\right)$.

It follows that the validity of Caristi's FPT also implies the completeness of the underlying metric space.

Corollary 2.9. Let $(X, \rho)$ be a complete metric space. If every function $f: X \rightarrow X$, satisfying the hypotheses of Caristi Fixed Point Theorem for some lsc function $\varphi: X \rightarrow \mathbb{R}$, has a fixed point in $X$, then the metric space $X$ is complete.

Remark 2.10. Replacing in both Theorems 2.7 and 2.8 and in Corollary 2.9 the function $\varphi$ by $\varphi-\inf \varphi(X)$, one can consider, without restricting the generality, that the function $\varphi$ is lsc and takes values in $\mathbb{R}_{+}$. 
2.2. The strong Ekeland principle, compactness and reflexivity. Let $X$ be a Banach space and $f: X \rightarrow \mathbb{R} \cup\{\infty\}$ a function. A point $x_{0} \in \operatorname{dom}(f)$ is called

- a minimum point for $f$ if $f\left(x_{0}\right) \leq f(x)$ for all $x \in X$;

- a strict minimum point for $f$ if $f\left(x_{0}\right)<f(x)$ for all $x \in X \backslash\left\{x_{0}\right\}$;

- a strong minimum point for if $f\left(x_{0}\right)=\inf f(X)$ and every sequence $\left(x_{n}\right)$ in $X$ such that $\lim _{n} f\left(x_{n}\right)=\inf _{X} f$ is norm-convergent to $x_{0}$.

A sequence $\left(x_{n}\right)$ satisfying $\lim _{n} f\left(x_{n}\right)=\inf f(X)$ is called a minimizing sequence for $f$.

Remark 2.11. A strong minimum point is a strict minimum point, but the converse is not true.

Indeed, if there exist $z \neq z^{\prime}$ such that $f(z)=m=f\left(z^{\prime}\right)$, where $m=\inf f(X)$, then the sequence $x_{2 k-1}=z, x_{2 k}=z^{\prime}, k \in \mathbb{N}$, satisfies $\lim _{n} f\left(x_{n}\right)=m$, but it is not convergent. Also, the function $f: \mathbb{R} \rightarrow \mathbb{R}, f(x)=x^{2} e^{-x}$, has a strict minimum at $0, f(0)=0, f(n) \rightarrow$ 0 , but the sequence $(n)_{n \in \mathbb{N}}$ does not converge to 0 .

Condition (b) in Theorem 2.1 (as well as the corresponding condition in other variants) asserts, in fact, that $z$ is strict minimum point for the perturbed function $\tilde{f}:=f+\frac{\varepsilon}{\lambda} \rho(z, \cdot)$. Georgiev [71] proved a stronger variant of Ekeland variational principle, guaranteeing the existence of a strong minimum point $z$ for $\tilde{f}$. Later Turinici [211] has shown that this strong form can be deduced from Theorem 2.2 .

Theorem 2.12 (Strong Ekeland Variational Principle-amended form). Let $(X, \rho)$ be a complete metric space and $f: X \rightarrow \mathbb{R} \cup\{+\infty\}$ a lsc function bounded from below on $X$. Then for every $\gamma, \delta>0$ and $x_{0} \in \operatorname{dom}(f)$ there exists $z \in X$ such that

(a) $f(z)+\gamma \rho\left(x_{0}, z\right)<f\left(x_{0}\right)+\delta$;

(b) $f(z)<f(x)+\lambda \rho(z, x)$ for all $x \in X \backslash\{z\}$;

(c) $f\left(x_{n}\right)+\lambda \rho\left(z, x_{n}\right) \rightarrow f(z) \Rightarrow x_{n} \rightarrow z$, for every sequence $\left(x_{n}\right)$ in $X$.

Georgiev [71] deduced from Theorem 2.12 (called by him the amended strong Ekeland variational principle) the following result.

Theorem 2.13 (Strong Ekeland Variational Principle). Let $(X, \rho)$ and $f$ be as in Theorem 2.12. For $\varepsilon>0$ suppose that $x_{0} \in X$ satisfies

$$
f\left(x_{0}\right) \leq \varepsilon+\inf f(X) .
$$

Then for every $\gamma, \delta_{1}, \delta_{2}>0$ with $\gamma \delta_{1} \geq \varepsilon$, there exists $z \in X$ such that

(a) $f(z)+\gamma \rho\left(x_{0}, z\right)<f\left(x_{0}\right)+\delta_{2}$;

(b) $z$ is a strong minimum point for $\tilde{f}:=f+\gamma \rho(z, \cdot)$;

(c) $\rho\left(z, x_{0}\right) \leq \delta_{1}+\delta_{2}$.

Proof. Taking $\delta:=\delta_{2}$ in Theorem 2.12, (2.13).(a) yields (2.15).(a), which, in its turn, implies

$$
\rho\left(z, x_{0}\right) \leq \frac{1}{\gamma}\left[f\left(x_{0}\right)-f(z)\right]+\delta_{2} \leq \frac{\varepsilon}{\gamma}+\delta_{2} \leq \delta_{1}+\delta_{2} .
$$

Observe that there is a discrepancy between the conditions $\left(\mathrm{a}^{\prime}\right)$ in Theorem 2.2 and condition (a) in Theorem 2.12, condition $\left(\mathrm{a}^{\prime}\right)$ being stronger than (a). As was remarked by Suzuki [194, 200], a strong version of the Ekeland variational principle with condition 
$\left(a^{\prime}\right)$ instead of (a) can be proved by imposing supplementary conditions on the underlying metric (or normed) space $X$, which are, in some sense, also necessary.

Let $f: X \rightarrow(-\infty,+\infty]$ be a proper function defined on a metric space $(X, \rho)$. For $x_{0} \in \operatorname{dom} f$ and $\lambda>0$ consider an element $z=z_{x_{0}, \lambda}$ satisfying the following conditions:

(i) $f(z)+\lambda \rho\left(z, x_{0}\right) \leq f\left(x_{0}\right)$;

(ii) $f(z)<f(x)+\lambda \rho(z, x)$ for all $x \in X \backslash\{z\}$;

(iii) $f\left(x_{n}\right)+\lambda \rho\left(z, x_{n}\right) \rightarrow f(z) \Rightarrow x_{n} \rightarrow z$, for every sequence $\left(x_{n}\right)$ in $X$.

If $(X,\|\cdot\|)$ is a normed space, then $\rho(x, y)$ is replaced by $\|y-x\|$.

A metric space $(X, \rho)$ is called boundedly compact if every bounded closed subset of $X$ is compact. It is obvious that a boundedly compact metric space is complete, and that a normed space is boundedly compact if and only if it is finite dimensional.

Proposition 2.14. Let $(X, \rho)$ be a boundedly compact metric space, $f: X \rightarrow(-\infty,+\infty]$ a lsc bounded from below function, $x_{0} \in \operatorname{dom} f$ and $\lambda>0$.

Then there exists a point $z \in X$ satisfying the conditions (2.16).

Proof. By Theorem 2.2 there exists $z \in X$ satisfying the conditions (i) and (ii) from (2.16).

Let now $\left(x_{n}\right)$ be a sequence in $X$ such that $\lim _{n}\left[f\left(x_{n}\right)+\lambda \rho\left(z, x_{n}\right)\right]=f(z)$ and suppose, by contradiction, that $\left(x_{n}\right)$ does not converge to $z$. Then there exist $\gamma>0$ and a subsequence $y_{k}=x_{n_{k}}, k \in \mathbb{N}$, of $\left(x_{n}\right)$ such that $\rho\left(y_{k}, z\right) \geq \gamma$ for all $k \in \mathbb{N}$.

Let $k_{0} \in \mathbb{N}$ be such that $f\left(y_{k}\right)+\lambda \rho\left(z, y_{k}\right) \leq f(z)+1$ for all $k>k_{0}$. The inequalities

$$
\begin{aligned}
\lambda \rho\left(z, y_{k}\right) & =f\left(y_{k}\right)+\lambda \rho\left(z, y_{k}\right)-f\left(y_{k}\right) \\
& \leq f(z)+1-\inf f(X),
\end{aligned}
$$

valid for all $k>k_{0}$, show that the sequence $\left(y_{k}\right)$ is bounded. Consequently, it contains a subsequence $\left(y_{k_{i}}\right)$ converging to some $y \in X \backslash\{z\}$. Taking into account the lsc of the function $f$, one obtains

$$
f(y)+\lambda \rho(z, y) \leq \liminf _{i}\left[f\left(y_{k_{i}}\right)+\lambda \rho\left(z, y_{k_{i}}\right)\right]=f(z),
$$

in contradiction to (2.16) . (ii).

Remark 2.15. 1. Let $X$ be a vector space. A function $f: X \rightarrow \mathbb{R} \cup\{\infty\}$ is called quasi-convex if

$$
f((1-t) x+t y) \leq \max \{f(x), f(y)\},
$$

for all $x, y \in X$ and $t \in[0,1]$. This is equivalent to the fact that the sublevel sets $\{x \in X$ : $f(x) \leq \alpha\}$ are convex for all $\alpha \in \mathbb{R}$ (see [148]).

2. One says that a Banach space $X$ is a dual Banach space if there exists a Banach space $Y$ such that $Y^{*}=X$. Obviously, a reflexive Banach space is a dual Banach space with $X=\left(X^{*}\right)^{*}$ and, in this case, the weak topology and the weak* topology on $X$ agree.

In the Banach space case the following results can be proved.

Proposition 2.16 ([194]). Let $X$ be a Banach space, $f: X \rightarrow(-\infty,+\infty]$ a bounded from below function, $x_{0} \in \operatorname{dom} f$ and $\lambda>0$.

1. If $X$ is a dual Banach space and $f$ is $w^{*}$-lsc, then there exists a point $z \in X$ satisfying (2.16) with $x_{n} \stackrel{w^{*}}{\longrightarrow} x$ in the condition (iii).

2. Suppose that the Banach space $X$ is reflexive. If $f$ is weakly lsc, then there exists a point $z \in X$ satisfying the conditions (2.16). The same is true if $f$ is quasi-convex and norm-lsc. 
Proof. 1. Since

$$
x_{n} \stackrel{\|\cdot\|}{\longrightarrow} x \Rightarrow x_{n} \stackrel{w^{*}}{\longrightarrow} x \Rightarrow f(x) \leq \liminf _{n} f\left(x_{n}\right),
$$

it follows that $f$ is also lsc on $(X,\|\cdot\|)$. By Theorem 2.2, there exists $z \in X$ satisfying the conditions (i) and (ii) from (2.16).

Let $\left(x_{n}\right)$ be a sequence in $X$ such that

$$
\lim _{n \rightarrow \infty}\left[f\left(x_{n}\right)+\lambda\left\|x_{n}-z\right\|\right]=f(z) .
$$

The inequalities

$$
\begin{aligned}
\left\|x_{n}\right\| & \leq\|z\|+\left\|x_{n}-z\right\| \\
& \leq\|z\|+\frac{f\left(x_{n}\right)+\lambda\left\|x_{n}-z\right\|}{\lambda}-\frac{1}{\lambda} \inf f(X)
\end{aligned}
$$

show that the sequence $\left(x_{n}\right)$ is norm-bounded. If $\left(x_{n}\right)$ is not $w^{*}$-convergent to $z$, then, by the Alaoglu-Bourbaki theorem, there exist a subnet $\left(y_{i}: i \in I\right)$ of the sequence $\left(x_{n}\right)$ and $y \neq z$ in $X$ such that $y_{i} \stackrel{w^{*}}{\longrightarrow} y$. Since the norm $\|\cdot\|$ is also $w^{*}$-lsc we have

$$
f(y)+\lambda\|y-z\| \leq \liminf _{i}\left[f\left(y_{i}\right)+\lambda\left\|y_{i}-z\right\|\right]=f(z),
$$

in contradiction to (2.16).(ii).

2. Since a reflexive Banach space $X$ is a dual Banach space and the weak and weak* topologies agree on $X$, Theorem 2.2 and the first statement of the proposition show that, in both cases, there exists $z \in X$ satisfying the conditions (i) and (ii) from (2.16).

Let $\left(x_{n}\right)$ be a sequence in $X$ such that

$$
\lim _{n}\left[f\left(x_{n}\right)+\lambda\left\|z-x_{n}\right\|\right]=f(z) .
$$

I. The sequence $\left(x_{n}\right)$ converges weakly to $z$.

If $f$ is weakly lsc, then I follows from statement 1 of the proposition.

Suppose that $f$ is quasi-convex and norm-lsc. The function $f$ is quasi-convex if and only if the set $\{x \in X: f(x) \leq \alpha\}$ is convex for every $\alpha \in \mathbb{R}$, and lsc if and only if the set $\{x \in X: f(x) \leq \alpha\}$ is closed for every $\alpha \in \mathbb{R}$. Since norm and weak closed convex sets are the same, it follows that $f$ is also weakly lsc. Hence, by the first part of the proposition the sequence $\left(x_{n}\right)$ converges weakly to $z$.

II. The sequence $\left(x_{n}\right)$ converges strongly to $z$.

Suppose again that the sequence $\left(x_{n}\right)$ does not converge strongly to $z$. Then there exists $\varepsilon>0$ and a subsequence $\left(x_{n_{k}}\right)$ of $\left(x_{n}\right)$ such that

$$
\left\|z-x_{n_{k}}\right\|>2 \varepsilon \text { and } f\left(x_{n_{k}}\right)+\lambda\left\|z-x_{n_{k}}\right\|<f(z)+\lambda \varepsilon
$$

for all $k \in \mathbb{N}$.

The inequalities

$$
f\left(x_{n_{k}}\right)+2 \lambda \varepsilon<f\left(x_{n_{k}}\right)+\lambda\left\|z-x_{n_{k}}\right\|<f(z)+\lambda \varepsilon,
$$

yield

$$
f\left(x_{n_{k}}\right)<f(z)-\lambda \varepsilon
$$

for all $k \in \mathbb{N}$.

Since, by I, $\left(x_{n_{k}}\right)$ converges weakly to $z$ and $f$ is weakly lsc, the above inequalities lead to the contradiction

$$
f(z) \leq \liminf _{k} f\left(x_{n_{k}}\right) \leq f(z)-\lambda \varepsilon .
$$


As it was shown by Suzuki [200, in some sense, the results from Propositions 2.14 and 2.16] are the best that can be expected.

Theorem 2.17. For a metric space $(X, \rho)$ the following are equivalent.

1. The metric space $X$ is boundedly compact.

2. For every proper lsc bounded from below function $f: X \rightarrow(-\infty,+\infty], x_{0} \in \operatorname{dom} f$ and $\lambda>0$ there exists a point $z \in X$ satisfying the conditions (2.16).

3. For every Lipschitz function $f: X \rightarrow[0,+\infty), x_{0} \in \operatorname{dom} f$ and $\lambda>0$ there exists a point $z \in X$ satisfying the conditions (2.16).

Proof. The implication $1 \Rightarrow 2$ was proved in Proposition 2.14, while the implication $2 \Rightarrow$ 3 is obvious.

It remains to prove the implication $3 \Rightarrow 1$.

Observe that, by Proposition 2.5, the metric space $X$ is complete. Suppose that it is not boundedly compact. Then there exists a closed bounded subset $Y$ of $X$ which is not compact. Since $X$ is complete it follows that $Y$ is not totally bounded. Consequently there exist $\varepsilon>0$ and a subset $\left\{y_{n}: n \in \mathbb{N}\right\}$ of $Y$ such that

$$
\rho\left(y_{n}, y_{m}\right)>3 \varepsilon \text { for all } m \neq n \text {. }
$$

Let

$$
\delta=\sup _{m} \rho\left(y_{1}, y_{m}\right) \quad \text { and } \quad B_{n}=B\left[y_{n}, \varepsilon\right], n \in \mathbb{N} .
$$

It follows

$$
\delta>3 \varepsilon, \quad 1-\frac{\rho\left(y_{1}, y_{n}\right)}{\delta} \geq 0 \quad \text { and } \quad B_{n} \cap B_{m}=\emptyset,
$$

for all $n$ and all $m \neq n$.

For $n \in \mathbb{N}$ put

$$
f_{n}(x):=1-\frac{\rho\left(y_{1}, y_{n}\right)}{\delta}+\frac{1}{n}+\frac{\rho\left(x, y_{n}\right)}{\varepsilon}, x \in X .
$$

It follows that the function $f_{n}$ is $\frac{1}{\varepsilon}$-Lipschitz on $X$.

Defining $f: X \rightarrow[0 ; \infty)$ by

$$
f(x):=\min \left\{1, \inf _{n} f_{n}(x)\right\}, x \in X,
$$

the function $f$ will be $\frac{1}{\varepsilon}$-Lipschitz on $X$ too.

We intend to show that

$$
f(x)= \begin{cases}\min \left\{1, f_{n}(x)\right\} & \text { if } \quad x \in B_{n} \text { for some } n \geq 2, \\ 1 & \text { otherwise. }\end{cases}
$$

Let $x \in B_{n}$ for some $n \geq 2$. If $m \neq n$ then

$$
f_{m}(x)=1-\frac{\rho\left(y_{1}, y_{m}\right)}{\delta}+\frac{1}{m}+\frac{\rho\left(x, y_{m}\right)}{\varepsilon}>2+\frac{1}{m},
$$

because $\rho\left(x, y_{m}\right) \geq \rho\left(y_{n}, y_{m}\right)-\rho\left(y_{n}, x\right)>2 \varepsilon$.

Consequently

$$
f(x)=\min \left\{1, \inf _{n} f_{n}(x)\right\}=\min \left\{1, f_{n}(x), \inf _{m \neq n} f_{m}(x)\right\}=\min \left\{1, f_{n}(x)\right\} .
$$


Let $x \in B_{1}$. Then

$$
f_{1}(x)=2+\frac{\rho\left(x, y_{1}\right)}{\varepsilon} \geq 2 \quad \text { and } \quad f_{m}(x)>2+\frac{1}{m} \quad \text { for } m \geq 2,
$$

implying $f(x)=1$. In particular $f\left(y_{1}\right)=1$.

Let now $x \in X \backslash \bigcup_{k} B_{k}$. Then for every $n \in \mathbb{N}$,

$$
f_{n}(x)=1-\frac{\rho\left(y_{1}, y_{n}\right)}{\delta}+\frac{1}{n}+\frac{\rho\left(x, y_{n}\right)}{\varepsilon}>1+\frac{1}{n},
$$

because $\rho\left(x, y_{n}\right)>\varepsilon$. Consequently $f(x)=1$ and (2.17) holds.

Let $x_{0}=y_{1}$ and $\lambda=\delta^{-1}$. We show that if $z \in X$ satisfies the conditions (i) and (ii) from (2.16), then the condition (iii) is not satisfied.

We show first that $z=y_{1}$. If $f(z)<1$, then $z \in B_{n}$ for some $n \geq 2$, implying

$$
\begin{aligned}
f_{n}(z)+\frac{\rho\left(z, y_{1}\right)}{\delta}=1 & +\frac{1}{n}-\frac{\rho\left(y_{1}, y_{n}\right)}{\delta}+\frac{\rho\left(z, y_{1}\right)}{\delta}+\frac{\rho\left(z, y_{n}\right)}{\varepsilon} \\
& \geq 1+\frac{1}{n}-\frac{\rho\left(z, y_{n}\right)}{\delta}+\frac{\rho\left(z, y_{n}\right)}{\varepsilon} \geq 1+\frac{1}{n}>1=f\left(y_{1}\right),
\end{aligned}
$$

in contradiction to (2.16). (i).

Hence $f(z)=1$ and, by (2.16). (i),

$$
1+\frac{\rho\left(z, y_{1}\right)}{\delta} \leq f\left(y_{1}\right)=1
$$

which implies $\rho\left(z, y_{1}\right)=0$ and $z=y_{1}$.

Consider now the sequence $\left(y_{n}\right)$ in $X$. Then, by (2.17),

$$
\alpha_{n}:=f\left(y_{n}\right)+\frac{\rho\left(y_{1}, y_{n}\right)}{\delta}=\min \left\{1,1+\frac{1}{n}-\frac{\rho\left(y_{1}, y_{n}\right)}{\delta}\right\}+\frac{\rho\left(y_{1}, y_{n}\right)}{\delta},
$$

for every $n \geq 2$.

If

$$
1+\frac{1}{n}-\frac{\rho\left(y_{1}, y_{n}\right)}{\delta}>1
$$

then

$$
\alpha_{n}=1+\frac{\rho\left(y_{1}, y_{n}\right)}{\delta}<1+\frac{1}{n}
$$

and

$$
\alpha_{n}=1+\frac{1}{n}
$$

if

$$
1+\frac{1}{n}-\frac{\rho\left(y_{1}, y_{n}\right)}{\delta}<1
$$

Taking into account (2.16).(ii) and these relations, one obtains

$$
1=f\left(y_{1}\right)<\alpha_{n} \leq 1+\frac{1}{n}
$$

for all $n \geq 2$. It follows $\lim _{n \rightarrow \infty} \alpha_{n}=1$, but the sequence $\left(y_{n}\right)$ does not converge.

A similar result holds in the case of normed spaces.

Theorem 2.18. For a normed space $(X,\|\cdot\|)$ the following are equivalent.

1. $X$ is a reflexive Banach space.

2. For every proper lsc bounded from below quasi-convex function $f: X \rightarrow(-\infty,+\infty]$, $x_{0} \in \operatorname{dom} f$ and $\lambda>0$ there exists a point $z \in X$ satisfying the conditions (2.16). 
3. For every Lipschitz convex function $f: X \rightarrow[0,+\infty), \quad x_{0} \in \operatorname{dom} f$ and $\lambda>0$ there exists a point $z \in X$ satisfying the conditions (2.16).

Proof. The implication $1 \Rightarrow 2$ was proved in Proposition 2.16 , while the implication $2 \Rightarrow$ 3 is obvious.

It remains to prove the implication $3 \Rightarrow 1$.

Observe that, by Proposition 2.5, the normed space $X$ is complete. By James' theorem on the characterization of reflexivity, the space $X$ will be reflexive if every $x^{*} \in S_{X^{*}}$ (the unit sphere of the dual space $X^{*}$ ) attains its norm on $S_{X}$ (the unit sphere of the space $X)$.

For $x^{*} \in S_{X^{*}}$ consider the convex 1-Lipschitz function $f(x):=\left|x^{*}(x)-1\right|, x \in X$. Then for $x_{0}=0$ and $\lambda=1$ there exists $z \in X$ satisfying the conditions (2.16).

Suppose that $z=0$. Let $\left(x_{n}\right)$ be sequence in $S_{X}$ such that $\lim _{n \rightarrow \infty} x^{*}\left(x_{n}\right)=\left\|x^{*}\right\|=1$. Then

$$
\lim _{n \rightarrow \infty}\left[f\left(x_{n}\right)+\left\|0-x_{n}\right\|\right]=\lim _{n \rightarrow \infty}\left[\left|x^{*}\left(x_{n}\right)-1\right|+1\right]=1=f(0),
$$

but the sequence $\left(x_{n}\right)$ does not converge to 0 , in contradiction to (2.16).(iii).

Consequently $z \neq 0$. Taking into account (2.16).(i), one obtains

$$
\left|x^{*}(z)-1\right|+\|z\| \leq f(0)=1 \Longrightarrow\|z\| \leq 1-\left|x^{*}(z)-1\right| \leq\left|x^{*}(z)\right| .
$$

Since $\left|x^{*}(z)\right| \leq\|z\|$ it follows $\left|x^{*}(z)\right|=\|z\|$. This last equality implies $\|z\|=1$, so that $x^{*}$ attains its norm at $z \in S_{X}$. The reflexivity of $X$ is proved.

It is known that every lsc function attains its minimum on a compact space, a property which is actually equivalent to compactness.

Theorem 2.19 (200]). For a metric space $(X, \rho)$ the following are equivalent.

1. The metric space $X$ is compact.

2. Every lsc function $f: X \rightarrow \mathbb{R} \cup\{\infty\}$ attains its minimum on $X$.

3. Every Lipschitz function $f: X \rightarrow \mathbb{R}$ attains its minimum on $X$.

Proof. The implication $1 \Rightarrow 2$ is well known, but, for convenience, we include the simple proof. Suppose that $f$ is not identically equal to $+\infty$ and let $m=\inf f(X)$ and let $\left(x_{n}\right)$ be a sequence in $X$ such that $f\left(x_{n}\right) \rightarrow m$. By the compactness of $X$ there exist a subsequence $\left(x_{n_{k}}\right)$ of $\left(x_{n}\right)$ and $x \in X$ such that $x_{n_{k}} \rightarrow x$ as $k \rightarrow \infty$. But then

$$
m \leq f(x) \leq \liminf _{k} f\left(x_{n_{k}}\right)=m,
$$

implying $f(x)=m \in \mathbb{R}$.

The implication $2 \Rightarrow 3$ is obvious.

$3 \Rightarrow 1$. The compactness of $X$ is equivalent to its completeness and total boundedness.

$X$ is complete.

Let $\left(x_{n}\right)$ be a Cauchy sequence in $X$. Then, as in the proof of Proposition 2.5, the function $f(x)=\lim _{n} \rho\left(x_{n}, x\right), x \in X$, is well defined, 1- Lipschitz and inf $f(X)=0$. By hypothesis, there exists $x \in X$ such that $0=f(x)=\lim _{n} \rho\left(x_{n}, x\right)$, showing that the sequence $\left(x_{n}\right)$ converges to $x$.

$X$ is totally bounded.

If contrary, then there exist $\varepsilon>0$ and a sequence $\left(x_{n}\right)$ in $X$ such that

$$
\rho\left(x_{n}, x_{m}\right) \geq \varepsilon,
$$


for all $m \neq n$ in $\mathbb{N}$. Let $Y=\left\{x_{n}: n \in \mathbb{N}\right\} \subseteq X$ and $g: Y \rightarrow \mathbb{R}$ be defined by $g\left(x_{n}\right)=$ $1 / n, n \in \mathbb{N}$. Then, by (2.18),

$$
\left|g\left(x_{n}\right)-g\left(x_{m}\right)\right|=\left|\frac{1}{n}-\frac{1}{m}\right| \leq 1 \leq \frac{1}{\varepsilon} \rho\left(x_{n}, x_{m}\right),
$$

for all $m, n \in \mathbb{N}$, that is, $g$ is $1 / \varepsilon$-Lipschitz. The function $f: X \rightarrow \mathbb{R}$ given by

$$
f(x)=\inf _{n}\left[g\left(x_{n}\right)+K \rho\left(x_{n}, x\right)\right], x \in X,
$$

where $K=1 / \varepsilon$, is a $K$-Lipschitz extension of $g$ (see, for instance, [51, Th. 4.1.1]). We have

$$
0 \leq \inf f(X) \leq \inf _{n} g\left(x_{n}\right)=0,
$$

so that inf $f(X)=0$. If $f(x)=0$ for some $x \in X$, then, by the definition of $f$, there exists a subsequence $\left(x_{n_{k}}\right)$ of $\left(x_{n}\right)$ such that

$$
\lim _{k}\left[\frac{1}{n_{k}}+K \rho\left(x_{n_{k}}, x\right)\right]=0
$$

implying $\lim _{k} x_{n_{k}}=x$. But this is impossible, because, by (2.18), the sequence $\left(x_{n}\right)$ has no Cauchy subsequences.

Remark 2.20. The proof given here is different from that in [200].

\section{OTHER PRINCIPLES}

In this subsection we shall present some results equivalent to Ekeland Variational Principle.

3.1. Takahashi minimization principle. The first one is that of Takahashi [203] (see also [102] and [205, T. 2.1.1]).

Theorem 3.1 (Takahashi Principle). Let $(X, \rho)$ be a complete metric space and $f: X \rightarrow$ $\mathbb{R} \cup\{\infty\}$ a lsc bounded from below proper function. If the following condition

$$
\forall x \in X, \inf f(X)<f(x) \Rightarrow \exists y_{x} \in X \backslash\{x\}, f\left(y_{x}\right)+\rho\left(x, y_{x}\right) \leq f(x),
$$

holds, then there exists $x_{0} \in X$ such that $f\left(x_{0}\right)=\inf f(X)$.

Proof. In fact, Takahashi's theorem is an immediate consequence of EkVP. Indeed, by Theorem 2.1, given $\varepsilon=\lambda=1$, there exists $z \in X$ such that

$$
f(z)<f(x)+\rho(x, z),
$$

for all $x \in X \backslash\{z\}$. If $f(z)>\inf f(X)$, then, by (3.1), there exists $y \in X \backslash\{z\}$ such that

$$
f(y)+\rho(z, y) \leq f(z),
$$

in contradiction to (3.2), so that $f(z)=\inf f(X)$.

The validity of Takahashi's minimum principle also implies the completeness of the underlying metric space.

Proposition 3.2. Let $(X, \rho)$ be a metric space. If every bounded below Lipschitz function $f: X \rightarrow \mathbb{R}$ satisfying the condition (3.1) attains its minimum on $X$, then the metric space $X$ is complete. 
Proof. We proceed by contradiction: suppose that the metric space $X$ is not complete and show that there exists a Lipschitz function $f: X \rightarrow \mathbb{R}$ satisfying (3.1) and which does not attain its minimum on $X$.

If $X$ is not complete, then $X$ contains a Cauchy sequence $\left(x_{n}\right)$ which has no limit. We shall use the function

$$
f(x)=2 \lim _{n \rightarrow \infty} \rho\left(x, x_{n}\right), \quad x \in X,
$$

similar to that considered in the proof of Proposition 2.5. The function $f$ is 2-Lipschitz and $f(x)>0$ for all $x \in X$. Indeed, if $f(x)=0$ for some $x \in X$, then $\lim _{n \rightarrow \infty} \rho\left(x, x_{n}\right)=0$, i.e. the sequence $\left(x_{n}\right)$ would converge to $x$, in contradiction to the hypothesis.

As it was shown in the proof of Proposition 2.5.

$$
\lim _{n \rightarrow \infty} f\left(x_{n}\right)=0 .
$$

Consequently

$$
0 \leq \inf f(X) \leq \inf \left\{f\left(x_{n}\right): n \in \mathbb{N}\right\}=0,
$$

so that inf $f(X)=0$. Since $f(x)>0$ for all $x \in X$, it follows that $f$ does not attains its minimum value on $X$.

It remains to show that $f$ satisfies (3.1). If $x \in X$, then $f(x)>0=\inf f(X)$.

As $\lim _{n} \rho\left(x, x_{n}\right)=2^{-1} f(x)$, there exists $n_{1} \in \mathbb{N}$ such that

$$
\rho\left(x, x_{n}\right) \leq \frac{2}{3} \cdot f(x)
$$

for all $n \geq n_{1}$.

Since the sequence $\left(x_{n}\right)$ is Cauchy, there exists $n_{2} \in \mathbb{N}$ such that

$$
\rho\left(x_{n}, x_{n+k}\right) \leq \frac{1}{6} \cdot f(x),
$$

for all $n \geq n_{2}$ and all $k \in \mathbb{N}$. Keeping $n \geq n_{2}$ fixed and letting $k \rightarrow \infty$, one obtains

$$
f\left(x_{n}\right) \leq \frac{1}{3} \cdot f(x)
$$

for all $n \geq n_{2}$. Then, taking $n_{0} \geq \max \left\{n_{1}, n_{2}\right\}$ such that $x_{n_{0}} \neq x$ (such an $n_{0}$ exists because the sequence $\left(x_{n}\right)$ is not convergent), the inequalities (3.3) and (3.4) imply

$$
\rho\left(x, x_{n_{0}}\right)+f\left(x_{n_{0}}\right) \leq \frac{5}{6} f(x)<f(x),
$$

i.e. (3.1) is satisfied by $y_{x}=x_{n_{0}}$.

3.2. Dancs-Hegedüs-Medvegyev principle. Another result, also equivalent to Ekeland Variational Principle, was proved by Dancs, Hegedüs and Medvegyev [53].

Theorem 3.3. Let $(X, \rho)$ be a complete metric space and $F: X \rightrightarrows X$ a set-valued function satisfying the conditions:

(i) $F(x)$ is closed for every $x \in X$;

(ii) $x \in F(x)$ for every $x \in X$;

(iii) $x_{2} \in F\left(x_{1}\right) \Rightarrow F\left(x_{2}\right) \subseteq F\left(x_{1}\right)$ for all $x_{1}, x_{2} \in X$;

(iv) $\lim _{n} \rho\left(x_{n}, x_{n+1}\right)=0$ for every sequence $\left(x_{n}\right)$ in $X$ such that $x_{n+1} \in F\left(x_{n}\right), \forall n \in \mathbb{N}$.

Then there exists $x_{0} \in X$ such that $F\left(x_{0}\right)=\left\{x_{0}\right\}$. Moreover, for every $\bar{x} \in X$, there exists such a point in $F(\bar{x})$. 
This result admits an equivalent formulation in terms of an order on $X$.

Theorem 3.4. Let $(X, \rho)$ be a complete metric space and $\preceq$ a closed partial order on $X$. If $\lim _{n} \rho\left(x_{n}, x_{n+1}\right)=0$ for every increasing sequence $x_{1} \preceq x_{2} \preceq \ldots$ in $X$, then there is a maximal element in $X$. In fact, for every $x \in X$ the set $\{y \in X: x \preceq y\}$ contains a maximal element of $(X, \preceq)$.

Proof. The equivalence is proved by considering the sets $\Phi(x)=\{y \in X: x \preceq y\}$.

An order $\preceq$ on a metric space is said to be closed if $x_{n} \preceq y_{n}$, for all $n \in \mathbb{N}$, implies $\lim _{n} x_{n} \preceq \lim _{n} y_{n}$, provided both limits exist. This is equivalent to the fact that the graph of $\preceq, \operatorname{Graph}(\preceq):=\{(x, y) \in X \times X: x \preceq y\}$ is closed in $X \times X$ with respect to the product topology.

Remark 3.5. If $F: X \rightrightarrows X$ is a set-valued mapping, then for every $x_{0} \in X$, a sequence $\left(x_{n}\right)$ satisfying $x_{n} \in F\left(x_{n-1}\right), n \in \mathbb{N}$, is called a generalized Picard sequence. For the properties of set-valued Picard operators, defined in terms of the convergence of generalized Picard sequences, see the surveys [164, 165].

Remark 3.6. As it is remarked in [53, Th. 3.3], the converse completeness property holds in this case too, i.e. the validity of Theorem 3.3 for every set-valued mapping $F$ on an arbitrary metric space $X$ implies the completeness of $X$.

3.3. Arutyunov's principle. A result similar to Takahashi's minimization principle (Theorem 3.1), under a slightly relaxed condition on the function $f$, was found by Arutyunov and Gel'man [23] and Arutyunov [22]. For recent further developments see [24] and [25].

Let $(X, \rho)$ be a metric space and $f: X \rightarrow[0,+\infty]$ a function. One considers the following condition on $f$ :

$$
x_{n} \rightarrow \bar{x} \text { and } f\left(x_{n}\right) \rightarrow 0 \Rightarrow f(\bar{x})=0,
$$

for any sequence $\left(x_{n}\right)$ in $X$ and any $\bar{x} \in X$.

Remark 3.7. It is obvious that any lsc or with closed graph bounded below proper function $f: X \rightarrow[0,+\infty]$ satisfies (3.5).

The case when $f$ has closed graph is clear. If $f$ is lsc, then for any sequence $\left(x_{n}\right)$ as in (3.5),

$$
0 \leq f(\bar{x}) \leq \liminf _{n \rightarrow \infty} f\left(x_{n}\right)=0 .
$$

Theorem 3.8 ([23]). Let $(X, \rho)$ be a complete metric space and $f: X \rightarrow[0,+\infty]$ a function satisfying the condition (3.5). Suppose that there exist $k_{1}>0$ and $0<k_{2}<1$ such that for every $x \in X$ with $f(x)>0$ there exists $x^{\prime} \in X \backslash\{x\}$ satisfying

$$
\text { (i) } \rho\left(x, x^{\prime}\right) \leq k_{1} f(x) \text { and (ii) } f\left(x^{\prime}\right) \leq k_{2} f(x) \text {. }
$$

Then for every $x_{0} \in \operatorname{dom} f$ there exists $\bar{x} \in X$ such that

$$
\text { (a) } \quad f(\bar{x})=0=\inf f(X) \quad \text { and } \quad\left(\text { b) } \quad \rho\left(x_{0}, \bar{x}\right) \leq \frac{k_{1}}{1-k_{2}} f\left(x_{0}\right)\right. \text {. }
$$

We shall give a proof of an extension of this theorem given by Arutyunov [22].

Theorem $3.9([22)$. Let $(X, \rho)$ be a complete metric space and $f: X \rightarrow[0,+\infty] a$ function satisfying (3.5). Suppose that there exist a usc function $\varphi: \mathbb{R}_{+} \rightarrow \mathbb{R}_{+}$such that

$$
\varphi(t)<t \text { for every } t>0 \text {, }
$$


and $\gamma>0$ such that for every $x \in X$ with $f(x)>0$ there exists $x^{\prime} \in X \backslash\{x\}$ satisfying

$$
\text { (i) } f\left(x^{\prime}\right)+\gamma \rho\left(x, x^{\prime}\right) \leq f(x) \text { and (ii) } f\left(x^{\prime}\right) \leq \varphi(f(x)) \text {. }
$$

Then for every $x_{0} \in \operatorname{dom} f$ there exists $\bar{x} \in X$ such that

$$
\text { (a) } \quad f(\bar{x})=0=\inf f(X) \quad \text { and } \quad \text { (b) } \quad \rho\left(x_{0}, \bar{x}\right) \leq \frac{1}{\gamma} f\left(x_{0}\right) \text {. }
$$

Remark 3.10. Replacing the metric $\rho$ with the equivalent one $\tilde{\rho}=\gamma \rho$, the condition (3.9).(i) becomes Takahashi's condition (3.1). Arutyunov [22] calls it a Caristi type condition.

Let us show first that Theorem 3.9 implies Theorem 3.8. Let $x \in X$. Then there exists $x^{\prime} \neq x$ in $X$ satisfying (3.6). But (3.6). (ii) means that $f\left(x^{\prime}\right) \leq \varphi(f(x))$, where $\varphi(t)=k_{2} t, t \in \mathbb{R}_{+}$. Also, multiplying the first inequality in (3.6) by $1-k_{2}$, the second by $k_{1}$, adding them and dividing the result by $k_{1}$, one obtains

$$
f\left(x^{\prime}\right)+\frac{1-k_{2}}{k_{1}} \rho\left(x, x^{\prime}\right) \leq f(x),
$$

i.e., (3.9) .(i) holds with $\gamma=\left(1-k_{2}\right) / k_{1}$.

Proof of Theorem [3.9. It is obvious that $\inf f(X)=0$.

Indeed, $f(x) \geq 0$ for all $x \in X$ and, by (3.9) .(i), for every $x \in X$ with $f(x)>0$ there exists $x^{\prime} \in X$ with $f\left(x^{\prime}\right)<f(x)$.

For $x_{0} \in \operatorname{dom} f$ consider a sequence $\left(x_{k}\right)_{k=0}^{\infty}$ satisfying

$$
\text { (i) } f\left(x_{k+1}\right)+\gamma \rho\left(x_{k+1}, x_{k}\right) \leq f\left(x_{k}\right) \quad \text { and } \quad \text { (ii) } \quad f\left(x_{k+1}\right) \leq \varphi\left(f\left(x_{k}\right)\right) \text {, }
$$

for all $k=0,1, \ldots$. This is possible by (3.9). Indeed, if $x_{0}, \ldots, x_{n}$ satisfy (3.11) for $k=0, \ldots, n-1$, then, by (3.9), there exists $x_{n+1} \neq x_{n}$ such that

$$
\text { (i) } f\left(x_{n+1}\right)+\gamma \rho\left(x_{n+1}, x_{n}\right) \leq f\left(x_{n}\right) \quad \text { and } \quad \text { (ii) } \quad f\left(x_{n+1}\right) \leq \varphi\left(f\left(x_{n}\right)\right) \text {. }
$$

By (3.11).(i), $f\left(x_{k+1}\right)<f\left(x_{k}\right), k \in \mathbb{N}_{0}$, so there exists $\alpha:=\lim _{k \rightarrow \infty} f\left(x_{k}\right) \geq 0$. By the same inequality,

$$
\begin{aligned}
\gamma \rho\left(x_{n}, x_{n+k}\right) & \leq \gamma \rho\left(x_{n}, x_{n+1}\right)+\cdots+\gamma \rho\left(x_{n+k-1}, x_{n+k}\right) \\
& \leq f\left(x_{n}\right)-f\left(x_{n+k}\right),
\end{aligned}
$$

for all $n \in \mathbb{N}_{0}$ and $k \in \mathbb{N}$.

By the convergence of the sequence $\left(f\left(x_{n}\right)\right)$ this implies that the sequence $\left(x_{n}\right)$ is Cauchy, so, by the completeness of the metric space $X$, it converges to some $\bar{x} \in X$. By (3.11).(ii) and the usc of the function $\varphi$,

$$
0 \leq \alpha=\lim _{k \rightarrow \infty} f\left(x_{k+1}\right) \leq \limsup _{k \rightarrow \infty} \varphi\left(f\left(x_{k}\right)\right) \leq \varphi(\alpha) .
$$

By (3.8), this implies $\alpha=0$, so that, by(3.5), $f(\bar{x})=0=\inf f(X)$.

Let us prove now (3.10) $)$ (b). By (3.12) for $n=0$

$$
\gamma \rho\left(x_{0}, x_{k}\right) \leq f\left(x_{0}\right)-f\left(x_{k}\right),
$$

for all $k \in \mathbb{N}$.

Letting $k \rightarrow \infty$, one obtains $\gamma \rho\left(x_{0}, \bar{x}\right) \leq f\left(x_{0}\right)$.

Remark 3.11. If the function $f$ is lsc and satisfies the condition (3.9).(i), then the conclusions of Theorem 3.9 can be obtained directly from Ekeland's Variational Principle (Theorem 2.1). As it is noticed in [22], this remark is due to B. D. Gel'man. 
As above, we have inf $f(X)=0$, so that there is nothing to prove if $f\left(x_{0}\right)=0$.

Suppose $f\left(x_{0}\right)>0$ and put $\varepsilon:=f\left(x_{0}\right)$ and $\lambda:=\varepsilon / \gamma$. Then $\varepsilon / \lambda=\gamma$, so that, by Theorem 2.1, there exists $\bar{x} \in X$ such that

$$
\begin{aligned}
& \text { (a) } \quad \forall x \in X, x \neq \bar{x}, \quad f(\bar{x})<f(x)+\gamma \rho(\bar{x}, x) \text {; } \\
& \text { (b) } \rho\left(\bar{x}, x_{0}\right) \leq \lambda=\frac{\varepsilon}{\gamma}=\frac{1}{\gamma} f\left(x_{0}\right) .
\end{aligned}
$$

Since (3.13) .(b) agrees with (3.10) .(b), we have only to show that $f(\bar{x})=0$. If $f(\bar{x})>0$, then, by (3.9).(i), there exists $x^{\prime} \neq \bar{x}$ in $X$ such that

$$
f\left(x^{\prime}\right)+\gamma \rho\left(\bar{x}, x^{\prime}\right) \leq f(\bar{x}),
$$

in contradiction to (3.13) $)$ (a).

3.4. Weak sharp minima and completeness. Let $(X, \rho)$ be a metric space and $f$ : $X \rightarrow \mathbb{R} \cup\{\infty\}$ bounded below proper function with

$$
\mu:=\inf f(X)>-\infty .
$$

For $\alpha \in \mathbb{R}$ let

$$
S_{\alpha}(f)=\{x \in X: f(x) \leq \alpha\} .
$$

It follows that

$$
S_{\mu}(f)=\{x \in X: f(x)=\mu\}
$$

and

$$
S_{\alpha}(f) \subseteq S_{\beta}(f) \text { for } \alpha \leq \beta .
$$

One says that $f$ has global weak sharp minima with constant $\lambda>0$ if $S_{\mu} \neq \emptyset$ and

$$
f(x)-\mu \geq \lambda d\left(x, S_{\mu}\right) \text { for all } x \in X,
$$

where, for $\emptyset \neq Y \subseteq X$ and $x \in X$,

$$
d(x, Y):=\inf \{\rho(x, y): y \in Y\}
$$

denotes the distance from $x$ to $Y$.

This is an important notion with many applications in optimization theory, see, for instance, 43, 44, 45.

Remark 3.12. Adopting the convention $\inf \emptyset=+\infty$, the validity of (3.14) implies $S_{\mu}(f) \neq \emptyset$, i.e., $f$ attains its minimum on $X$.

The implication $1 \Rightarrow 2$ from the following theorem was proved by $\mathrm{Ng}$ [152 supposing that $X$ is a Banach space. The completeness result, i.e. the implication $3 \Rightarrow 1$, was obtained by Huang [86].

Theorem 3.13. Let $(X, \rho)$ be a metric space. The following are equivalent.

1. The metric space $X$ is complete.

2. Every bounded below lsc proper function $f: X \rightarrow \mathbb{R} \cup\{\infty\}$ with $\mu=\inf f(X)$ for which there exist a sequence $\left(\mu_{n}\right)$ in $\mathbb{R}$ and $\lambda>0$ satisfying the conditions

(i) $\quad \mu<\mu_{n+1}<\mu_{n}$ for all $n \in \mathbb{N}$;

$$
\begin{aligned}
& \text { (ii) } \lim _{n \rightarrow \infty} \mu_{n}=\mu \text {; } \\
& \text { (iii) } \lim _{n \rightarrow \infty} \lambda d\left(x, S_{\mu_{n}}\right) \leq f(x)-\mu \text { for all } x \in X \backslash S_{\mu},
\end{aligned}
$$

has global weak sharp minima with constant $\lambda>0$. 
3. Every Lipschitz function $f: X \rightarrow \mathbb{R}$ satisfying (3.15) with $\mu=0$ attains its minimum on $X$.

Proof. $1 \Rightarrow 2$. Let $\lambda>0$ and $\left(\mu_{n}\right)$ a sequence satisfying the conditions (3.15).

We show that $S_{\mu} \neq \emptyset$ and that, for every $0<\gamma<\lambda$,

$$
\gamma d\left(x, S_{\mu}\right) \leq f(x)-\mu \text { for all } x \in X,
$$

which will imply (3.14).

Let $0<\gamma<\lambda$ and $x \in X$. By EkVP (Theorem 2.2) there exists $z \in X$ such that

(i) $f(z)+\gamma \rho(x, z) \leq f(x)$;

(ii) $f(z)<f\left(x^{\prime}\right)+\gamma \rho\left(x^{\prime}, z\right)$ for all $x^{\prime} \in X \backslash\{z\}$.

The set $S_{\mu_{n}}$ is obviously nonempty and closed (because $f$ is lsc). By (3.15).(i),

(i) $S_{\mu_{n+1}} \subseteq S_{\mu_{n}}$ and

(ii) $0 \leq d\left(x^{\prime}, S_{\mu_{n}}\right) \leq d\left(x^{\prime}, S_{\mu_{n+1}}\right) \quad$ for all $x^{\prime} \in X$,

for all $n \in \mathbb{N}$.

If $f(z)=\mu$, i.e. $z \in S_{\mu}$, then, by (3.17).(i),

$$
\gamma d\left(x, S_{\mu}\right) \leq \gamma \rho(x, z) \leq f(x)-f(z)=f(x)-\mu,
$$

i.e. (3.16) holds.

If $\lim _{n \rightarrow \infty} d\left(z, S_{\mu_{n}}\right)=0$, then $d\left(z, S_{\mu_{n}}\right)=0$ for all $n$ (by (3.18).(ii)). Since $S_{\mu_{n}}$ is closed this implies $z \in S_{\mu_{n}}$, that is,

$$
\mu \leq f(z) \leq \mu_{n} \text { for all } n \in \mathbb{N},
$$

and so, by (3.15).(ii), $f(z)=\mu$ and (3.16) holds.

If $f(z)>\mu$, then

and so

$$
\lim _{n \rightarrow \infty} d\left(z, S_{\mu_{n}}\right)>0
$$

$$
d\left(z, S_{\mu_{n}}\right)>0 \Longleftrightarrow z \notin S_{\mu_{n}}
$$

for all sufficiently large $n$ (i.e., for all $n \geq n_{0}$ for some $n_{0} \in \mathbb{N}$ ).

By (3.17).(ii),

$$
\begin{aligned}
f(z) & <f(y)+\gamma \rho(z, y) \\
& \leq \mu_{n}+\gamma \rho(z, y)
\end{aligned}
$$

for all $y \in S_{\mu_{n}}$, which implies

$$
f(z)-\mu_{n} \leq \gamma d\left(z, S_{\mu_{n}}\right)
$$

for all sufficiently large $n$.

But then, by (3.15]).(iii),

$$
\begin{aligned}
f(z)-\mu & =\lim _{n \rightarrow \infty}\left(f(z)-\mu_{n}\right) \leq \gamma \lim _{n \rightarrow \infty} d\left(z, S_{\mu_{n}}\right) \\
& <\lambda \lim _{n \rightarrow \infty} d\left(z, S_{\mu_{n}}\right) \leq f(z)-\mu
\end{aligned}
$$

a contradiction.

The implication $2 \Rightarrow 3$ is obvious. 
$3 \Rightarrow 1$. Let $\left(x_{n}\right)$ be a Cauchy sequence in $X$. As in the proof of Proposition 2.5, the function $f: X \rightarrow \mathbb{R}$ given by $f(x)=\lim _{n \rightarrow \infty} \rho\left(x, x_{n}\right), x \in X$, is well-defined, 1-Lipschitz, nenegative and $\lim _{n \rightarrow \infty} f\left(x_{n}\right)=0$, so that inf $f(X)=0$.

Let us show that $f$ satisfies the conditions (3.15) with $\mu=0$. Put $\lambda_{n}=1 / n, n \in \mathbb{N}$, so that

$$
S_{1 / n}(f)=\{x \in X: f(x) \leq 1 / n\}, \quad n \in \mathbb{N} .
$$

The sets $S_{1 / n}(f)$ have the following properties:

(i) $S_{1 / n}(f) \neq \emptyset$;

$$
\text { (ii) } S_{1 /(n+1)}(f) \subseteq S_{1 / n}(f) \text {; }
$$

(iii) $\operatorname{diam}\left(S_{1 / n}(f)\right) \leq 4 / n$,

for all $n \in \mathbb{N}$.

It is obvious that $S_{1 / n}(f) \neq \emptyset$ (because $\mu=\inf f(X)=0$ ) and that the inclusion from (3.21). (ii) holds.

For $x, y \in S_{1 / n}(f)$ let $k \in \mathbb{N}$ be such that

$$
\begin{aligned}
& \rho\left(x, x_{k}\right) \leq f(x)+\frac{1}{n} \leq \frac{2}{n} \text { and } \\
& \rho\left(y, x_{k}\right) \leq f(y)+\frac{1}{n} \leq \frac{2}{n} .
\end{aligned}
$$

Then,

$$
\rho(x, y) \leq \rho\left(x, x_{k}\right)+\rho\left(x_{k}, y\right) \leq \frac{4}{n},
$$

showing that (3.21).(iii) holds too.

The condition (3.15). (iii) with $\mu=0$.

For $x \in X$ and $n \in \mathbb{N}$ let $k \in \mathbb{N}$ be such that

$$
\begin{aligned}
& \text { (a) } \rho\left(x, x_{k}\right) \leq f(x)+\frac{1}{n} \\
& \text { (b) } \rho\left(x_{k}, x_{k+i}\right) \leq \frac{1}{n} \text { for all } i \in \mathbb{N} .
\end{aligned}
$$

This is possible by the definition of the function $f$ and the fact that the sequence $\left(x_{n}\right)$ is Cauchy. Letting $i \rightarrow \infty$ in (b) one obtains $f\left(x_{k}\right) \leq 1 / n$, that is, $x_{k} \in S_{1 / n}(f)$. But then, by (a),

$$
d\left(x, S_{1 / n}(f)\right) \leq \rho\left(x, x_{k}\right) \leq f(x)+\frac{1}{n},
$$

for all $n \in \mathbb{N}$, so that

$$
\lim _{n \rightarrow \infty} d\left(x, S_{1 / n}(f)\right) \leq f(x) .
$$

By hypothesis $S_{0}(f) \neq \emptyset$. Since $S_{0}(f) \subseteq S_{1 / n}(f)$ for all $n \in \mathbb{N}$, the condition (3.21).(iii) implies $\operatorname{diam}\left(S_{0}(f)\right)=0$, and so $S_{0}(f)=\{z\}$ for some $z \in X$.

This implies

$$
0=f(z)=\lim _{n \rightarrow \infty} \rho\left(z, x_{n}\right)
$$

that is, $\left(x_{n}\right)$ converges to $z$, proving the completeness of $X$.

Remark 3.14. The proof of $1 \Rightarrow 2$ given in [152] contains a flaw. Namely, one affirms that

$$
f(z)-\mu_{n}>\gamma d\left(z, S_{\mu_{n}}\right)
$$


for sufficiently large $n$, which, by (3.20), is impossible. The proof given here follows the ideas from [152].

\section{EKVP IN QUASI-METRIC SPACES}

This section is concerned with Ekeland Variational Principle and Caristi's fixed point theorem in the context of quasi-metric spaces.

4.1. Quasi-metric spaces. We shall briefly present the fundamental properties of quasimetric spaces. Details and references can be found in the book [49].

Definition 4.1. A quasi-semimetric on an arbitrary set $X$ is a mapping $\rho: X \times X \rightarrow[0 ; \infty)$ satisfying the following conditions:

$$
\begin{array}{ll}
\text { (QM1) } & \rho(x, y) \geq 0, \quad \text { and } \quad \rho(x, x)=0 ; \\
\text { (QM2) } & \rho(x, z) \leq \rho(x, y)+\rho(y, z),
\end{array}
$$

for all $x, y, z \in X$. If, further,

$$
\text { (QM3) } \quad \rho(x, y)=\rho(y, x)=0 \Rightarrow x=y,
$$

for all $x, y \in X$, then $\rho$ is called a quasi-metric. The pair $(X, \rho)$ is called a quasi-semimetric space, respectively a quasi-metric space. The conjugate of the quasi-semimetric $\rho$ is the quasi-semimetric $\bar{\rho}(x, y)=\rho(y, x), x, y \in X$. The mapping $\rho^{s}(x, y)=\max \{\rho(x, y), \bar{\rho}(x, y)\}$, $x, y \in X$, is a semimetric on $X$ which is a metric if and only if $\rho$ is a quasi-metric.

If $(X, \rho)$ is a quasi-semimmetric space, then for $x \in X$ and $r>0$ we define the balls in $X$ by the formulae

$$
\begin{aligned}
B_{\rho}(x, r) & =\{y \in X: \rho(x, y)<r\}-\text { the open ball, and } \\
B_{\rho}[x, r] & =\{y \in X: \rho(x, y) \leq r\}-\text { the closed ball. }
\end{aligned}
$$

The topology $\tau_{\rho}$ of a quasi-semimetric space $(X, \rho)$ can be defined starting from the family $\mathcal{V}_{\rho}(x)$ of neighborhoods of an arbitrary point $x \in X$ :

$$
\begin{aligned}
V \in \mathcal{V}_{\rho}(x) & \Longleftrightarrow \exists r>0 \text { such that } B_{\rho}(x, r) \subseteq V \\
& \Longleftrightarrow \exists r^{\prime}>0 \text { such that } B_{\rho}\left[x, r^{\prime}\right] \subseteq V .
\end{aligned}
$$

The convergence of a sequence $\left(x_{n}\right)$ to $x$ with respect to $\tau_{\rho}$, called $\rho$-convergence and denoted by $x_{n} \stackrel{\rho}{\rightarrow} x$, can be characterized in the following way

$$
x_{n} \stackrel{\rho}{\rightarrow} x \Longleftrightarrow \rho\left(x, x_{n}\right) \rightarrow 0 \text {. }
$$

Also

$$
x_{n} \stackrel{\bar{\rho}}{\rightarrow} x \Longleftrightarrow \bar{\rho}\left(x, x_{n}\right) \rightarrow 0 \Longleftrightarrow \rho\left(x_{n}, x\right) \rightarrow 0 .
$$

As a space equipped with two topologies, $\tau_{\rho}$ and $\tau_{\bar{\rho}}$, a quasi-metric space can be viewed as a bitopological space in the sense of Kelly [110]. The problem of quasi-metrizability of topologies is discussed in [122].

An important example of quasi-metric space is the following.

Example 4.2. On $X=\mathbb{R}$ let $q(x, y)=(y-x)^{+}$, where $\alpha^{+}$stands for the positive part of a real number $\alpha$. Then $\bar{q}(x, y)=(x=y)^{+}$and $q^{s}(x, y)=|y-x|$. The balls are given by

$$
B_{q}(x, r)=(-\infty, x+r) \quad \text { and } \quad B_{\bar{q}}(x, r)=(x-r, \infty) .
$$


The following topological properties are true for quasi-semimetric spaces.

Proposition 4.3 (see [49]). If $(X, \rho)$ is a quasi-semimetric space, then

(1) The ball $B_{\rho}(x, r)$ is $\tau_{\rho}$-open and the ball $B_{\rho}[x, r]$ is $\tau_{\bar{\rho}}$-closed. The ball $B_{\rho}[x, r]$ need not be $\tau_{\rho}$-closed.

(2) If $\rho$ is a quasi-metric, then the topology $\tau_{\rho}$ is $T_{0}$, but not necessarily $T_{1}$ (and so nor $T_{2}$ as in the case of metric spaces).

The topology $\tau_{\rho}$ is $T_{1}$ if and only if $\rho(x, y)>0$ whenever $x \neq y$.

(3) For every fixed $x \in X$, the mapping $\rho(x, \cdot): X \rightarrow(\mathbb{R},|\cdot|)$ is $\tau_{\rho}$-usc and $\tau_{\bar{\rho}}$-lsc. For every fixed $y \in X$, the mapping $\rho(\cdot, y): X \rightarrow(\mathbb{R},|\cdot|)$ is $\tau_{\rho}$-lsc and $\tau_{\bar{\rho}}$-usc.

(4) If the mapping $\rho(x, \cdot): X \rightarrow(\mathbb{R},|\cdot|)$ is $\tau_{\rho}$-continuous for every $x \in X$, then the topology $\tau_{\rho}$ is regular.

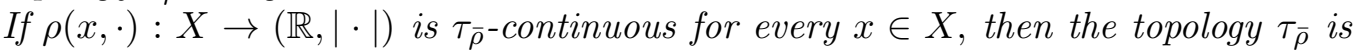
semi-metrizable.

4.2. Completeness in quasi-metric spaces. The lack of symmetry in the definition of quasi-metric and quasi-uniform spaces causes a lot of troubles, mainly concerning completeness, compactness and total boundedness in such spaces. There are a lot of completeness notions in quasi-metric and in quasi-uniform spaces, all agreeing with the usual notion of completeness in the case of metric or uniform spaces, each of them having its advantages and weaknesses.

As in what follows we shall work only with one of these notions, we shall present only it, referring to [49]) for other notions of Cauchy sequence and for their properties.

A sequence $\left(x_{n}\right)$ in $(X, \rho)$ is called

(a) left $\rho$-K-Cauchy if for every $\varepsilon>0$ there exists $n_{\varepsilon} \in \mathbb{N}$ such that

$$
\begin{aligned}
& \forall n, m, \quad \text { with } n_{\varepsilon} \leq n<m, \quad \rho\left(x_{n}, x_{m}\right)<\varepsilon \\
\Longleftrightarrow & \forall n \geq n_{\varepsilon}, \forall k \in \mathbb{N}, \quad \rho\left(x_{n}, x_{n+k}\right)<\varepsilon .
\end{aligned}
$$

Similarly, a sequence $\left(x_{n}\right)$ in $(X, \rho)$ is called

$\left(\mathrm{a}^{\prime}\right)$ right $\rho$-K-Cauchy if for every $\varepsilon>0$ there exists $n_{\varepsilon} \in \mathbb{N}$ such that

$$
\begin{aligned}
& \forall n, m, \quad \text { with } n_{\varepsilon} \leq n<m, \quad \rho\left(x_{m}, x_{n}\right)<\varepsilon \\
\Longleftrightarrow & \forall n \geq n_{\varepsilon}, \forall k \in \mathbb{N}, \quad \rho\left(x_{n+k}, x_{n}\right)<\varepsilon .
\end{aligned}
$$

Remarks 4.4. Let $(X, \rho)$ be a quasi-semimetric space.

1. Obviously, a sequence is left $\rho$ - $K$-Cauchy if and only if it is right $\bar{\rho}$ - $K$-Cauchy.

2 . Let $\left(x_{n}\right)$ be a left $\rho$-K-Cauchy sequence. If $\left(x_{n}\right)$ contains a subsequence which is $\tau(\rho)(\tau(\bar{\rho}))$-convergent to some $x \in X$, then the sequence $\left(x_{n}\right)$ is $\tau(\rho)$ (resp. $\tau(\bar{\rho}))$-convergent to $x([49$, P. 1.2.4]).

3. If a sequence $\left(x_{n}\right)$ in $X$ satisfies $\sum_{n=1}^{\infty} \rho\left(x_{n}, x_{n+1}\right)<\infty\left(\sum_{n=1}^{\infty} \rho\left(x_{n+1}, x_{n}\right)<\infty\right)$, then it is left (right)- $\rho$ - $K$-Cauchy.

4. There are examples showing that a $\rho$-convergent sequence need not be left $\rho$ - $K$ Cauchy, showing that in the asymmetric case the situation is far more complicated than in the symmetric one (see [49, Section 1.2]).

5. If each convergent sequence in a regular quasi-metric space $(X, \rho)$ admits a left $K$-Cauchy subsequence, then $X$ is metrizable ([49, P. 1.2.1]).

A quasi-metric space $(X, \rho)$ is called left $\rho$ - $K$-complete if every left $\rho$ - $K$-Cauchy sequence is $\rho$-convergent, with the corresponding definition of the right $\rho$-K-completeness. The 
quasi-metric space $(X, \rho)$ is called left (right) Smyth complete if every left (right) $\rho$ - $K$ Cauchy sequence is $\rho^{s}$-convergent and bicomplete if the associated metric space $\left(X, \rho^{s}\right)$ is complete.

Remark 4.5. In spite of the obvious fact that left $\rho$ - $K$-Cauchy is equivalent to right $\bar{\rho}$ - $K$-Cauchy, left $\rho$ - $K$ - and right $\bar{\rho}-K$-completeness do not agree, due to the fact that right $\bar{\rho}$-completeness means that every left $\rho$-Cauchy sequence converges in $(X, \bar{\rho})$, while left $\rho$-completeness means the convergence of such sequences in the space $(X, \rho)$.

Also, it is easy to check that Smyth completeness (left or right) of a quasi-metric space $(X, \rho)$ implies the completeness of the associated metric space $\left(X, \rho^{s}\right)$ (i.e. the bicompleteness of the quasi-metric space $(X, \rho))$.

Example 4.6. The spaces $(\mathbb{R}, q)$ and $(\mathbb{R}, \bar{q})$ from Example 4.2 are not right $K$-complete. The sequence $x_{n}=n, n \in \mathbb{N}$, is right $q$ - $K$-Cauchy and not convergent in $(\mathbb{R}, q)$ and the sequence $y_{n}=-n, n \in \mathbb{N}$, is right $\bar{q}$ - $K$-Cauchy and not convergent in $(\mathbb{R}, \bar{q})$.

Indeed, $q\left(x_{n+k}, x_{n}\right)=(n-n-k)^{+}=0$ for all $n, k \in \mathbb{N}$. For $x \in \mathbb{R}$ let $n_{x} \in \mathbb{N}$ be such that $n_{x}>x$. Then $q\left(x, x_{n}\right)=n-x \geq n_{x}-x>0$ for all $n \geq n_{x}$. The case of the space $(\mathbb{R}, \bar{q})$ and of the sequence $y_{n}=-n, n \in \mathbb{N}$, can be treated similarly.

4.3. Variational principles, fixed points and completeness. The following version of EkVP in quasi-metric spaces was proved in [47].

Theorem 4.7 (Ekeland Variational Principle). Suppose that $(X, \rho)$ is a $T_{1}$ quasi-metric space and $f: X \rightarrow \mathbb{R} \cup\{\infty\}$ is a bounded below proper function. For given $\varepsilon>0$ let $x_{\varepsilon} \in X$ be such that

$$
f\left(x_{\varepsilon}\right) \leq \inf f(X)+\varepsilon .
$$

(1) If $(X, \rho)$ is right $\rho$-K-complete and $f$ is $\rho$-lsc, then for every $\lambda>0$ there exists $z=z_{\varepsilon, \lambda} \in X$ such that

(a) $f(z)+\frac{\varepsilon}{\lambda} \rho\left(z, x_{\varepsilon}\right) \leq f\left(x_{\varepsilon}\right)$;

(b) $\rho\left(z, x_{\varepsilon}\right) \leq \lambda$;

(c) $\forall x \in X \backslash\{z\}, \quad f(z)<f(x)+\frac{\varepsilon}{\lambda} \rho(x, z)$.

(2) If $(X, \rho)$ is right $\bar{\rho}$-K-complete and $f$ is $\bar{\rho}$-lsc, then for every $\lambda>0$ there exists $z=z_{\varepsilon, \lambda} \in X$ such that

$\left(\mathrm{a}^{\prime}\right) \quad f(z)+\frac{\varepsilon}{\lambda} \rho\left(x_{\varepsilon}, z\right) \leq f\left(x_{\varepsilon}\right)$;

$\left(\mathrm{b}^{\prime}\right) \quad \rho\left(x_{\varepsilon}, z\right) \leq \lambda$;

(c') $\forall x \in X \backslash\{z\}, \quad f(z)<f(x)+\frac{\varepsilon}{\lambda} \rho(z, x)$.

Again, taking $\lambda=1$ in Theorem 4.7, one obtains the weak form of EkVP in quasi-metric spaces.

Corollary 4.8 (Ekeland's Variational Principle - weak form). Suppose that $(X, \rho)$ is a $T_{1}$ quasi-metric space and $f: X \rightarrow \mathbb{R} \cup\{\infty\}$ is a bounded below proper function.

(1) If $X$ is right $\rho$-K-complete and $f$ is $\rho$-lsc, then for every $\varepsilon>0$ there exists an element $y_{\varepsilon} \in X$ such that

(i) $f\left(y_{\varepsilon}\right) \leq \inf f(X)+\varepsilon$,

(ii) $\forall x \in X \backslash\left\{y_{\varepsilon}\right\}, \quad f\left(y_{\varepsilon}\right)<f(x)+\varepsilon \rho\left(x, y_{\varepsilon}\right)$. 
(2) If $X$ is right $\bar{\rho}$-K-complete and $f$ is $\bar{\rho}$-lsc, then for every $\varepsilon>0$ there exists an element $y_{\varepsilon} \in X$ such that

(i) $f\left(y_{\varepsilon}\right) \leq \inf f(X)+\varepsilon$,

(ii) $\forall x \in X \backslash\left\{y_{\varepsilon}\right\}, \quad f\left(y_{\varepsilon}\right)<f(x)+\varepsilon \rho\left(y_{\varepsilon}, x\right)$.

Caristi's fixed point theorem version in quasi-metric spaces is the following.

Theorem 4.9 (Caristi-Kirk Fixed Point Theorem, 47]). Let $(X, \rho)$ be a $T_{1}$ quasi-metric space, $f: X \rightarrow X$ and $\varphi: X \rightarrow \mathbb{R}$.

(1) If $X$ is right $\rho$-K-complete, $\varphi$ is bounded below and $\rho$-lsc and the mapping $f$ satisfies the condition

$$
\rho(f(x), x) \leq \varphi(x)-\varphi(f(x)), x \in X,
$$

then $f$ has a fixed point in $X$.

(2) If $X$ is right $\bar{\rho}$-K-complete, $\varphi$ is bounded below and $\bar{\rho}$-lsc and the mapping $f$ satisfies the condition

$$
\rho(x, f(x)) \leq \varphi(x)-\varphi(f(x)), x \in X,
$$

then $f$ has a fixed point in $X$.

In this case we have also a set-valued version.

Theorem 4.10 (Caristi-Kirk Fixed Point Theorem - set-valued version, [47]). Let $(X, \rho)$ be a $T_{1}$ quasi-metric space, $F: X \rightrightarrows X$ a set-valued mapping such that $F(x) \neq \emptyset$ for every $x \in X$, and $\varphi: X \rightarrow \mathbb{R}$.

(1) If $X$ is right $\rho$-K-complete, $\varphi$ is bounded below and $\rho$-lsc and the mapping $F$ satisfies the condition

$$
\forall x \in X, \forall y \in F(x), \quad \rho(y, x) \leq \varphi(x)-\varphi(y),
$$

then $F$ has a fixed point in $X$.

(2) If $X$ is right $\bar{\rho}$-K-complete, $\varphi$ is bounded below and $\bar{\rho}$-lsc and the mapping $F$ satisfies the condition

$$
\forall x \in X, \forall y \in F(x), \quad \rho(x, y) \leq \varphi(x)-\varphi(y),
$$

then $F$ has a fixed point in $X$.

As in the symmetric case, the weak form of Ekeland Variational Principle is equivalent to Caristi's fixed point theorem, [47].

Proposition 4.11. Let $(X, \rho)$ be a $T_{1}$ quasi-metric space. Consider the following assertions.

(wEk) For any $\rho$-closed subset $Y$ of $X$, for every bounded below $\rho$-lsc proper function $f: Y \rightarrow \mathbb{R} \cup\{\infty\}$ and for every $\varepsilon>0$ there exists $x_{\varepsilon} \in Y$ such that

$$
\forall y \in Y \backslash\left\{x_{\varepsilon}\right\}, \quad f\left(x_{\varepsilon}\right)<f(y)+\varepsilon \rho\left(y, x_{\varepsilon}\right) .
$$

(C) For every $\rho$-closed subset $Y$ of $X$ and for any $\rho$-lsc function $\varphi: Y \rightarrow \mathbb{R}$, any function $g: Y \rightarrow Y$ satisfying (4.9) on $Y$ has a fixed point.

Then $(\mathrm{wEk}) \Longleftrightarrow(\mathrm{C})$.

As we have seen, in the case of a metric space $X$, the validity of the weak form of Ekeland Variational Principle implies the completeness of $X$ (Proposition 2.5). The following proposition contains some partial converse results in the quasi-metric case. 
Proposition 4.12 (47]). Let $(X, \rho)$ be a $T_{1}$ quasi-metric space.

(1) If for every $\rho$-lsc function $f: X \rightarrow \mathbb{R}$ and for every $\varepsilon>0$ there exists $y_{\varepsilon} \in X$ such that

$$
\forall x \in X, \quad f\left(y_{\varepsilon}\right) \leq f(x)+\varepsilon \rho\left(y_{\varepsilon}, x\right)
$$

then the quasi-metric space $X$ is left $\rho$-K-complete.

(2) If for every $\bar{\rho}$-lsc function $f: X \rightarrow \mathbb{R}$ and for every $\varepsilon>0$ there exists $y_{\varepsilon} \in X$ such that

$$
\forall x \in X, \quad f\left(y_{\varepsilon}\right) \leq f(x)+\varepsilon \rho\left(x, y_{\varepsilon}\right),
$$

then the quasi-metric space $X$ is left $\bar{\rho}$-K-complete.

Proof. The proof is similar to that of Proposition 2.5. taking care of the fact that a quasimetric has weaker continuity properties than a metric (see Proposition 4.3).

To prove (1), suppose that $\left(x_{n}\right)$ is a left $\rho$ - $K$-Cauchy sequence in $X$. We show first that, for every $n \in \mathbb{N}$, the sequence $\left(\rho\left(x, x_{n}\right)\right)$ is bounded. Indeed, if $n_{1} \in \mathbb{N}$ is such that $\rho\left(x_{n_{1}}, x_{n_{1}+k}\right) \leq 1$ for all $k \in \mathbb{N}$, then

$$
\rho\left(x, x_{n_{1}+k}\right) \leq \rho\left(x, x_{n_{1}}\right)+\rho\left(x_{n_{1}}, x_{n_{1}+k}\right) \leq \rho\left(x, x_{n_{1}}+1,\right.
$$

for all $k \in \mathbb{N}$, proving the boundedness of the sequence $\left(\rho\left(x, x_{n}\right)\right)$.

Consequently, the function $f: X \rightarrow[0, \infty)$ given by

$$
f(x)=\limsup _{n \rightarrow \infty} \rho\left(x, x_{n}\right), \quad x \in X,
$$

is well defined.

For $x, x^{\prime} \in X$,

$$
\rho\left(x, x_{n}\right) \leq \rho\left(x, x^{\prime}\right)+\rho\left(x^{\prime}, x_{n}\right),
$$

for all $n \in \mathbb{N}$. Passing to limsup in both sides of this inequality one obtains

$$
f\left(x^{\prime}\right) \geq f(x)-\rho\left(x, x^{\prime}\right) .
$$

Then for every $\varepsilon>0, \rho\left(x, x^{\prime}\right)<\varepsilon$ implies $f\left(x^{\prime}\right)>f(x)-\varepsilon$, proving that $f$ is $\rho$-lsc at every $x \in X$.

Similarly,

$$
\rho\left(x^{\prime}, x_{n}\right) \leq \rho\left(x^{\prime}, x\right)+\rho\left(x, x_{n}\right), \quad n \in \mathbb{N},
$$

implies

$$
f\left(x^{\prime}\right) \leq f(x)+\rho\left(x^{\prime}, x\right),
$$

from which follows that the function $f$ is $\bar{\rho}$-usc at every $x$.

We show now that

$$
\lim _{n \rightarrow \infty} f\left(x_{n}\right)=0 .
$$

Indeed, for every $\varepsilon>0$ there exists $n_{\varepsilon} \in \mathbb{N}$ such that

$$
\forall n \geq n_{\varepsilon}, \forall k \in \mathbb{N}, \quad \rho\left(x_{n}, x_{n+k}\right)<\varepsilon,
$$

implying

$$
\forall n \geq n_{\varepsilon}, \quad 0 \leq f\left(x_{n}\right)=\underset{k}{\limsup } \rho\left(x_{n}, x_{n+k}\right) \leq \varepsilon,
$$

that is $\lim _{n} f\left(x_{n}\right)=0$. 
Let now $y \in X$ satisfying (4.14) for $\varepsilon=1 / 2$. Taking $x=x_{n}$ it follows

$$
\forall n \in \mathbb{N}, \quad f(y) \leq f\left(x_{n}\right)+\frac{1}{2} \rho\left(y, x_{n}\right) .
$$

Passing to limsup and taking into account (4.16) one obtains

$$
f(y)=\frac{1}{2} f(y)
$$

that implies $f(y)=0$. Since

$$
f(y)=0 \Longleftrightarrow \limsup _{n} \rho\left(y, x_{n}\right)=0 \Longleftrightarrow \lim _{n} \rho\left(y, x_{n}\right)=0,
$$

it follows that the sequence $\left(x_{n}\right)$ is $\rho$-convergent to $y$, proving the left $\rho$ - $K$-completeness of the quasi-metric space $X$.

The proof of (2) is similar, working with the function $g: X \rightarrow[0, \infty)$ given by

$$
g(x)=\limsup _{n} \rho\left(x_{n}, x\right), \quad x \in X,
$$

which is $\bar{\rho}$-lsc and $\rho$-usc.

Remark 4.13. Note that Proposition 4.12 does not contain a proper converse (in the sense of completeness) of the weak Ekeland Principle. We have in fact a kind of "cross" converse, as can be seen from the following explanations.

From Corollary 4.8,2 it follows that if the quasi-metric space $(X, \rho)$ is right $\bar{\rho}-K$ complete, then for every $\bar{\rho}$-lsc function $f: X \rightarrow \mathbb{R}$ and every $\varepsilon>0$, there exists a point $y_{\varepsilon} \in X$ satisfying (4.14).

On the other side, the fulfillment of (4.14) for any $\rho$-lsc function implies the left $\rho-K$ completeness of the quasi-metric space $(X, \rho)$.

Of course that, in the metric case, both of these conditions reduce to the completeness of $X$.

Taking into account the fact that a sequence $\left(x_{n}\right)$ in $X$ is right $\bar{\rho}$ - $K$-Cauchy if and only if it is left $\rho$ - $K$-Cauchy one obtains the following completeness results:

$(X, \rho)$ is right $\bar{\rho}$ - $K$-complete $\Longleftrightarrow$

$\forall\left(x_{n}\right)$ a left $\rho$-K-Cauchy sequence in $X, \exists x \in X$ such that $x_{n} \stackrel{\bar{\rho}}{\rightarrow} x$,

while

$$
\begin{aligned}
& (X, \rho) \text { is left } \rho \text { - } K \text {-complete } \Longleftrightarrow \\
& \forall\left(x_{n}\right) \text { a left } \rho \text {-K-Cauchy sequence in } X, \exists x \in X \text { such that } x_{n} \stackrel{\rho}{\rightarrow} x .
\end{aligned}
$$

The right converse was given by Karapinar and Romaguera [104. To do this they need to slightly modify the notion of lsc function.

Let $(X, \rho)$ be a quasi-metric space. A proper function $f: X \rightarrow \mathbb{R} \cup\{\infty\}$, is called nearly $\rho$-lsc at $x \in X$ if $f(x) \leq \liminf _{n} f\left(x_{n}\right)$ for every sequence $\left(x_{n}\right)$ of distinct points in $X$ which is $\rho$-convergent to $x$.

It is clear that a $\rho$-lsc function is nearly $\rho$-lsc and if the topology $\tau_{\rho}$ is $T_{1}$ (equivalent to $\rho(x, y)>0$ for all distinct points $x, y \in X)$, then the converse is also true. The following simple example shows that these notions are different in $T_{0}$ quasi-metric spaces.

Example 4.14. Let $X=\{0,1\}, \rho(0,0)=\rho(0,1)=\rho(1,1)=0$ and $\rho(1,0)=1$. Then every function $f: X \rightarrow \mathbb{R} \cup\{\infty\}$ is nearly $\rho$-lsc (there are no sequences formed of distinct points), but the function $f(0)=1, f(1)=0$ is not $\rho$-lsc at $x=0$. 
Indeed, $x_{n}=1$ satisfies $\rho\left(0, x_{n}\right)=0 \rightarrow 0, f\left(x_{n}\right)=0$ and $f(0)=1>0=\liminf _{n} f\left(x_{n}\right)$.

Theorem 4.15. For a quasi-semimetric space $(X, \rho)$ the following conditions are equivalent.

(1) $(X, \rho)$ is right $K$-sequentially complete.

(2) For every self mapping $T$ of $X$ and every bounded below and nearly $\rho$-lsc proper function $\varphi: X \rightarrow \mathbb{R} \cup\{\infty\}$ satisfying the inequality

$$
\rho(T(x), x)+\varphi(T(x)) \leq \varphi(x),
$$

for all $x \in X$, there exists $z=z_{T, \varphi} \in X$ such that $\varphi(z)=\varphi(T(z))$.

(3) For every bounded below and nearly $\rho$-lsc proper function $f: X \rightarrow \mathbb{R} \cup\{\infty\}$ and for every $\varepsilon>0$ there exists $y_{\varepsilon} \in X$ such that

$$
\begin{aligned}
& \text { (i) } f\left(y_{\varepsilon}\right) \leq \inf f(X)+\varepsilon ; \\
& \text { (ii) } f\left(y_{\varepsilon}\right)<f(x)+\varepsilon \rho\left(x, y_{\varepsilon}\right) \text { for all } x \in X \backslash \overline{\left\{y_{\varepsilon}\right\}} \text { and } \\
& \text { (iii) } f\left(y_{\varepsilon}\right) \leq f(x) \text { for all } x \in \overline{\left\{y_{\varepsilon}\right\}} \text {. }
\end{aligned}
$$

Proof. We shall present only the proof of the implication $(3) \Rightarrow(1)$.

We proceed by contradiction. Suppose that the space $(X, \rho)$ is not right $K$-complete. Then there exists a right $K$-Cauchy sequence $\left(x_{n}\right)$ in $X$ which has no limit. This implies that $\left(x_{n}\right)$ has no convergent subsequences, see Remarks 4.4 .

We shall distinct two situations.

Suppose that

$$
\exists m, \forall k \geq m, \exists n_{k}>k, \rho\left(x_{n_{k}}, x_{k}\right)>0 .
$$

Then, for $n_{1}=m$ there exists $n_{2}>n_{1}$ such that $\rho\left(x_{n_{2}}, x_{n_{1}}\right)>0$. Taking $k=n_{2}$ it follows the existence of $n_{3}>n_{2}$ such that $\rho\left(x_{n_{3}}, x_{n_{2}}\right)>0$. Continuing in this manner we obtain a sequence $n_{1}<n_{2}<\ldots$ such that $\rho\left(x_{n_{k+1}}, x_{n_{k}}\right)>0$ for all $k \in \mathbb{N}$.

Passing to a further subsequence, if necessary, and relabeling, we can suppose that

$$
0<\rho\left(x_{n+1}, x_{n}\right)<\frac{1}{2^{n+1}},
$$

for all $n \in \mathbb{N}$.

If (4.18) does not hold, then

$$
\forall m, \exists k \geq m, \text { such that } \forall n>k, \rho\left(x_{n}, x_{k}\right)=0 .
$$

For $m=1$ let $k=n_{1} \geq 1$ be such that $\rho\left(x_{n}, x_{n_{1}}\right)=0$ for all $n>n_{1}$. Now, for $m=1+n_{1}$ let $n_{2}>n_{1}$ be such that $\rho\left(x_{n}, x_{n_{2}}\right)=0$ for all $n>n_{2}$. It follows $\rho\left(x_{n_{2}}, x_{n_{1}}\right)=0$.

Continuing in this manner we obtain a sequence $n_{1}<n_{2}<\ldots$ such that $\rho\left(x_{n_{k+1}}, x_{n_{k}}\right)=$ 0 for all $k \in \mathbb{N}$.

Relabeling, if necessary, we can suppose that the sequence $\left(x_{n}\right)$ satisfies

$$
\rho\left(x_{n+1}, x_{n}\right)=0
$$

for all $n \in \mathbb{N}$.

Put

$$
B:=\left\{x_{n}: n \in \mathbb{N}\right\},
$$


and define $f: X \rightarrow \mathbb{R}$ by

$$
f(x)= \begin{cases}\frac{1}{2^{n-1}} & \text { if } x=x_{n} \text { for some } n \in \mathbb{N}, \\ 2 & \text { for } x \in X \backslash B .\end{cases}
$$

The function $f$ is nearly $\rho$-lsc. Indeed, let $x \in X$ and $\left(y_{n}\right)$ a sequence of distinct points in $X$ converging to $x$. If the set $\left\{n \in \mathbb{N}: y_{n} \in B\right\}$ would be infinite, then there would exist the natural numbers $m_{1}<m_{2}<\ldots$ and $n_{1}<n_{2}<\ldots$ such that $y_{m_{k}}=x_{n_{k}}, k \in \mathbb{N}$. But, this would imply that $\left(x_{n}\right)$ has a subsequence $\left(x_{n_{k}}\right)$ convergent to $x$, in contradiction to the hypothesis. Consequently, $\left(y_{n}\right)$ must be eventually in $X \backslash B$, and so $f(x) \leq 2=\lim _{n} f\left(y_{n}\right)$.

For $\varepsilon=1$ let $y \in X$ satisfying the conditions (i)-(iii). Since

$$
\{x \in X: f(x) \leq \inf f(X)+1\}=\{x \in X: f(x) \leq 1\}=B,
$$

it follows $y=x_{m} \in B$ for some $m \in \mathbb{N}$.

If (4.19) holds, then

$$
f\left(x_{m+1}\right)+\rho\left(x_{m+1}, x_{m}\right)<\frac{1}{2^{m}}+\frac{1}{2^{m+1}}=\frac{3}{2^{m+1}}<\frac{1}{2^{m-1}}=f\left(x_{m}\right),
$$

showing that condition (ii) from (3) is not satisfied, that is (3) does not hold.

If (4.21) holds, then, by the triangle inequality,

$$
\rho\left(x_{m+k}, x_{m}\right) \leq \sum_{i=1}^{k} \rho\left(x_{m+i}, x_{m+i-1}\right)=0,
$$

i.e. $x_{n} \in \overline{\left\{x_{m}\right\}}$ for all $n \geq m$. By (iii),

$$
0<f\left(x_{m}\right) \leq f\left(x_{n}\right)=\frac{1}{2^{n-1}},
$$

for all $n \geq m$. Since $2^{n-1}<f\left(x_{m}\right)$ for sufficiently large $n$, this leads to a contradiction.

Remark 4.16. In the proof of Theorem 2 in [104] the possibility that $\rho\left(x_{n+1}, x_{n}\right)=0$ for all $n \in \mathbb{N}$ (when one can not use $x_{m+1}$ to obtain the contradiction from (4.23)) is not discussed. So the proof given above fills in this gap.

4.4. Smyth completeness. We present now some results on Caristi FPT and Smyth completeness in quasi-metric spaces obtained by Romaguera and Tirado [171.

Let $(X, \rho)$ be a quasi-metric space, $\varphi: X \rightarrow[0, \infty)$ and $T: X \rightarrow X$ such that

$$
\rho(x, T x) \leq \varphi(x)-\varphi(T x),
$$

for all $x \in X$.

The mapping $T$ is called $\bar{\rho}$-Caristi if $\varphi$ is $\bar{\rho}$-lsc and $\rho^{s}$-Caristi if $\varphi$ is $\rho^{s}$-lsc.

Theorem 4.17 (Romaguera and Tirado [171]). Let $(X, \rho)$ be a quasi-metric space.

(1) If $(X, \rho)$ is right $\bar{\rho}-K$-complete, then every $\bar{\rho}$-Caristi map on $X$ has a fixed point.

(2) If $(X, \rho)$ is right $\rho$-K-complete, then every $\rho$-Caristi map on $X$ has a fixed point.

(3) The quasi-metric space $(X, \rho)$ is right $\bar{\rho}$-Smyth complete if and only if every $\rho^{s}$ Caristi map has a fixed point.

Remark 4.18. Some versions of Ekeland Variational Principle in asymmetric locally convex spaces were proved in [48. The equivalence of completeness of a quasi-semimetric space with Takahashi, Ekeland and Caristi principles is discussed in [50]. Other characterizations of completeness of quasi-metric spaces are given by Romaguera and Valero 172. 
4.5. Some results of Bao, Cobzaş, Mordukhovich and Soubeyran. Inspired by the results of Dancs, Hegedüs and Medvegyev [53] (see Theorem 3.3), Bao, Cobzaş and Soubeyran [30, Bao and Soubeyran [33], and Bao and Théra [34] proved versions of Ekeland principle in quasi-semimetric spaces and obtained characterizations of completeness.

They consider a set-valued mapping attached to a function $\varphi$ and to a number $\lambda>0$, as in the following proposition.

Proposition 4.19. Let $(X, \rho)$ be a quasi-semimetric space, $\varphi: X \rightarrow \mathbb{R} \cup\{+\infty\}$ a proper function and $S_{\lambda}: X \rightrightarrows X$ the set-valued mapping defined by

$$
S_{\lambda}(x)=\{y \in X: \lambda \rho(x, y) \leq \varphi(x)-\varphi(y)\} .
$$

Then $S_{\lambda}$ enjoys the following properties:

(i) (nonemptiness) $\quad x \in S_{\lambda}(x)$ for all $x \in \operatorname{dom}(\varphi)$;

(ii) (monotonicity) $y \in S_{\lambda}(x) \Longrightarrow \varphi(y) \leq \varphi(x)$ and $S_{\lambda}(y) \subseteq S_{\lambda}(x)$.

Recall that a generalized Picard sequence corresponding for a set-valued mapping $F$ : $X \rightrightarrows X$ is a sequence $\left(x_{n}\right)$ in $X$ such that $x_{n+1} \in F\left(x_{n}\right)$ for all $n$.

Theorem 4.20. Let $(X, \rho)$ be a quasi-semimetric space, and let $\varphi: X \rightarrow \mathbb{R} \cup\{+\infty\}$ be proper. Given $x_{0} \in \operatorname{dom}(f)$ and $\lambda>0$, consider the set-valued mapping $S_{\lambda}: X \rightrightarrows X$ defined by (4.25). Assume that

(C1) (boundedness from below) $\varphi$ is bounded from below on $S_{\lambda}\left(x_{0}\right)$;

(C2) (nonempty intersection) for every generalized Picard sequence $\left(x_{n}\right)_{n \in \mathbb{N}_{0}}$ of $S_{\lambda}$ (starting with $\left.x_{0}\right)$, such that $\varphi\left(x_{n}\right)>\varphi\left(x_{n+1}\right), \forall n \in \mathbb{N}_{0}$, and $\sum_{n=0}^{\infty} \rho\left(x_{n}, x_{n+1}\right)<$ $\infty$, there exists $y \in X$ such that $S_{\lambda}(y) \subseteq S_{\lambda}\left(x_{n}\right)$ for all $n \in \mathbb{N}_{0}$, where $\mathbb{N}_{0}=\mathbb{N} \cup\{0\}$.

Then, there is a generalized Picard sequence $\left(x_{n}\right)_{n \in \mathbb{N}_{0}}$ (i.e. $x_{n+1} \in S_{\lambda}\left(x_{n}\right), \forall n \in \mathbb{N}_{0}$ ) satisfying $\sum_{n=0}^{\infty} \rho\left(x_{n}, x_{n+1}\right)<\infty, \bar{\rho}$-convergent to some $\bar{x} \in X$ such that for every $\bar{y} \in$ $S_{\lambda}(\bar{x})$ the following conditions hold:

$$
\begin{array}{ll}
\text { (i) } & \lambda \rho\left(x_{0}, \bar{y}\right) \leq \varphi\left(x_{0}\right)-\varphi(\bar{y}) ; \\
\text { (ii) } & \varphi(\bar{y})<\varphi(x)+\lambda \rho(\bar{y}, x) \quad \text { for every } x \in X \backslash S_{\lambda}(\bar{y}) \text {; } \\
\text { (iii) } & \rho(\bar{x}, \bar{y})=0, \varphi(\bar{y})=\varphi(\bar{x}) \quad \text { and } S_{\lambda}(\bar{y}) \subseteq \overline{\{\bar{y}\}^{\rho}} .
\end{array}
$$

Proof. We give a proof following the ideas from [30]. Replacing, if necessary, $\rho$ by $\lambda \rho$, we can suppose $\lambda=1$. Put also $S(x)=S_{1}(x), x \in X$.

We shall define inductively a left $\rho$ - $K$-Cauchy generalized Picard sequence which will satisfy all the requirements of the theorem.

Case I. Start with $x_{0}$ and suppose that $\alpha_{0}:=\inf \varphi\left(S\left(x_{0}\right)\right)<\varphi\left(x_{0}\right)$. Choose $x_{1} \in S\left(x_{0}\right)$ such that

$$
\alpha_{0} \leq \varphi\left(x_{1}\right)<\alpha_{0}+\frac{1}{2}\left(\varphi\left(x_{0}\right)-\alpha_{0}\right)=\frac{1}{2}\left(\alpha_{0}+\varphi\left(x_{0}\right)\right)<\varphi\left(x_{0}\right) .
$$

Suppose that we have found $x_{0}, x_{1}, \ldots, x_{n}$ satisfying $x_{k+1} \in S\left(x_{k}\right), k=0,1, \ldots, n-$ $1, \varphi\left(x_{k}\right)>\alpha_{k}:=\inf \varphi\left(S\left(x_{k}\right)\right), k=0,1, \ldots, n$, and

$$
\alpha_{k} \leq \varphi\left(x_{k+1}\right)<\alpha_{k}+\frac{1}{2}\left(\varphi\left(x_{k}\right)-\alpha_{k}\right)=\frac{1}{2}\left(\alpha_{k}+\varphi\left(x_{k}\right)\right)<\varphi\left(x_{k}\right),
$$

for $k=0,1, \ldots, n-1$.

Pick then $x_{n+1} \in S\left(x_{n}\right)$ such that

$$
\alpha_{n} \leq \varphi\left(x_{n+1}\right)<\alpha_{n}+\frac{1}{2}\left(\varphi\left(x_{n}\right)-\alpha_{n}\right)=\frac{1}{2}\left(\alpha_{n}+\varphi\left(x_{n}\right)\right)<\varphi\left(x_{n}\right) .
$$


Supposing that we can do indefinitely this procedure we find a generalized Picard sequence $x_{n+1} \in S\left(x_{n}\right), n \in \mathbb{N}_{0}$. Let us show that the conditions from (4.26) are satisfied by this sequence.

Since the sequence $\left(\varphi\left(x_{n}\right)\right)$ is strictly decreasing and bounded from below (by condition (C1)), there exists $\bar{\alpha}:=\lim _{n} \varphi\left(x_{n}\right)=\inf _{n} \varphi\left(x_{n}\right)$.

By Proposition 4.19, $x_{n+1} \in S\left(x_{n}\right)$ implies $S\left(x_{n+1}\right) \subseteq S\left(x_{n}\right) \subseteq S\left(x_{0}\right)$, so that $\alpha_{n+1} \geq$ $\alpha_{n}$, implying the existence of $\beta=\lim _{n} \alpha_{n}$. The inequalities

$$
\varphi\left(x_{n+1}\right)<\frac{1}{2}\left(\alpha_{n}+\varphi\left(x_{n}\right)\right)<\varphi\left(x_{n}\right)
$$

yield for $n \rightarrow \infty, \bar{\alpha} \leq \frac{1}{2}(\beta+\bar{\alpha}) \leq \bar{\alpha}$, implying $\beta=\bar{\alpha}$. Consequently

$$
\lim _{n} \alpha_{n}=\lim _{n} \varphi\left(x_{n}\right)=\bar{\alpha} .
$$

The inequalities

$$
q\left(x_{k}, x_{k+1}\right) \leq \varphi\left(x_{k}\right)-\varphi\left(x_{k+1}\right) \quad\left(\Longleftrightarrow x_{k+1} \in S\left(x_{k}\right)\right)
$$

yield by summation and taking into account condition $(\mathrm{C} 1)$,

$$
\begin{aligned}
& \sum_{k=0}^{n} \rho\left(x_{k}, x_{k+1}\right) \leq \varphi\left(x_{0}\right)-\varphi\left(x_{n+1}\right) \leq \\
& \leq \varphi\left(x_{0}\right)-\inf \varphi\left(S\left(x_{n+1}\right)\right) \leq \varphi\left(x_{0}\right)-\inf \varphi\left(S\left(x_{0}\right)\right),
\end{aligned}
$$

showing that $\sum_{k=0}^{\infty} \rho\left(x_{n}, x_{n+1}\right)<\infty$

Condition (C2) implies the existence of $\bar{x} \in X$ such that

$$
S(\bar{x}) \subseteq \bigcap_{n=0}^{\infty} S\left(x_{n}\right) .
$$

Since $\bar{x} \in S(\bar{x}) \subseteq S\left(x_{n}\right)$, it follows that

$$
0 \leq \rho\left(x_{n}, \bar{x}\right) \leq \varphi\left(x_{n}\right)-\varphi(\bar{x}),
$$

for all $n \in \mathbb{N}_{0}:=\mathbb{N} \cup\{0\}$.

Consequently,

$$
\alpha_{n} \leq \varphi(\bar{x}) \leq \varphi\left(x_{n}\right),
$$

for all $n \in \mathbb{N}$, yielding for $n \rightarrow \infty, \varphi(\bar{x})=\bar{\alpha}$. But then, the inequalities (4.28) imply $\lim _{n} \rho\left(x_{n}, \bar{x}\right)=0$, that is the sequence $\left(x_{n}\right)$ is $\bar{\rho}$-convergent to $\bar{x}$.

We show now that the conditions (iii) from (4.26) are satisfied.

The relations $\bar{y} \in S(\bar{x}) \subseteq S\left(x_{n}\right)$ imply $\alpha_{n} \leq \varphi(\bar{y}) \leq \varphi(\bar{x})=\bar{\alpha}, \forall n \in \mathbb{N}_{0}$. Letting $n \rightarrow \infty$, one obtains $\varphi(\bar{y})=\bar{\alpha}=\varphi(\bar{x})$.

Also

$$
\bar{y} \in S(\bar{x}) \Longleftrightarrow 0 \leq \rho(\bar{x}, \bar{y}) \leq \varphi(\bar{x})-\varphi(\bar{y})=0,
$$

so that $\rho(\bar{x}, \bar{y})=0$.

Finally, if $z \in S(\bar{y}) \subseteq S(\bar{x})$, then, as above, it follows $\varphi(z)=\varphi(\bar{x})=\varphi(\bar{y})$ and $0 \leq$ $\rho(\bar{y}, z) \leq \varphi(\bar{y})-\varphi(z)=0$, so that $\rho(\bar{y}, z)=0$, that is $z \in \overline{\{\bar{y}\}}$.

The condition (i) is equivalent to $\bar{y} \in S\left(x_{0}\right)$ which is true because $\bar{y} \in S(\bar{x}) \subseteq S\left(x_{0}\right)$.

Condition (ii) follows from the definition of the set $S(\bar{y})$.

Case II. Suppose that, for some $n_{0} \in \mathbb{N}, \varphi\left(x_{n_{0}}\right)=\alpha_{n_{0}}=\inf \varphi\left(S\left(x_{n_{0}}\right)\right)$. Then $x_{n_{0}+1}=$ $x_{n_{0}}$ and, by induction, $x_{n_{0}+k}=x_{n_{0}}$ for all $k \in \mathbb{N}$. 
Then the sequence $\left(x_{n}\right)$ is left $\rho$-K-Cauchy and $\bar{\rho}$-convergent to $x_{n_{0}}$. Also, for $x \in$ $S\left(x_{n_{0}}\right), \varphi(x) \geq \alpha_{n_{0}}=\varphi\left(x_{n_{0}}\right)$, so that the inequalities

$$
0 \leq \rho\left(x_{n_{0}}, x\right) \leq \varphi\left(x_{n_{0}}\right)-\varphi(x) \leq 0,
$$

imply $\rho\left(x_{n_{0}}, x\right)=0$ and $\varphi\left(x_{n_{0}}\right)=\varphi(x)$. It follows also that $x \in \overline{\left\{x_{n_{0}}\right\}} \bar{\rho}$, that is $S\left(x_{n_{0}}\right) \subseteq$ $\overline{\left\{x_{n_{0}}\right\}} \bar{\rho}$.

These show that the condition (iii) is satisfied. The validity of the conditions (i) and (ii) follows as in Case I.

Remark 4.21. Under the hypotheses of Theorem 4.20 it follows

(iii') $\varphi(\bar{y}) \leq \varphi(x)$ for all $x \in \overline{\{\bar{y}}^{\bar{\rho}}$.

Similar conditions appear in Theorem 4.15 too.

The inequality from (ii') follows from the inclusion $S_{\lambda, \rho}(\bar{y}) \subseteq \overline{\{\bar{y}}^{\bar{\rho}}$.

To prove (iii'), observe that $x \in \overline{\{\bar{y}}^{\bar{\rho}} \Longleftrightarrow \rho(\bar{y}, x)=0$. If $x \in S_{\lambda, \rho}(\bar{y})$, then as it is shown in the proof of Theorem 4.20, $\varphi(x)=\varphi\left(y_{*}\right)=\varphi\left(x_{*}\right)$. If $x \in \overline{\{\bar{y}\}} \bar{\rho} \backslash S_{\lambda, \rho}(\bar{y})$, then, by (ii),

$$
\varphi\left(y_{*}\right)<\varphi(x)+\lambda \rho\left(y_{*}, x\right)=\varphi(x) .
$$

To obtain a characterization of completeness, one needs a weaker notion of lower semicontinuity.

Definition 4.22. A function $\varphi: X \rightarrow \mathbb{R} \cup\{\infty\}$, where $(X, \rho)$ is a quasi-semimetric space, is called strictly-decreasing- $\rho$-lsc if for every $\rho$-convergent sequence $\left(x_{n}\right)$ in $X$ such that the sequence $\left(\varphi\left(x_{n}\right)\right)$ is strictly decreasing one has

$$
\varphi(y) \leq \lim _{n} \varphi\left(x_{n}\right)
$$

for every $\rho$-limit $y$ of the sequence $\left(x_{n}\right)$. A sequence $\left(x_{n}\right)$ such that the sequence $\left(\varphi\left(x_{n}\right)\right)$ is strictly decreasing is called strictly $\varphi$-decreasing.

Remark 4.23. In [30] it is shown, by an example, that this notion is strictly weaker than that of $\rho$-lsc, i.e. there exists a function that is strictly-decreasing $\rho$-lsc but not $\rho$-lsc. In fact the everywhere discontinuous function $f(x)=0$ for $x \in \mathbb{Q}$ and $f(x)=1$ for $x \in \mathbb{R} \backslash \mathbb{Q}$, defined on $(\mathbb{R},|\cdot|)$, is strictly-decreasing lsc (because there do not exist a sequence $\left(x_{n}\right)$ in $\mathbb{R}$ such that the sequence $\left(\varphi\left(x_{n}\right)\right)$ be strictly decreasing). The function $f$ is not lsc because $f(x)=1>0=\liminf _{x^{\prime} \rightarrow x} \varphi(x)$ for every $x \in \mathbb{R} \backslash \mathbb{Q}$. Also, it is not usc at every $x \in \mathbb{Q}$.

Remark 4.24. The notion of a function $\varphi: X \rightarrow \mathbb{R} \cup\{\infty\}$, such that $\varphi(x) \leq \lim _{n} \varphi\left(x_{n}\right)$ for every sequence $\left(x_{n}\right)$ in $X$ converging to $x$ such that $\varphi\left(x_{n+1}\right) \leq \varphi\left(x_{n}\right)$ for all $n \in \mathbb{N}$, appears also in the paper [114] of Kirk and Saliga, called lower semicontinuity from above, in connection with Ekeland Variational Principle and Caristi Fixed Point Theorem .

Remark 4.25. In [30] the following sufficient condition for the fulfillment of condition (C2) from Theorem 4.20 was given.

(1) Let $(X, \rho)$ be a quasi-semimetric space and $\varphi: X \rightarrow \mathbb{R} \cup\{+\infty\}$ a proper function. If every sequence $\left(x_{n}\right)$ in the space $(X, \rho)$ such that $\varphi\left(x_{n+1}\right)<\varphi\left(x_{n+1}\right), n \in \mathbb{N}$, and $\sum_{n+1}^{\infty} \rho\left(x_{n}, x_{n+1}\right)<\infty$ is $\bar{\rho}$-convergent to some $x \in X$, and the function $\varphi$ is strictly-decreasing- $\bar{\rho}$-lsc on $\operatorname{dom} \varphi$, then condition $(\mathrm{C} 2)$ is satisfied. 
We mention also the following results relating series completeness and completeness in quasi-metric spaces.

Proposition 4.26 ([30], Proposition 3.10). Let $(X, \rho)$ be a quasi-semimetric space.

(1) If a sequence $\left(x_{n}\right)$ in $X$ satisfies $\sum_{n=1}^{\infty} \rho\left(x_{n}, x_{n+1}\right)<\infty$, then it is left $\rho$-K-Cauchy (or, equivalently, right $\bar{\rho}-K$-Cauchy).

(2) The space $X$ is left $\rho$-K-complete if and only if every sequence $\left(x_{n}\right)$ in $X$ satisfying $\sum_{n=1}^{\infty} \rho\left(x_{n}, x_{n+1}\right)<\infty$ is $\rho$-convergent to some $x \in X$.

(3) The space $X$ is right $\bar{\rho}-K$-complete if and only if every sequence $\left(x_{n}\right)$ in $X$ satisfying $\sum_{n=1}^{\infty} \rho\left(x_{n}, x_{n+1}\right)<\infty$ is $\bar{\rho}$-convergent to some $x \in X$.

Proof. (Sketch) The assertion (1) follows from the triangle inequality and the Cauchy criterion of convergence applied to the series $\sum_{n=1}^{\infty} \rho\left(x_{n}, x_{n+1}\right)$ :

$$
\rho\left(x_{n}, x_{n+k}\right) \leq \sum_{i=0}^{k-1} \rho\left(x_{n+i}, x_{n+i+1}\right)<\varepsilon .
$$

(2) and (3). If $\left(x_{n}\right)$ is left $\rho$ - $K$-Cauchy, then there exist the numbers $n_{1}<n_{2}<\ldots$ such that $\rho\left(x_{n_{k}}, x_{n_{k+1}}\right)<1 / 2^{k}, k \in \mathbb{N}$. Then $\sum_{k=1}^{\infty} \rho\left(x_{n_{k}}, x_{n_{k+1}}\right)<\infty$ so that, by hypothesis, there exists $x \in X$ with $\lim _{k} \rho\left(x, x_{n_{k}}\right)=0\left(\lim _{k} \bar{\rho}\left(x, x_{n_{k}}\right)=0\right)$. By Remarks 4.4. (2) $\lim _{n} \rho\left(x, x_{n}\right)=0\left(\operatorname{resp} \lim _{n} \bar{\rho}\left(x, x_{n}\right)=0\right)$.

We will present now a characterization of completeness of quasi-semimetric spaces in terms of the Ekeland Variational Principle (Theorem 4.20).

Theorem 4.27 (A characterization of completeness). For any quasi-semimetric space $(X, \rho)$ the following conditions are equivalent.

(1) The space $X$ is right $\bar{\rho}$ - $K$-complete.

(2) For every proper, bounded from below, and strictly-decreasing- $\bar{\rho}$-lsc function $\varphi$ : $X \rightarrow \mathbb{R} \cup\{+\infty\}$ and for any $x_{0} \in \operatorname{dom}(\varphi)$ there is $\bar{x} \in X$ such that for every $\bar{y} \in S_{1}(\bar{x})$ one has

(i) $\rho\left(x_{0}, \bar{y}\right) \leq \varphi\left(x_{0}\right)-\varphi(\bar{y})$;

(ii) $\varphi(\bar{y})<\varphi(x)+\rho(\bar{y}, x)$, for all $x \in X \backslash \overline{\{\bar{y}\}}^{\bar{\rho}}$;

(iii) $\varphi(\bar{y}) \leq \varphi(x)$ for all $x \in \overline{\{\bar{y}\}}^{\bar{\rho}}$.

Proof. (1) $\Longrightarrow(2)$ This implication follows by Theorem 4.20 (see also Proposition 4.26 and the Remarks 4.21, 4.251).

The proof of the implication $(2) \Longrightarrow(1)$ is similar to that of the implication $(3) \Longrightarrow(1)$ in Theorem 4.15, working with the conjugate metric $\bar{\rho}$ instead of $\rho$.

The following example shows that the completeness could not hold if we suppose that only the conditions (i) and (ii) from Theorem 4.27 hold.

Example 4.28. Let $x_{n}=-n, n \in \mathbb{N}_{0}$, and $X=\left\{x_{n}: n \in \mathbb{N}_{0}\right\}$ with the metric $q\left(x_{n}, x_{m}\right)=\left(x_{m}-x_{n}\right)^{+}=(-m+n)^{+}=n-m$ if $n>m$ and $=0$ if $n \leq m$ (see Example 4.2). Then $q\left(x_{n}, x_{n+1}\right)=0$ for all $n \in \mathbb{N}_{0}$ and the space $X$ is not right $\bar{q}$ - $K$-complete (see Example 4.6). Let $\varphi: X \rightarrow[0, \infty)$ be an arbitrary function. For $\bar{x}=x_{0}$,

$$
\varphi(\bar{x})=\varphi\left(x_{0}\right) \leq \varphi\left(x_{0}\right)=\varphi\left(x_{0}\right)+q\left(\bar{x}, x_{0}\right) .
$$

Since $q\left(x_{0}, x_{n}\right)=0$ for all $n \in \mathbb{N}_{0}$ the condition

$$
\varphi\left(x_{0}\right)<\varphi\left(x_{n}\right)+q\left(x_{0}, x_{n}\right) \text { for all } n \in \mathbb{N}_{0} \text { with } q\left(x_{0}, x_{n}\right)>0,
$$


is trivially satisfied.

Remark 4.29. Bao and Soubeyran use the notions of forward and backward in a quasisemimetric space $(X, \rho)$, where forward means with respect to $\bar{\rho}$, while backward means with respect to $\rho$.

For instance, a sequence $\left(x_{n}\right)$ in $X$ is:

- forward convergent to $x$ if $\quad \bar{\rho}\left(x, x_{n}\right)=\rho\left(x_{n}, x\right) \rightarrow 0$, i.e. it is $\bar{\rho}$-convergent to $x$;

- forward Cauchy if for every $\varepsilon>0$ there exists $n_{\varepsilon} \in \mathbb{N}$ such that $\rho\left(x_{n}, x_{n+k}\right)<\varepsilon$, for all $n \geq n_{\varepsilon}$ and all $k \in \mathbb{N}$, i.e. if it is left $\rho$-K-Cauchy, or equivalently, right $\bar{\rho}-K$-Cauchy.

- backward convergent to $x$ if $\rho\left(x, x_{n}\right) \rightarrow 0$, i.e. it is $\rho$-convergent to $x$;

- backward Cauchy if for every $\varepsilon>0$ there exists $n_{\varepsilon} \in \mathbb{N}$ such that $\rho\left(x_{n+k}, x_{n}\right)<\varepsilon$, for all $n \geq n_{\varepsilon}$ and all $k \in \mathbb{N}$, i.e. if it is right $\rho$-K-Cauchy, or equivalently, left $\bar{\rho}$ - $K$-Cauchy.

The space $X$ is called forward-forward complete if every forward Cauchy sequence is forward convergent, i.e. if it is right $\bar{\rho}-K$-complete.

The backward notion of completeness is defined on an analogous way: the quasi-metric space $(X, \rho)$ is backward-backward complete if every backward Cauchy sequence is backward convergent, i.e. if it is right $\rho$ - $K$-complete. One can define also combined notions of completeness: backward-forward (meaning left $\bar{\rho}$ - $K$-completeness) and forward-backward (meaning left $\rho$ - $K$-completeness).

Bao, Soubeyran and Mordukhovich applied systematically variational principles in quasimetric spaces to various domains of human knowledge as psychology in [31, to capability of wellbeing and rationality in [32], and to group dynamics in [33].

\section{TOPOLOGY AND ORDER}

In this section we shall discuss some results relating topology and order.

5.1. Partially ordered sets. Let $X$ be a nonempty set. A preorder on $X$ is a reflexive and transitive relation $\leq \subseteq X^{2}$. The pair $(X, \leq)$ is called a partially preordered set. An antisymmetric preorder is called an order on $X$. The pair $(X, \leq)$ is called a partially ordered set, or a poset in short.

Let $(X, \leq)$ be a partially preordered set. For a nonempty subset $A$ of a partially preordered set $(X, \leq)$ one puts

$$
\uparrow A:=\{y \in X: \exists x \in A, x \leq y\} \text { and } \downarrow A:=\{y \in X: \exists x \in A, y \leq x\} .
$$

In particular, for $x \in X$,

$$
\uparrow x:=\uparrow\{x\} \text { and } \downarrow x=\downarrow\{x\} .
$$

The set $A$ is called upward closed if $A=\uparrow A$ and downward closed if $A=\downarrow A$.

A nonempty subset $A$ of $X$ is called totally ordered (or a chain) if any two elements of $A$ are comparable.

A nonempty subset $D$ of $X$ is called directed if for any two elements of $x, y \in A$ there exists $z \in A$ such that $x \leq z$ and $y \leq z$.

Let $A \subseteq X$. Then

- an upper bound for $A$ is an element $z \in X$ such that $\forall x \in A, x \leq z$;

- if $z$ is an upper bound for $A$ and $z \in A$ then $z$ is the greatest element of $A$;

- the set $A$ is called upper-bounded if it has at least one upper bound; 
- the least upper bound of $A$ is called the supremum of $A$, $\operatorname{denoted}$ by $\sup A$ (or by $\bigvee A)$;

- in the case of two elements $x, y \in X$ one uses the notation $x \vee y:=\bigvee\{x, y\}$;

- the greatest element of $X$ is called unity and is denoted by $\top$ (or by 1); the least element of $X$ is called zero and is denoted by $\perp$ (or by 0 );

Dually, one defines lower bounds, infima, etc. (we have used the notion of least element yet). The infimum is denoted by $\inf A$ (or by $\bigwedge A$ ). Also $x \wedge y:=\bigwedge\{x, y\}$.

Definition 5.1. A poset $(X, \leq)$ is called

- an upper semi-lattice if every two elements $x, y \in X$ have a sup, $x \vee y$;

- an lower semi-lattice if every two elements $x, y \in X$ have an inf, $x \wedge y$;

- if $(X, \leq)$ is an upper semi-lattice and a lower semi-lattice, then it is called a lattice, that is for every $x, y \in X$ there exist $x \wedge y$ and $x \vee y$;

- a lattice $(X, \leq)$ is called complete if for every subset $A$ of $X$ there exist $\sup A$ and $\inf A$.

- a poset $(X, \leq)$ is called directed (chain, boundedly) complete if every directed (totally ordered, upper bounded) subset of $X$ has supremum. A directed complete partially ordered set is denoted in short by dcpo.

Remarks 5.2. Let $(X, \leq)$ be a poset.

1. If $X$ has a greatest element $\top$, then $\top=\sup X$. If $X$ has a least element $\perp$, then $\perp=\inf X$.

2. In the definition of a complete lattice $(X, \leq)$ it suffices to request that every subset of $X$ has a supremum, because $X$ has a least element $\perp=\sup \emptyset$ and the infimum of a subset $A$ of $X$ is the supremum of the set $L_{A}$ of all lower bounds of $A$ (this set is nonempty because $\perp \in A$ ).

\section{Mappings between partially preordered sets}

Let $(X, \leq),(Y, \preceq)$ be two partially preordered sets. A mapping $f: X \rightarrow Y$ is called

- increasing if $\forall x, y \in X, x \leq y \Rightarrow f(x) \preceq f(y)$;

- decreasing if $\forall x, y \in X, x \leq y \Rightarrow f(y) \preceq f(x)$;

- monotonic if it is increasing or decreasing.

One says that $f$ preserves

- suprema if and only if for every $A \subseteq X$, the existence of $\sup A$ implies the existence of $\sup f(A)$ and the equality $\sup f(A)=f(\sup A)$.

- finite (directed, chain) suprema if and only if the above condition holds for every finite (respectively directed, totally ordered) subset $A$ of $X$.

Similar definitions can be given for infima.

Remark 5.3. A mapping preserving finite suprema is increasing.

Indeed, if $x \leq y$ in $X$, then $y=\sup \{x, y\}$, and so $f(y)=\sup \{f(x), f(y)\}$, implying $f(x) \preceq f(y)$.

5.2. Order relations in topological spaces. The specialization order of a topological space $(X, \tau)$ is the partial order defined by

$$
x \leq_{\tau} y \Longleftrightarrow x \in \overline{\{y\}},
$$

that is $y$ belongs to every open set containing $x$.

Proposition 5.4. Let $(X, \tau)$ be a topological space. The relation defined by (5.2) is a preorder. 
It is an order if and only if $X$ is $T_{0}$.

If $X$ is $T_{1}$, then $\leq_{\tau}$ is the equality relation in $X$.

Proof. Since $x \in \overline{\{x\}}$ it follows $x \leq_{\tau} x$.

The transitivity follows from the following implication

$$
x \in \overline{\{y\}} \text { and }\{y\} \subseteq \overline{\{z\}} \Rightarrow x \in \overline{\{y\}} \subseteq \overline{\overline{\{z\}}}=\overline{\{z\}},
$$

that is

$$
x \leq_{\tau} y \text { and } y \leq_{\tau} z \Rightarrow x \leq_{\tau} z .
$$

Suppose that $X$ is $T_{0}$ and $x, y$ are two distinct points in $X$. Then there exists an open set $V$ that contains exactly one of this points. If $x \in V$ and $y \notin V$, then $x \notin \overline{\{y\}}$, that is the relation $x \leq_{\tau} y$ does not hold. If $y \in V$ and $x \notin V$, then $y \notin \overline{\{x\}}$, that is the relation $y \leq_{\tau} x$ does not hold. This means that we can not have simultaneously $x \leq_{\tau} y$ and $y \leq_{\tau} x$ for a pair of distinct elements in $X$.

Similar reasonings show that $X$ is $T_{0}$ if $\leq_{\tau}$ is a partial order (i.e. it is antisymmetric).

The topological space $X$ is $T_{1}$ if and only if $\overline{\{x\}}=\{x\}$ for every $x \in X$. Consequently,

$$
x \leq_{\tau} y \Longleftrightarrow x \in \overline{\{y\}}=\{y\} \Longleftrightarrow x=y .
$$

One shows also that if the order relation $\leq_{\tau}$ is equality, then $X$ is $T_{1}$.

In the following results the order notions are considered with respect to the order $\leq_{\tau}$.

Proposition 5.5. Let $(X, \tau)$ be a topological space and $A \subseteq X$.

(1) If the set $A$ is open, then it is upward closed, i.e. $\uparrow A=A$.

(2) If the set $A$ is closed, then it is downward closed, i.e. $\downarrow A=A$.

Proof. (1) It is a direct consequence of definitions. Let $x \in A$ and $y \in X, x \leq_{\tau} y$. Since $A$ is open, this inequality implies $y \in A$.

(2) Let $x \in A$ and $y \in X, y \leq_{\tau} x$. Then $y \in \overline{\{x\}} \subseteq \bar{A}=A$.

Let us define the saturation of a subset $A$ of $X$ as the intersection of all open subsets of $X$ containing $A$. The set $A$ is called saturated if equals its saturation.

Proposition 5.6. Let $(X, \tau)$ be a topological space.

(1) For every $x \in X, \downarrow x=\overline{\{x\}}$.

(2) For any subset $A$ of $X$ the saturation of $A$ coincides with $\uparrow A$.

Proof. (1) This follows from the equivalence

$$
y \leq_{\tau} x \Longleftrightarrow y \in \overline{\{x\}} .
$$

(2) Since every open set is upward closed, $U \in \tau$ and $U \supset A$ implies $U \supset \uparrow A$, that is

$$
\uparrow A \subseteq \bigcap\{U \in \tau: A \subseteq U\} .
$$

If $y \notin \uparrow A$, then for every $x \in A$ there exists $U_{x} \in \tau$ such that $x \in U_{x}$ and $y \notin U_{x}$. It follows $y \notin V:=\bigcup\left\{U_{x}: x \in A\right\} \in \tau$ and $A \subseteq V$, hence $y \notin \bigcap\{U \in \tau: A \subseteq U\}$, showing that

$$
\complement(\uparrow A) \subseteq \complement(\bigcap\{U \in \tau: A \subseteq U\}) \Longleftrightarrow \bigcap\{U \in \tau: A \subseteq U\} \subseteq \uparrow A
$$

\section{Compactness}

We present following [75, p. 69] a result on compactness. 
Proposition 5.7. Let $(X, \tau)$ be a topological space. A subset $K$ of $X$ is compact if and only if its saturation $\uparrow K$ is compact.

Proof. The equivalence follows from the following remark: since every open subset of $X$ is upward closed with respect to the specialization order, the following equivalence is true

$$
K \subseteq \bigcup\left\{U_{i}: i \in I\right\} \Longleftrightarrow \uparrow K \subseteq \bigcup\left\{U_{i}: i \in I\right\},
$$

for every family $\left\{U_{i}: i \in I\right\} \subseteq \tau$.

An order $\preceq$ on a topological space $(X, \tau)$ is said to be closed if and only if its graph $\operatorname{Graph}(\preceq):=\{(x, y) \in X \times X: x \preceq y\}$ is closed in $X \times X$ with respect to the product topology. The existence of a closed order on a topological space forces the topology to be Hausdorff.

Proposition 5.8 ([75, P.3.9.12). If on a topological space $(X, \tau)$ there exists a closed order $\preceq$, then the topology $\tau$ is Hausdorff.

Proof. Let $x, y$ be distinct points in $X$. Then the relations $x \preceq y$ and $y \preceq x$ can not both hold. Suppose, without the loose of generality, that $x \preceq y$ does not hold, that is $(x, y) \notin \operatorname{Graph}(\preceq)$. Then there exists the open neighborhoods $U, V$ of $x$ and $y$, respectively, such that $(U \times V) \cap \operatorname{Graph}(\preceq)=\emptyset$. The proof will be done if we show that $U \cap V=\emptyset$. Indeed supposing that there exists $z \in W:=U \cap V$, one obtains the contradiction

$$
(z, z) \in(W \times W) \cap \operatorname{Graph}(\preceq) \subseteq(U \times V) \cap \operatorname{Graph}(\preceq)=\emptyset .
$$

For other properties of topological spaces endowed with a closed order (e.g. compactness), see [75, Section 9.1.1]

5.3. Topologies on ordered sets - Alexandrov's, the upper topology, Scott's, the interval topology. Consider a partially preordered set $(X, \leq)$. We are interested to define a topology $\tau$ on $X$ such that the specialization preordering $\leq_{\tau}$ coincides with $\leq$. The answer is, in general no. For instance, on $\mathbb{R}$, with the usual ordering, we can consider several topologies (the usual, the discrete, etc), all having as specialization preordering the equality.

Let $(X, \leq)$ be a partially preordered set. We shall consider three topologies on $X$ such that the corresponding specialization preorderings coincide with $\leq$, as well as the interval topology and the Moore-Smith order topology.

\section{The Alexandrov topology - the finest}

This is the finest of these topologies.

Proposition 5.9 (Alexandov topology). Let $(X, \leq)$ be a partially preordered set. Then there exists a finest topology $\tau_{a}$ on $X$, called the Alexandrov topology, such that the specialization preordering $\leq_{\tau_{a}}$ coincides with $\leq$. This topology is characterized by the condition

(i) the open sets are exactly the upward closed sets,

or, equivalently,

(ii) the closed sets are exactly the downward closed sets.

In this case, $\uparrow x \in \tau_{a}$ for every $x \in X$.

Proof. It is clear that the upward closed subsets of $X$ form a topology $\tau_{a}$ on $X$. Since $\uparrow x$ is upward closed, it follows $\uparrow x \in \tau_{a}$. 
We have

$$
\begin{aligned}
x \leq_{a} y & \Longleftrightarrow x \in \operatorname{cl}_{\tau_{a}}\{y\} \\
& \Longleftrightarrow \forall Z \in \tau_{a}, x \in Z \Rightarrow y \in Z .
\end{aligned}
$$

If $x \leq_{a} y$, then $x \in \uparrow x$ and $\uparrow x \in \tau_{a}$, imply $y \in \uparrow x$, that is, $x \leq y$.

If $x \leq y$ and $Z \in \tau_{a}$ contains $x$, then $y \in Z$, as $Z$ is upward closed, showing that $x \leq_{a} y$.

We use the notation $X_{a}$ for $\left(X, \tau_{a}\right)$.

Remark 5.10. Since for every upward closed subset $Z$ of $X, Z=\bigcup\{\uparrow z: z \in Z\}$ it follows that the Alexandrov topology $\tau_{a}$ is generated by the family of sets $\{\uparrow x: x \in X\}$.

The upper topology - the coarsest

Proposition 5.11. Let $(X, \leq)$ be a partially preordered set. Then there exists a coarsest topology $\tau_{u}$ on $X$ such that the specialization preordering $\leq_{\tau_{u}}$ coincides with $\leq$.

$A$ subbase of $\tau_{u}$ is formed by the complements of the sets $\downarrow x, x \in X$.

$A$ basis of $\tau_{u}$ is formed by the complements of the sets $\downarrow E$ for $E \subseteq X, E$ finite.

Proof. It is easy to check that the sets $\downarrow E$ for $E \subseteq X, E$ finite, form a basis of a topology $\tau_{u}$ on $X$. Denote by $\leq_{u}$ the specialization order determined by this topology.

Let $x \leq_{u} y$. By definition this is equivalent to $x \in \overline{\{y\}}$. But

$$
\begin{aligned}
x \in \overline{\{y\}} & \Longleftrightarrow \forall z,[x \in X \backslash \downarrow z \Rightarrow y \in X \backslash \downarrow z] \\
& \Longleftrightarrow \forall z,[y \in \downarrow z \Rightarrow x \in \downarrow z] \\
& \underset{(z=y)}{\Longrightarrow} x \in \downarrow y \Longleftrightarrow x \leq y .
\end{aligned}
$$

Conversely, suppose $x \leq y$.

If for some $z \in X, y \in \downarrow z$, then $x \leq y$ and $y \leq z$ would imply $x \leq z$, that is $x \in \downarrow z$. Consequently

$$
x \in X \backslash \downarrow z \Rightarrow y \in X \backslash \downarrow z,
$$

for all $z \in X$, which is equivalent to $x \leq_{u} y$.

\section{The Scott topology}

This is a topology between $\tau_{u}$ and $\tau_{a}$. It is defined in the following way.

Let $(X, \leq)$ be a partially ordered set. A subset $U$ of $X$ is $S$ cott open if and only if the following two conditions hold:

(i) $U$ is upward closed, and

(ii) for every nonempty directed subset $D$ of $X \operatorname{such}$ that $\sup D$ exists (in $X$ ) and belongs to $U$, there exists $d \in D$ such that $d \in U$.

Proposition 5.12. Let $(X, \leq)$ be a partially ordered set.

(1) The family of Scott open subsets of $X$ forms a topology denoted by $\tau_{\sigma}$.

(2) A subset $F$ of $X$ is Scott closed if and only if the following two conditions hold:

(i) $F$ is downward closed, and

(ii) for every nonempty directed subset $D$ of $F$ if $\sup D$ exists (in $X$ ), then $\sup D \in F$.

In particular, the set $\downarrow y$ is Scott closed for every $y \in Y$.

(3) The specialization order corresponding to $\tau_{\sigma}$ agrees with $\leq$ and

$$
\tau_{u} \leq \tau_{\sigma} \leq \tau
$$


(4) Let $(X, \tau)$ be a topological space, $\leq_{\tau}$ the specialization order corresponding to $\tau$ and $\sigma=\sigma\left(\leq_{\tau}\right)$ the Scott topology corresponding to $\leq_{\tau}$. Then the set $\overline{\{x\}}^{\tau}$ is Scott closed (i.e $\sigma$-closed) for every $x \in X$.

Proof. (1) Let $U_{i}, i=1, \ldots, n$, be Scott open sets. Then $U:=\bigcap\left\{U_{i}: 1 \leq i \leq n\right\}$ is upward closed. Suppose that $D$ is a directed set in $X$ such that $\sup D$ exists and belongs to $U$. Then $\sup D \in U_{i}$ implies the existence of $x_{i} \in D \bigcap U_{i}, i=1, \ldots, n$. Since $D$ is directed there exists $x \in D$ with $x_{i} \leq x, i=1, \ldots, n$. Since each $U_{i}$ is upward closed, $x \in U_{i}, i=1, \ldots, n$, that is $x \in U$, showing that $U$ is Scott open too. It easy to show that the union of an arbitrary family of Scott open sets is again Scott open.

The proof of (2) follows from the equality $F=X \backslash U$ relating open sets $U$ and closed sets $F$.

To prove that the set $\downarrow y$ is Scott closed, let $D \subseteq \downarrow y$ be a directed set and $d_{0}=\sup D$. Since, $d \leq y$ for all $d \in D$, it follows $d_{0} \leq y$, i.e. $d_{0} \in \downarrow y$, showing that $\downarrow y$ is Scott closed.

(3) Denote by $\leq_{\sigma}$ the specialization order corresponding to $\tau_{\sigma}$ and let $x \leq y$. If $U$ is Scott open and $x \in U$, then $y \in U$, as $U$ is upward closed. Consequently $x \leq_{\sigma} y$.

Suppose now $x \leq_{\sigma} y$. The set $\downarrow y$ is Scott closed. If $x$ would belong to $X \backslash \downarrow y$, then $y \in X \backslash \downarrow y$, a contradiction, so $x$ must belong to $\downarrow y$, that is $x \leq y$.

(4) The proof is based on (2). Denoting by $\leq_{\tau}, \leq_{\sigma}$ the specialization orders corresponding to $\tau$ and $\sigma$, respectively, then $\leq_{\tau}=\leq_{\sigma}$.

Show first that the set $\overline{\{x\}}^{\tau}$ is $\leq_{\tau}$-downward closed.

Indeed, let $y \in \overline{\{x\}}^{\tau}$ and $y^{\prime} \leq_{\tau} y$. Then $y \leq_{\tau} x$ and $y^{\prime} \leq_{\tau} y$ imply $y^{\prime} \leq_{\tau} x$, that is $y^{\prime} \in \overline{\{x\}}^{\tau}$.

Let us verify condition (ii) from (2).

If $\left\{x_{i}: i \in I\right\}$ is a directed set contained in $\overline{\{x\}}^{\tau}$, then $x_{i} \leq_{\tau} x$ for all $i \in I$, and so $\sup _{i} x_{i} \leq_{\tau} x$, or equivalently, $\sup _{i} x_{i} \in \overline{\{x\}}^{\tau}$.

In the following proposition we characterize the continuity with respect to the Scott topology. We use the notation $X_{\sigma}$ for $\left(X, \tau_{\sigma}\right)$.

Proposition 5.13. Let $X, Y$ be a partially ordered sets and $f: X \rightarrow Y$. The following are equivalent.

(1) The function $f$ is continuous with respect to the Scott topologies on $X$ and $Y$, respectively.

(2) The function $f$ satisfies the following conditions:

(i) $f$ is increasing;

(ii) $f$ preserves the suprema of directed sets.

Proof. All closures that appear in this proof are considered with respect to the Scott topology.

$(2) \Rightarrow(1)$. The continuity of $f$ is equivalent to each of the following conditions

(i) $\quad f^{-1}(Z)$ is closed for every closed subset $Z$ of $Y$;

$$
f(\bar{A}) \subseteq \overline{f(A)} \text { for every subset } A \text { of } X .
$$

Let $Z \subseteq Y$ be Scott closed. We shall use Proposition [5.12. (2) to show that $f^{-1}(Z)$ is Scott closed.

If $x \in f^{-1}(Z)$ and $x^{\prime} \leq x$, then $f(x) \in Z$ and $f\left(x^{\prime}\right) \leq f(x)$. Since $Z$ is downward closed it follows $f\left(x^{\prime}\right) \in Z$, which is equivalent to $x^{\prime} \in f^{-1}(Z)$. Consequently $f^{-1}(Z)$ is downward closed. 
Let now $\left(x_{i}\right)_{i \in I}$ be a directed set contained in $f^{-1}(Z)$ such that $x=\sup _{i} x_{i}$ exists. Then $\left(f\left(x_{i}\right)\right)_{i \in I}$ is a directed set in $Y$ contained in $Z$. By hypothesis $f(x)=\sup _{i} f\left(x_{i}\right)$ and, since $Z$ is Scott closed, $f(x) \in Z$ which is equivalent to $x \in f^{-1}(Z)$.

$(1) \Rightarrow(2)$. Suppose that $f$ is continuous with respect to the Scott topologies on $X$ and $Y$, respectively.

Let $x^{\prime} \leq x$ in $X$. Taking into account the continuity of $f$ we have

$$
x^{\prime} \leq x \Longleftrightarrow x^{\prime} \in \overline{\{x\}} \Rightarrow f\left(x^{\prime}\right) \in f(\overline{\{x\}}) \subseteq \overline{f(x)},
$$

which shows that $f\left(x^{\prime}\right) \leq f(x)$ in $Y$.

Let now $\left(x_{i}\right)_{i \in I}$ be a directed set in $X$ such that $x=\sup _{i} x_{i}$ exists. Since $f$ is increasing, it follows $f\left(x_{i}\right) \leq f(x)$ for all $i \in I$. Let $y \in Y$ be such that $f\left(x_{i}\right) \leq y$ for all $i \in I$. Then

$$
\forall i, \quad f\left(x_{i}\right) \leq y \Longleftrightarrow \forall i, \quad f\left(x_{i}\right) \in \overline{\{y\}} \Longleftrightarrow \forall i, \quad x_{i} \in f^{-1}(\overline{\{y\}}) .
$$

Since $f^{-1}(\overline{\{y\}})$ is Scott closed, it follows $x=\sup _{i} x_{i} \in f^{-1}(\overline{\{y\}})$, which implies $f(x) \in$ $\overline{\{y\}}$, that is $f(x) \leq y$. Consequently, $f(x)$ is the least upper bound of $\left(f\left(x_{i}\right)\right)_{i \in I}$.

Remark 5.14. A mapping satisfying condition (i) and (ii) from Proposition 5.13 is called Scott continuous. In fact, by Remark 5.3, it suffices to suppose that $f$ satisfies only the condition (ii).

Example 5.15 ([75]). A subset of $\mathbb{R}$ is compact and saturated with respect to the Scott topology if and only if it is the empty set or of the form $[\alpha, \infty)$ for some $\alpha \in \mathbb{R}$.

\section{The interval topology and the Moore-Smith order topology}

These topologies were defined by Frink [69]. By a closed interval in a poset $(X, \leq)$ one understands a set of the form

$$
\begin{aligned}
& \uparrow a=\{x \in X: a \leq x\}, \quad \downarrow b=\{y \in X: y \leq b\}, \quad \text { or } \\
& {[a, b]=\{x \in X: a \leq x \leq b\}=\uparrow a \cap \downarrow b,}
\end{aligned}
$$

for $a, b \in X$. By definition, a subset $Y$ of $X$ is closed with respect to the interval topology if it can be written as the intersection of finite unions of sets of the form (5.4). It is shown in [69] that the family $\mathcal{F}_{\leq}$of closed sets defined above satisfies the axioms of closed sets:

$$
\begin{aligned}
& \text { (i) } \emptyset, X \in \mathcal{F}_{\leq} ; \quad \text { (ii) } F_{i} \in \mathcal{F}_{\leq}, i \in I, \Rightarrow \bigcap_{i \in I} F_{i} \in \mathcal{F}_{\leq} \text {; } \\
& \text { (iii) } F_{1}, F_{2} \in \mathcal{F}_{\leq} \Rightarrow F_{1} \cup F_{2} \in \mathcal{F}_{\leq} .
\end{aligned}
$$

If the set $X$ is totally ordered (i.e. it is a chain), then the interval topology defined above coincides with the intrinsic topology, which is the topology having as basis of open sets the intervals

$$
(a, b):=\{x \in X: a<x<b\},
$$

for $a, b \in X$ (see [69, Th. 3]). (Recall that we write $x<y$ for " $x \leq y$ and $x \neq y$ ").

Remark 5.16. By analogy with the upper topology one can define the lower topology $\tau_{l}$ as that generated by the basis formed of the complements of the sets $\uparrow E$ for $E \subseteq X, E$ finite. The interval topology $\tau_{\leq}$is the supremum of these two topologies: $\tau_{\leq}=\tau_{u} \vee \tau_{l}$.

We mention also the following result.

Theorem 5.17 ([69], Th. 9). Every complete lattice is compact in its interval topology. 
Proof. We include the simple proof of this result. Let $(X, \leq)$ be a complete lattice with 0 the least and 1 the greatest element. Then $\uparrow a=[a, 1]$ and $\downarrow b=[0, b]$, so that the intervals $[a, b], a, b \in X$, form a subbase of the interval topology. By Alexander subbase theorem ([109, p. 139] it is sufficient to show that every family $\left[a_{i}, b_{i}\right], i \in I$, of intervals in $X$ having the finite intersection property has nonempty intersection. Since $\left[a_{i}, b_{i}\right] \cap\left[a_{j}, b_{j}\right] \neq$ $\emptyset \Longleftrightarrow a_{i} \vee a_{j} \leq b_{i} \wedge b_{j}$, it follows $a_{i} \leq b_{j}$ for all $i, j \in I$. Hence

$$
a:=\sup _{i \in I} a_{i} \leq \inf _{j \in I} b_{j}=: b,
$$

and $\emptyset \neq[a, b] \subseteq \bigcap_{i \in I}\left[a_{i}, b_{i}\right]$.

Frink 69] considered also the Moore-Smith order topology defined in the following way. A net $\left(x_{i}: i \in I\right)$ in a poset $(X, \leq)$ is said to converge to $x \in X$ if there exist an increasing net $\left(u_{i}: i \in I\right)$ and a decreasing one $\left(v_{i}: i \in I\right)$ such that

(i) $u_{i} \leq x_{i} \leq v_{i}$ for all $i \in I$ and

(ii) $\sup _{i} u_{i}=x=\inf _{i} v_{i}$.

By definition, an element $x \in X$ belongs to the closure $\bar{Y}$ of a subset $Y$ of $X$ if and only if there exists a net in $Y$ that converges to $x$. This closure operation satisfies the conditions
(a) $\bar{\emptyset}=\emptyset$;
(b) $Y \subseteq \bar{Y}$;
(c) $\overline{Y_{1} \cup Y_{2}}=\overline{Y_{1}} \cup \overline{Y_{2}}$,

for all $Y, Y_{1}, Y_{2} \subseteq X$, but not the condition $\overline{\bar{Y}}=\bar{Y}$, so it does not generate a topology, see Kelley [109, p. 43]. In spite of this fact we call it the Moore-Smith order topology. If $(X, \leq)$ is totally ordered, then it agrees with the interval topology [69, Th. 3]. If $(X, \leq)$ is a distributive lattice, then the lattice operations $\vee$ and $\wedge$ are continuous with respect to the Moore-Smith order topology [69, Th. 2].

Remark 5.18. Motivated by applications to computer science, mainly to denotational semantics of functional programming languages, topological and categorical methods applied to partially ordered sets were developed. A branch of this is known under the name of continuous lattices, whose study was initiated by Dana Scott [181 in 1971. Roughly speaking these are complete lattices $(X, \leq)$ with Scott continuous meet and join operations, which means that

$$
x \wedge \sup D=\sup \{x \wedge d: d \in D\} \quad \text { and } \quad x \vee \inf D=\inf \{x \vee d: d \in D\},
$$

for every nonempty directed subset $D$ of $X$.

Another one is the so called domain theory. Essentially it is concerned with the study of lattices or of directed complete partially ordered sets (known as dcpo, see Definition 5.1) equipped with a $T_{0}$ topology compatible with the order. A good introduction to this area is given in the book [75] (which we have partially followed in our presentation), and in the paper [2]. For a comprehensive presentation we recommend the monograph [73], see also [187. Notice also that a functional analysis within the context of $T_{0}$ topology was recently developed, see for instance [107, 108. It turned out that a lot of results from Hausdorff functional analysis (Hausdorff topological vector, Hausdorff locally convex spaces and Banach spaces) have their analogues in some algebraic structures - vector spaces, cones, universal algebras, etc - equipped with a compatible $T_{0}$ topology. 


\section{FiXed Points in PARTially ORDERED SETS}

In this section we shall present some fixed point results in partially ordered sets and their impact on the completeness of the underlying ordered set.

6.1. Fixed point theorems. These fixed point theorems bear different names in different publications. The explication is that many mathematicians contributes to their final shape, and the authors choose one, or several of them, to name a theorem.

Recall that "poset" is a short-hand for "partially ordered set".

Theorem 6.1 (Zermelo). Let $(X, \leq)$ be a chain-complete poset and $f: X \rightarrow X$ a mapping such that $x \leq f(x)$ for all $x \in X$.

Then $f$ has a fixed point. More precisely, for every $x \in X f$ has a fixed point $y$ above $x$ (i.e. $f(y)=y$ and $x \leq y$ ).

If, further, $f$ is increasing, then, for every $x \in X, f$ has a least fixed point above $x$.

A mapping $f: X \rightarrow X$ satisfying $x \leq f(x)$ for all $x \in X$ is called progressive in [94], inflationary in [75], extensive in [119].

This theorem is attributed to Bourbaki-Witt in [75] (with reference to Bourbaki [40] and Witt [217]), to Bourbaki-Kneser in [221]. As it follows from the discussion about this matter in the survey paper by Jachymski [94], who proposed the name Zermelo FPT, this fixed point theorem appears only implicitly in Zermelo's papers on well-ordering (from 1904 and 1908), and it was put in evidence later. Accepting this principle (equivalent to the Axiom of Choice (AC)), the proof is immediate, but there are proofs independent of (AC), see [94]. A brief historical survey is given also in Blanqui [38. We shall not enter into this delicate question of whether a specific result depends or not of the (AC). An exhaustive treatment is given in the monographs [84 and [173]. Concerning its relevance for fixed points we recommend the papers by Tasković [207, 208, 209] and Mańka [134, 135, 136. Among other things, Mańka has found a proof of Caristi's fixed point theorem, independent of the (AC).

Remark 6.2. In Bourbaki [40] Zermelo FPT is formulated for a poset in which every wellordered subset has a supremum, an apparently stronger form. But as it was shown by Markowski [138] these conditions are equivalent: a poset $X$ is chain complete if and only if every well-ordered subset of $X$ has a supremum. In fact, according to the comments before Lemma 1.4 in [185], this result can be considered as a part of the folklore, the essential part of the proof - that every chain contains a well-ordered cofinal subset - appears as exercises in Halmos' Naive set theory, and in Birkhoff's Lattice theory.

Another important result is the following one.

Theorem 6.3 (Knaster-Tarski). Let $(X, \leq)$ be a poset and $f: X \rightarrow X$ an increasing function. If

(i) there exists $z \in X$ such that $z \leq f(z)$, and

(ii) every chain in $\uparrow z$ has a supremum,

then $f$ has a fixed point above $z$. Furthermore, there exists a maximal fixed point of $f$.

In complete lattices the above theorem takes the following form.

Theorem 6.4 (Birkhoff-Tarski). Let $(X, \leq)$ be a complete lattice and $f: X \rightarrow X$ an increasing mapping. Then there exist a smallest fixed point $\underline{x}$ and a greatest fixed point $\bar{x}$ for $f$, given by $\underline{x}=\inf \left\{f^{n}(\top): n \in \mathbb{N}\right\}$ and by $\bar{x}=\sup \left\{f^{n}(\perp): n \in \mathbb{N}\right\}$, where $\perp$ denotes the least element of $X$ and $T$ the greatest one.

Furthermore, the set of fixed points of the mapping $f$ is a complete lattice. 
Proof. Since $\underline{x} \leq \top$ it follows $f(\underline{x}) \leq f(\top)$. Also $\underline{x} \leq f^{n}(\top)$ implies $f(\underline{x}) \leq f^{n+1}(\top)$ for all $n \in \mathbb{N}$. Consequently, $f(\underline{x}) \leq \underline{x}$. By the definition of $\underline{x}, \underline{x} \leq f(\top)$, so that $f(\underline{x})=\underline{x}$.

The case of $\bar{x}$ can be treated similarly.

In the following theorem one asks a kind of Scott continuity for the mapping $f$.

Theorem 6.5 (Tarski-Kantorovich). Let $(X, \leq)$ be a poset such that every countable chain in $X$ has a supremum and $f: X \rightarrow X$ a mapping that preserves the suprema of countable chains. If there exists $z \in X$ such that $z \leq f(z)$, then $f$ has a fixed point. Moreover, $z_{0}:=\sup \left\{f^{n}(z): n \in \mathbb{N}\right\}$ is the least fixed point of $f$ in $\uparrow z$.

Proof. We include the simple proof of this result following [77. Since $f$ preserves suprema of countable chains it follows that it is increasing. From $z \leq f(z)$ follows $f(z) \leq f^{2}(z)$ and, by induction, $f^{n-1}(z) \leq f^{n}(z)$ for all $n \in \mathbb{N}$, showing that $\left\{f^{n}(z): n \in \mathbb{N}\right\}$ is a chain in $\uparrow z$. If $x_{0}:=\sup \left\{f^{n}(z): n \in \mathbb{N}\right\}$, then, by hypothesis, $f\left(x_{0}\right)=\sup \left\{f^{n+1}(z): n \in \mathbb{N}\right\}=x_{0}$.

Let $x_{1} \geq z$ be a fixed point of $f$. Then $f(z) \leq f\left(x_{1}\right)=x_{1}$ and, by induction $f^{n}(z) \leq x_{1}$ for all $n \in \mathbb{N}$, that is $x_{1}$ is an upper bound for $\left\{f^{n}(z): n \in \mathbb{N}\right\}$ and so $x_{0} \leq x_{1}$.

Remark 6.6. In Theorem 6.5 it is sufficient to suppose that every countable chain in $\uparrow z$ has a supremum and that $f$ preserves these suprema.

6.2. Converse results. Apparently, the first converse result in this area was obtained by Davis [54].

Theorem 6.7. A lattice $(X, \leq)$ is complete if and only if every increasing mapping $f$ : $X \rightarrow X$ has a fixed point.

By a result of Frink [69] (see Theorem 5.17), a lattice $(X, \leq)$ is complete if and only if it is compact with respect to the interval topology. Consequently Theorem 6.7 admits the following reformulation.

Theorem 6.8. A lattice $(X, \leq)$ is compact in its interval topology if and only if every increasing mapping $f: X \rightarrow X$ has a fixed point.

Extensions to lower semi-lattices of this result as well as of Birkhoff-Tarski fixed point theorem, Theorem 6.4, were given by Ward [215]. Recall that a lower semi-lattice (semilattice in short) is a poset $(X, \leq)$ such that $x \wedge y$ exists for every $x, y \in X$. It is called complete if every nonempty subset of $X$ has an infimum.

Theorem 6.9 ([215]).

(1) A semi-lattice $(X, \leq)$ is complete if and only if for every $x \in X, \downarrow x$ is compact with respect to the interval topology.

(2) A semi-lattice $(X, \leq)$ is compact with respect to the interval topology if and only if every increasing mapping $f: X \rightarrow X$ has a fixed point.

Smithson [186] extended Davis' results to the case of set-valued mappings. Wolk [218] obtained also characterizations of directed completeness of posets (called by him Dedekind completeness) in terms of fixed points of monotonic maps acting on them.

We mention also the following result of Jachymski [95], connecting several properties equivalent to FPP. A periodic point for a mapping $f: X \rightarrow X$ is an element $x_{0} \in X$ such that $f^{k}\left(x_{0}\right)=x_{0}$, for some $k \in \mathbb{N}$. The set of periodic points is denoted by $\operatorname{Per}(f)$ while the set of fixed points is denoted by $\operatorname{Fix}(f)$. It is obvious that a fixed point is a periodic point with $k=1$. 
Theorem 6.10. Let $X$ be a nonempty abstract set and $f$ be a self-map of $X$. The following statements are equivalent.

(1) $\operatorname{Per}(f)=\operatorname{Fix}(f) \neq \emptyset$.

(2) (Zermelo) There exists a partial ordering $\preceq$ such that every chain in $(X, \preceq)$ has a supremum and $f$ is progressive with respect to $\preceq($ i.e. $x \preceq f(x), x \in X)$.

(3) (Caristi) There exists a complete metric $d$ and a lower semicontinuous function $\varphi: X \rightarrow \mathbb{R}$ such that $f$ satisfies condition (2.11).

(4) There exists a complete metric $d$ and a d-Lipschitz function $\varphi: X \rightarrow \mathbb{R}$ such that $f$ satisfies condition (2.11) and $f$ is nonexpansive with respect to d; i.e.;

$$
d(f(x), f(y)) \leq d(x, y) \text { for all } x, y \in X
$$

(5) (Hicks-Rhoades) For each $\alpha \in(0,1)$ there exists a complete metric $d$ such that $f$ is nonexpansive with respect to $d$ and

$$
d\left(f(x), f^{2}(x)\right) \leq \alpha d(x, f(x)) \text { for all } x \in X
$$

(6) There exists a complete metric $d$ such that $f$ is continuous with respect to $d$ and for each $x \in X$ the sequence $\left(f^{n}(x)\right)_{n=1}^{\infty}$ is convergent (the limit may depend on $x)$.

For two nonempty sets $A, B$ denote by $B^{A}$ the family of all mappings from $A$ to $B$, $B^{A}:=\{f: f: A \rightarrow B\}$.

Let $\left(X,\left(\rho_{i}\right)_{i \in I}\right)$ be a uniform space where $\left\{\rho_{i}: i \in I\right\}$ is a family of semi-metrics generating the uniformity of $X$. Define a partial order $\preceq$ on $X \times \mathbb{R}_{+}^{I}$ by

$$
(x, \varphi) \leq(y, \psi) \Longleftrightarrow \forall i \in I, \quad \rho_{i}(x, y) \leq \varphi(i)-\psi(i),
$$

for $x, y \in X$ and $\varphi, \psi \in \mathbb{R}_{+}^{I}$.

If $(X, \rho)$ is a metric space (i.e. $I$ is a singleton and $\rho_{1}=\rho$ is a metric), then the relation order (6.1) becomes

$$
(x, \alpha) \leq(y, \beta) \Longleftrightarrow \rho(x, y) \leq \alpha-\beta,
$$

for $x, y \in X$ and $\alpha, \beta \in \mathbb{R}_{+}$, an order considered by Ekeland in connection with his variational principle.

An ordered set $(X, \leq)$ is called Dedekind $\sigma$-complete if every bounded increasing sequence has a supremum and every bounded decreasing sequence has an infimum. Also one says that a sequence $\left(x_{n}\right)$ in $X$ is order convergent (o-convergent) to $x \in X$ if there exists the sequences $\left(y_{n}\right)$ and $\left(z_{n}\right)$ in $X$ such that

(i) $y_{n} \leq y_{n+1} \leq x_{n} \leq z_{n+1} \leq z_{n}$ for all $n \in \mathbb{N}$ and

(ii) $\sup _{n} y_{n}=x=\inf _{n} z_{n}$,

(i.e., the sequential version of (5.5)).

DeMarr [56] proved the following results concerning the order (6.2).

Theorem 6.11. Let $(X, \rho)$ be a metric space and $\preceq$ the order on $X \times \mathbb{R}_{+}$defined by (6.2) . Then the following hold.

(1) A sequence $\left(x_{n}\right)$ in $X$ is convergent to $x \in X$ if and only if the sequence $\left(\left(x_{n}, 0\right)\right)_{n \in \mathbb{N}}$ is o-convergent to $(x, 0)$ in $(X \times \mathbb{R}, \preceq)$. 
(2) The metric space $(X, \rho)$ is complete if and only if the ordered set $(X \times \mathbb{R}, \preceq)$ is Dedekind $\sigma$-complete.

He used these results to prove the following fixed point theorem.

Theorem $6.12([56])$. Let $(X, \rho)$ be a complete metric space and $\preceq$ the order on $X \times \mathbb{R}_{+}$ defined by (6.2). If $f: X \times \mathbb{R} \rightarrow X \times \mathbb{R}$ is an increasing map for which there exist $\xi_{0}, \xi_{1} \in X \times \mathbb{R}$ such that $\xi_{0} \leq f\left(\xi_{0}\right) \leq f\left(\xi_{1}\right) \leq \xi_{1}$, then $f$ has a fixed point.

Jachymski [92] extended these results to uniform spaces and the order (6.1).

Theorem 6.13. Let $\left(X,\left(\rho_{i}\right)_{i \in I}\right)$ be a uniform space and $\preceq$ the order on $X \times \mathbb{R}_{+}^{I}$ defined by (6.1). Then the following are equivalent.

(1) Every sequence $\left(x_{n}\right)$ in $X$ such that $\sum_{n=1}^{\infty} \rho_{i}\left(x_{n}, x_{n+1}\right)<\infty$, for all $i \in I$, is convergent.

(2) Every countable chain in $\left(X \times \mathbb{R}_{+}^{I}, \preceq\right)$ has a supremum.

(3) Every increasing sequence in $\left(X \times \mathbb{R}_{+}^{I}, \preceq\right)$ has a supremum.

In particular, if the space $X$ is sequentially complete, then each of the above conditions holds.

In the case of a metric space $(X, \rho)$ one obtains a characterization of completeness.

Theorem 6.14. Let $(X, \rho)$ be a metric space and $\preceq$ the order on $X \times \mathbb{R}_{+}$defined by (6.2). Then the following are equivalent.

(1) The metric space $X$ is complete.

(2) Every chain in $\left(X \times \mathbb{R}_{+}, \preceq\right)$ has a supremum.

(3) Every countable chain in $\left(X \times \mathbb{R}_{+}, \preceq\right)$ has a supremum.

(4) Every increasing sequence in $\left(X \times \mathbb{R}_{+}, \preceq\right)$ has a supremum.

Jachymski applied these results to obtain proofs of fixed point results for mappings on partially ordered sets. In their turn, these order fixed point results were applied to obtain simpler proofs and extensions to various fixed point results in metric and in uniform spaces, see, for instance, the papers by Jachymski [90, 91, 92, 94], and the references cited therein.

Klimeš [119] has found a common extension to Theorems 6.1 and 6.3. Let $(X, \leq)$ be partially ordered. A mapping $f: X \rightarrow X$ is called partially isotone if for all $x, y \in X$

$$
(x \leq y \wedge x \leq f(y) \wedge f(x) \leq y) \Rightarrow f(x) \leq f(y) .
$$

It is obvious that increasing mappings, "progressive" mappings (satisfying $x \leq f(x)$ ) and "regressive" mappings (satisfying $f(x) \leq x$ ) are partially isotone.

The mapping $f$ is called comparable if $x$ is comparable with $f(x)$ for every $x \in X$. The partially ordered set $X$ is called inductive if every chain in $X$ has an upper bound, and semiuniform if for every chain $C$ in $X$ the set of upper bounds of $C$ is downward directed.

Klimeš [119] proved that:

- every relatively isotone self-mapping on a complete lattice has a fixed point (Theorem 1.2);

- if the partially ordered set $X$ is chain complete (i.e. every chain in $X$, including the empty chain, has a supremum) then every relatively isotone self-mapping on $X$ has a fixed point (Theorem 1.6);

- a lattice $X$ is complete if and only if every comparable self-mapping on $X$ has a fixed point (Theorem 2.2);

- a semiuniformly partially ordered set $X$ is chain complete if and only if every relatively isotone self-mapping on $X$ has a fixed point (Theorem 2.3). 
In [120] he considered ascending maps $f: X \rightarrow X$, meaning that $f(x) \leq y$ implies $f(x) \leq f(y)$ for all $x, y \in X$, and proved that the partially ordered set $X$ is inductive if and only if every ascending self-mapping on $X$ has a fixed point. For other related results, see [117] and [118]. For instance, in [118] one considers mappings $f: X \rightarrow X, X$ a partially ordered set, such that $x \leq y$ and $x \leq f(x)$ implies $f(x) \leq f(y)$, called by the author extensively isotone.

6.3. Fixed points in ordered metric spaces. The title of this subsection could be a little confusing - in contrast to ordered Banach spaces, or Banach lattices, it concerns a metric space $(X, \rho)$ equipped with an order relation $\leq$ that does not have any connection with the metric structure. Fixed points are proved for mappings $f: X \rightarrow X$ which are monotonic (increasing or decreasing) with respect to the order and contractive with respect to the metric, but in a restricted manner in the following sense: there exists $0 \leq \alpha<1$ such that

$$
\rho(f(x), f(y)) \leq \alpha \rho(x, y) \text { if } x, y \in X \text { are comparable (i.e. } x \leq y \text { or } y \leq x) .
$$

Theorem 6.15. Let $(X, \rho)$ be a complete metric space equipped with a partial order $\leq$ and $f: X \rightarrow X$ a mapping satisfying (6.5). Then the following results hold.

(1) ([150]) If the mapping $f$ is increasing and continuous and there exists $x_{0} \in X$ such that $x_{0} \leq f\left(x_{0}\right)$, then $f$ has a fixed point.

(2) ([150]) Suppose that for every increasing sequence $\left(x_{n}\right)$ in $X$ converging to some $x \in X$ it holds $x_{n} \leq x$ for all $n \in \mathbb{N}$. If $f$ is increasing and there exists $x_{0} \in X$ such that $x_{0} \leq f\left(x_{0}\right)$, then $f$ has a fixed point.

(3) ([169]) Suppose that every pair $x, y$ of elements in $X$ has an upper bound or a lower bound. If $f$ is continuous and monotone (i.e. either increasing or decreasing) and there exists $x_{0} \in X$ such that $x_{0} \leq f\left(x_{0}\right)$ or $f\left(x_{0}\right) \leq x_{0}$, then $f$ has a unique fixed point $\bar{x}$ and for every $x \in X$ the sequence $\left(f^{n}(x)\right)_{n \in \mathbb{N}}$ converges to $\bar{x}$.

(4) ([111]) Assume that the ordered set $(X, \leq)$ admits a smallest element $x_{0}$. Then the conclusions from (3) hold for every continuous increasing function $f: X \rightarrow X$ satisfying (6.5).

Proof. (1) The proof is simple. Since $f$ is increasing

$$
x_{0} \leq f\left(x_{0}\right) \Rightarrow f\left(x_{0}\right) \leq f^{2}\left(x_{0}\right) \Rightarrow f^{2}\left(x_{0}\right) \leq f^{3}\left(x_{0}\right) \Rightarrow \ldots
$$

showing that the sequence $\left(f^{n}\left(x_{0}\right)\right)$ is increasing. By (6.5)

$$
\rho\left(f^{n}\left(x_{0}\right), f^{n+1}\left(x_{0}\right)\right) \leq \alpha \rho\left(f^{n-1}\left(x_{0}\right), f^{n}\left(x_{0}\right)\right) \leq \cdots \leq \alpha^{n} \rho\left(x_{0}, f\left(x_{0}\right)\right),
$$

for all $n \in \mathbb{N}$. But then, by the triangle inequality,

$$
\rho\left(f^{n}\left(x_{0}\right), f^{n+k}\left(x_{0}\right)\right) \leq\left(\alpha^{n}+\alpha^{n+1}+\cdots+\alpha^{n+k-1}\right) d\left(x_{0}, f\left(x_{0}\right)\right) \longrightarrow 0
$$

as $n \rightarrow \infty$, uniformly with respect to $k \in \mathbb{N}$, which shows that $\left(f^{n}\left(x_{0}\right)\right)$ is a Cauchy sequence and so, by the completeness of the metric space $X$, it converges to some $\bar{x} \in X$. By the continuity of $f$,

$$
f(\bar{x})=f\left(\lim _{n} f^{n}\left(x_{0}\right)\right)=\lim _{n} f^{n+1}\left(x_{0}\right)=\bar{x} .
$$

(2) As in the proof of $(1)$, the sequence $\left(f^{n}\left(x_{0}\right)\right)$ is increasing and convergent to some $\bar{x} \in X$. By hypothesis, it follows $f^{n}\left(x_{0}\right) \leq \bar{x}$ for all $n \in \mathbb{N}$, so that,

$$
\rho\left(f^{n+1}\left(x_{0}\right), f(\bar{x})\right) \leq \alpha \rho\left(f^{n}\left(x_{0}\right), \bar{x}\right) \rightarrow 0 \text { as } n \rightarrow \infty .
$$

It follows $\rho(\bar{x}, f(\bar{x}))=0$, that is $f(\bar{x})=\bar{x}$. 
(3) Suppose that $f$ is increasing and that there exists $x_{0} \in X$ such that $x_{0} \leq f\left(x_{0}\right)$. Then $\left(f^{n}\left(x_{0}\right)\right)$ is an increasing sequence, convergent to some $\bar{x} \in X$ which is a fixed point for $f$. The proof will be done if we show that, for every $x \in X$, the sequence $\left(f^{n}(x)\right)$ is convergent to $\bar{x}$.

Let $x \in X$. If $x \leq x_{0}$, then $f^{n}(x) \leq f^{n}\left(x_{0}\right)$ so that, by (6.5),

$$
\rho\left(f^{n}(x), f^{n}\left(x_{0}\right)\right) \leq \alpha \rho\left(f^{n-1}(x), f^{n-1}\left(x_{0}\right)\right) \leq \cdots \leq \alpha^{n} \rho\left(x, x_{0}\right) \rightarrow 0 .
$$

It follows $\lim _{n} f^{n}(x)=\lim _{n} f^{n}\left(x_{0}\right)=\bar{x}$. The situation is the same if $x \geq x_{0}$.

If $x \in X$ is not comparable to $x_{0}$, then, by hypothesis, $x$ and $x_{0}$ have a lower bound or an upper bound in $(X, \leq)$.

If they have a lower bound $x_{1}$, then $x_{1} \leq x_{0}$ and $x_{1} \leq x$, so that by the first part of the proof

$$
\bar{x}=\lim _{n} f^{n}\left(x_{0}\right)=\lim _{n} f^{n}\left(x_{1}\right)=\lim _{n} f^{n}(x) .
$$

The situation is the same if $x$ and $x_{0}$ have an upper bound $x_{2}$ in $X$.

(4) In this case $x_{0} \leq f\left(x_{0}\right)$ and, for every $x \in X, x_{0} \leq x$, so we can proceed as in the proof of $(3)$.

Remark 6.16. Usually results as those from Theorem 6.15 are called fixed point of RanReurings type [169].

Refinements of the above results were given in [96], [149], [151] and [166].

\section{Partial Metric SPACES}

These spaces were introduced by Matthews [139, 140, 141, 142] in connection with his research on computer science. They are only $T_{0}$ topological spaces, a feature that fits the needs of denotational semantics of dataflow networks. In this section we shall first present the basic notions and results following [42, [140, 141] and [142] (see also the books [112] and [179]). Although all the included results on partial metric spaces can be found in the papers of Matthews or in other ones dealing with fixed point results in such spaces, we include full proofs of the results, for reader's convenience. At the same time, different approaches concerning convergence of sequences and completeness notions in partial metric spaces, used by various authors, are put in a proper light.

7.1. Definition and topological properties. Let $X$ be a nonempty set.

Definition 7.1. A mapping $p: X \times X \rightarrow \mathbb{R}_{+}$satisfying the following conditions

(PM1) $x=y \Longleftrightarrow p(x, x)=p(y, y)=p(x, y) \quad$ (indistancy implies equality);

(PM2) $0 \leq p(x, x) \leq p(x, y)$ (nonnegativity and small self-distances);

(PM3) $p(x, y)=p(y, x)$ (symmetry);

(PM4) $p(x, z) \leq p(x, y)+p(y, z)-p(y, y)$ (triangularity),

for all $x, y, z \in Z$, is called a partial metric on $X$. The pair $(X, p)$ is called a partial metric space.

This means that, in contrast to the metric case, one admits the possibility that $d(x, x)>$ 0 for some points $x \in X$, a property called "self-distancy".

A point $x \in X$ is called

- complete if $p(x, x)=0$, and

- partial if $p(x, x)>0$, giving an explanation for the term "partial" coined by Matthews. 
The following property follows from (PM2) and (PM1).

$$
p(x, y)=0 \Longrightarrow x=y \text { (indistancy implies equality). }
$$

The following characterization of partial metric spaces is given by M. \& V. Anisiu [20].

Theorem 7.2. A function $p: X \times X \rightarrow[0, \infty)$ is a partial metric on $X$ if and only if there exist a metric $d$ and a nonexpansive with respect to $d$ function $\varphi: X \rightarrow[0, \infty)$, such that

$$
p(x, y)=d(x, y)+\varphi(x)+\varphi(y) \quad \text { for all } \quad x, y \in X .
$$

Furthermore, $d$ and $\varphi$ are uniquely determined by $p$.

The following two examples of partial metric spaces are related to some questions in theoretical computer science.

Example 7.3. The function $p: 2^{\mathbb{N}} \times 2^{\mathbb{N}} \rightarrow[0, \infty)$ defined by

$$
p(x, y)=1-\sum_{n \in x \cap y} 2^{-n},
$$

with the convention that the sum over the empty set is 0 , is a partial metric on $2^{\mathbb{N}}$.

Example 7.4. For a nonempty set $S$ let $S^{\infty}=S^{*} \cup S^{\mathbb{N}}$ be the set of all finite (belonging to $S^{*}$ ) or infinite sequences (belonging to $S^{\mathbb{N}}$ ). The length $\ell(x)$ of a finite sequence $x=$ $\left(x_{1}, x_{2}, \ldots, x_{n}\right)$ is $n$ and the length of an infinite sequence $x: \mathbb{N} \rightarrow S$ is $\infty$. Put $i(x, y)=$ $\sup \left\{n \in \mathbb{N}: n \leq \ell(x) \wedge \ell(y), x_{j}=y_{j}, \forall j<n\right\}$, and define

$$
p(x, y)=2^{-i(x, y)}, \quad x, y \in S^{\infty},
$$

with the convention $2^{-\infty}=0$, is a partial metric on $S^{\infty}$.

The function $p$ is a metric on $S^{\mathbb{N}}$, called the Baire metric, and a partial metric on $S^{*} \cup S^{\mathbb{N}}$, because $p(x, x)=2^{-n}>0$ for $x=\left(x_{1}, \ldots, x_{n}\right) \in S^{*}$.

We define the open balls as in the metric case:

$$
B_{p}(x, \varepsilon):=\{y \in X: p(x, y)<\varepsilon\},
$$

for $x \in S^{\infty}$ and $\varepsilon>0$.

Remark 7.5. In this case the possibility that $B_{p}(x, \varepsilon)=\emptyset$ is not excluded.

If $p(x, x)>0$, then $B_{p}(x, \varepsilon)=\emptyset$ for every $0<\varepsilon \leq p(x, x)$.

If $B_{p}(x, \varepsilon) \neq \emptyset$, then $x \in B_{p}(x, \varepsilon)$.

Indeed, by (PM2), $p(x, y) \geq p(x, x) \geq \varepsilon$ for every $y \in X$ implies $B_{p}(x, \varepsilon)=\emptyset$. Also, if $y \in B_{p}(x, \varepsilon)$, then, again by (PM2), $p(x, x) \leq p(x, y)<\varepsilon$, i.e. $x \in B_{p}(x, \varepsilon)$.

Consider also the balls

$$
B_{p}^{\prime}(x, \varepsilon):=\{y \in X: p(x, y)<\varepsilon+p(x, x)\},
$$

for $x \in X$ and $\varepsilon>0$. It is obvious that $x \in B_{p}^{\prime}(x, \varepsilon)$.

The following proposition contains some properties of these two kinds of balls.

Proposition 7.6. Let $(X, p)$ be a partial metric space.

(1) If $y \in B_{p}(x, \varepsilon)$ then

$$
y \in B_{p}(y, \delta) \subseteq B_{p}(x, \varepsilon),
$$

where $\delta:=\varepsilon-p(x, y)+p(y, y)>0$. 
(2) The balls $B_{p}$ and $B_{p}^{\prime}$ are related by the following equalities:

$$
B_{p}^{\prime}(x, \varepsilon)=B_{p}(x, \varepsilon+p(x, x)),
$$

and

$$
B_{p}(x, \varepsilon)= \begin{cases}B_{p}^{\prime}(x, \varepsilon-p(x, x)) & \text { if } \varepsilon>p(x, x), \\ \emptyset & \text { if } 0<\varepsilon \leq p(x, x) .\end{cases}
$$

(3) For the balls $B^{\prime}, z \in B_{p}^{\prime}(z, r)$ for any $z \in X$ and $r>0$. Also if $\varepsilon^{\prime}>0$ and $y \in B_{p}^{\prime}\left(x, \varepsilon^{\prime}\right)$, then

$$
B_{p}^{\prime}\left(y, \delta^{\prime}\right) \subseteq B_{p}^{\prime}\left(x, \varepsilon^{\prime}\right),
$$

where $\delta^{\prime}:=\varepsilon^{\prime}+p(x, x)-p(x, y)$.

Proof. (1) Let $\delta:=\varepsilon-p(x, y)+p(y, y)$. Then $\delta>0$ (because $p(x, y)<\varepsilon$ ) and $p(y, y)<\delta$, so that $y \in B_{p}(y, \delta)$.

If $z \in B_{p}(y, \delta)$, then the inequalities

$$
p(y, z)<\varepsilon-p(x, y)+p(y, y) \text { and } p(x, z) \leq p(x, y)+p(y, z)-p(y, y),
$$

yield by addition, $p(x, z)<\varepsilon$, that is $z \in B_{p}(x, \varepsilon)$ and so $B_{p}(y, \delta) \subseteq B_{p}(x, \varepsilon)$.

The equalities from (2) are obvious by the definitions of the corresponding balls (see also Remark (7.5).

The inclusion from (3) follows by (1) and (2), but it can be also proved directly. We have

$$
y \in B_{p}^{\prime}\left(x, \varepsilon^{\prime}\right) \Longleftrightarrow p(x, y)<\varepsilon^{\prime}+p(x, x)
$$

and

so that

$$
z \in B_{p}^{\prime}\left(y, \delta^{\prime}\right) \Longleftrightarrow p(y, z)<\delta^{\prime}+p(y, y)
$$

$$
\begin{aligned}
p(x, z) & \leq p(x, y)+p(y, z)-p(y, y) \\
& <p(x, y)+\varepsilon^{\prime}+p(x, x)-p(x, y)+p(y, y)-p(y, y) \\
& =\varepsilon^{\prime}+p(x, x),
\end{aligned}
$$

which shows that $z \in B_{p}^{\prime}\left(x, \varepsilon^{\prime}\right)$.

Now we introduce the topology of a partial-metric space and present some of its properties.

Theorem 7.7. Let $(X, p)$ be a partial metric space.

(1) The family of open balls

$$
\mathcal{B}:=\left\{B_{p}(x, \varepsilon), x \in X, \varepsilon>0\right\}
$$

is the basis of a topology on $X$, denoted by $\tau_{p}$ (sometimes by $\tau(p)$ ).

(2) The family $\mathcal{B}^{\prime}$ of the sets

$$
\mathcal{B}^{\prime}:=\left\{B_{p}^{\prime}(x, \varepsilon), x \in X, \varepsilon>0\right\}
$$

is also a basis for the topology $\tau_{p}$.

(3) The balls $B_{p}(x, \varepsilon), B_{p}^{\prime}(x, \varepsilon)$ are open and for every $x \in X$ and $\varepsilon>0$. The family $\mathcal{V}_{p}(x)$ of neighborhoods of $x$ is given by

$$
\begin{aligned}
\mathcal{V}_{p}(x) & =\left\{V \subseteq X: \exists \delta>0, x \in B_{p}(x, \delta) \subseteq V\right\} \\
& =\left\{V \subseteq X: \exists \varepsilon>0, B_{p}^{\prime}(x, \varepsilon) \subseteq V\right\} .
\end{aligned}
$$


(4) The topology $\tau_{p}$ is $T_{0}$ and satisfies the first axiom of countability, i.e., every point in $X$ has a countable basis of neighborhoods.

Proof. (1) By [109, Th. 11, p. 47] a family $\mathcal{B} \subseteq 2^{X}$ is a basis for a topology $\tau$ on a set $X$ if and only if the following two conditions are satisfied:

(B1) $\quad X=\bigcup \mathcal{B}$

(B2) for every $B_{1}, B_{2} \in \mathcal{B}$ and $x \in B_{1} \cap B_{2}$ there exists $B \in \mathcal{B}$ such that

$$
x \in B \subseteq B_{1} \cap B_{2} .
$$

Since $x \in B_{p}(x, 1+p(x, x))$, it follows $X=\bigcup\left\{B_{p}(x, 1+p(x, x)): x \in X\right\} \subseteq \bigcup \mathcal{B}$, so (B1) holds.

Also, by Proposition [7.6.(1), for any $z \in B_{p}\left(x, \varepsilon_{1}\right) \cap B_{p}\left(y, \varepsilon_{2}\right)$,

$$
z \in B_{p}(z, \delta) \subseteq B_{p}\left(x, \varepsilon_{1}\right) \cap B_{p}\left(y, \varepsilon_{2}\right),
$$

where

$$
\delta:=p(z, z)+\min \left\{\varepsilon_{1}-p(x, z), \varepsilon_{2}-p(y, z)\right\},
$$

so (B2) is satisfied too.

These two properties show that the family (7.5) forms a basis of a topology $\tau_{p}$ on $X$, that is, every set in $\tau_{p}$ can be written as a union of open balls of the form $B_{p}(x, \varepsilon)$.

(2) Let us show that $\mathcal{B}^{\prime}$ is also a basis. Since $x \in B_{p}^{\prime}(x, \varepsilon)$ it follows $X=\bigcup\left\{B_{p}^{\prime}(x, 1)\right.$ : $x \in X\} \subseteq \cup \mathcal{B}^{\prime}$.

Also, by Proposition 7.6.(3),

$$
z \in B_{p}^{\prime}\left(z, \delta^{\prime}\right) \subseteq B_{p}^{\prime}\left(x_{1}, \varepsilon_{1}^{\prime}\right) \cap B_{p}^{\prime}\left(x_{2}, \varepsilon_{2}^{\prime}\right),
$$

where $\delta^{\prime}=\min \left\{\delta_{1}^{\prime}, \delta_{2}^{\prime}\right\}$ for $\delta_{i}^{\prime}:=\varepsilon_{i}^{\prime}+p\left(x_{i}, x_{i}\right)-p\left(x_{i}, z\right), i=1,2$.

The equivalence of two bases $\mathcal{B}, \mathcal{B}^{\prime}$ means that

$$
\forall B \in \mathcal{B} \text { and } \forall x \in B, \exists B^{\prime} \in \mathcal{B}^{\prime} \text { such that } x \in B^{\prime} \subseteq B
$$

and, conversely,

$$
\forall B^{\prime} \in \mathcal{B}^{\prime} \text { and } \forall x \in B^{\prime}, \exists B \in \mathcal{B} \text { such that } x \in B \subseteq B^{\prime} .
$$

If $y \in B_{p}(x, \varepsilon)$, then it is easy to check that

$$
y \in B_{p}^{\prime}(y, \gamma) \subseteq B_{p}(x, \varepsilon)
$$

where $\gamma:=\varepsilon-p(x, y)$.

Conversely, if $y \in B_{p}^{\prime}(x, \varepsilon)$, then $B_{p}^{\prime}(x, \varepsilon)=B_{p}(y, \varepsilon+p(y, y))$, so that

$$
y \in B_{p}(y, \varepsilon+p(y, y))=B_{p}^{\prime}(x, \varepsilon) \text {. }
$$

(3) By Proposition [7.6.(1) for every $y \in B_{p}(x, \varepsilon)$ we have $y \in B_{p}\left(y, \delta_{y}\right) \subseteq B_{p}(x, \varepsilon)$, where where $\delta_{y}=\varepsilon-p(x, y)+p(y, y)$, showing that $B_{p}(x, \varepsilon)$ is open. The openness of $B_{p}^{\prime}(x, \varepsilon)$ can be obtained in the same way from Proposition 7.6. (3).

Since the open balls form a basis of the topology $\tau_{p}, V \in \mathcal{V}_{p}(x)$ if and only if there exists $y \in X$ and $\varepsilon>0$ such that $x \in B_{p}(y, \varepsilon) \subseteq V$. Appealing again to Proposition 7.6, it follows that $x \in B_{p}(x, \delta) \subseteq B_{p}(y, \varepsilon) \subseteq V$, where $\delta=\varepsilon-p(x, y)+p(x, x)$.

(4) We have to show that for any pair $x, y$ of distinct points in $X$ there exists a $\tau_{p}$-open set containing exactly one of them.

Let $x \neq y$ be two points in $X$. Then by (PM1) and (PM2) either $p(x, x)<p(x, y)$ or $p(y, y)<p(x, y)$.

Suppose $p(x, x)<p(x, y)$ and let $\varepsilon:=(p(x, x)+p(x, y)) / 2$. Then

$$
2 p(x, x)<p(x, x)+p(x, y)=2 \varepsilon \Rightarrow p(x, x)<\varepsilon \Longleftrightarrow x \in B_{p}(x, \varepsilon) .
$$


On the other side

$$
p(x, y)>p(x, x)=2 \varepsilon-p(x, y) \Rightarrow p(x, y)>\varepsilon \Rightarrow y \notin B_{p}(x, \varepsilon) .
$$

The case $p(y, y)<p(x, y)$ can be treated similarly.

The family $\left\{B_{p}^{\prime}(x, 1 / n): n \in \mathbb{N}\right\}$ is a countable basis of neighborhoods at $x$.

Remark 7.8. We adopt the convention that $\bigcup\left\{A_{i}: i \in \emptyset\right\}=\emptyset$ (implying, by de Morgan rules, $\left.\bigcap\left\{A_{i}: i \in \emptyset\right\}=X\right)$, and so $\emptyset$ belongs to the family of arbitrary unions of sets in $\mathcal{B}$. If one considers only unions over nonempty index sets, then we must say that the family $\mathcal{B}$ plus the empty set generates the topology $\tau_{p}$.

7.2. Convergent sequences, completeness and the Contraction Principle. The convergence of sequences with respect to $\tau_{p}$ can be characterized in the following way.

Proposition 7.9. Let $(X, p)$ be a partial metric space. A sequence $\left(x_{n}\right)$ in $X$ is $\tau_{p^{-}}$ convergent to $x \in X$ if and only if

$$
\lim _{n \rightarrow \infty} p\left(x, x_{n}\right)=p(x, x) .
$$

Proof. Suppose that $x_{n} \stackrel{\tau_{p}}{\longrightarrow} x$. Given $\varepsilon>0$ let $n_{0} \in \mathbb{N}$ be such that , for all $n \geq n_{0}, x_{n} \in$ $B_{p}(x, \varepsilon+p(x, x)) \Longleftrightarrow p\left(x, x_{n}\right)<\varepsilon+p(x, x)$. Taking into account (PM2), it follows

$$
0 \leq p\left(x, x_{n}\right)-p(x, x)<\varepsilon,
$$

for all $n \geq n_{0}$, showing that (7.8) holds.

Conversely, suppose that (7.8) holds and let $V \in \mathcal{V}_{p}(x)$. Since, by Theorem [7.7. $(2), \mathcal{B}^{\prime}$ is also a basis for the topology $\tau_{p}$, there exists $\varepsilon>0$ such that $B_{p}^{\prime}(x, \varepsilon) \subseteq V$. Let $n_{0} \in \mathbb{N}$ be such that $0 \leq p\left(x, x_{n}\right)-p(x, x)<\varepsilon$ for all $n \geq n_{0}$. Then

$$
0 \leq p\left(x, x_{n}\right)-p(x, x)<\varepsilon \Longleftrightarrow p\left(x, x_{n}\right)<\varepsilon+p(x, x) \Longleftrightarrow x_{n} \in B_{p}^{\prime}(x, \varepsilon) \subseteq V,
$$

for all $n \geq n_{0}$, proving that $x_{n} \stackrel{\tau_{p}}{\longrightarrow} x$.

Remark 7.10. Since the topology $\tau_{p}$ of a partial metric space is only $T_{0}$, a convergent sequence can have many limits. In fact, if $x_{n} \stackrel{\tau_{p}}{\rightarrow} x$, then $x_{n} \stackrel{\tau_{p}}{\rightarrow} y$ for any $y \in X$ such that $p(x, y)=p(y, y)$.

Indeed

$0 \leq p\left(y, x_{n}\right)-p(y, y) \leq p(y, x)+p\left(x, x_{n}\right)-p(x, x)-p(y, y)=p\left(x, x_{n}\right)-p(x, x) \longrightarrow 0$.

For a nonempty subset $Y$ of a partial metric space $(X, p)$ and $x \in X$ put

$$
p(x, Y)=\inf \{p(x, y): y \in Y\} .
$$

The closure of a subset of $X$ admit the following characterization in terms of this distance function.

Proposition 7.11 ([11], Lemma 2). Let $Y$ be a nonempty subset of a partial metric space $(X, p)$ and $x \in X$. Then

$$
x \in \bar{Y} \Longleftrightarrow p(x, Y)=p(x, x) .
$$

Proof. Because

$$
p(x, x) \leq p(x, y) \text { for all } y \in X \text {, }
$$


we have the following equivalences:

$$
\begin{aligned}
p(x, x)=p(x, Y) & \Longleftrightarrow \forall \varepsilon>0, \exists y \in Y, p(x, y)<\varepsilon+p(x, x) \\
& \Longleftrightarrow \forall \varepsilon>0, Y \cap B_{p}^{\prime}(x, \varepsilon) \neq \emptyset \\
& \Longleftrightarrow x \in \bar{Y} .
\end{aligned}
$$

To obtain uniqueness and to define a reasonable notion of completeness, a stronger notion of convergence is needed.

Definition 7.12. One says that a sequence $\left(x_{n}\right)$ in a partial metric space converges properly to $x \in X$ if and only if

$$
\lim _{n \rightarrow \infty} p\left(x, x_{n}\right)=p(x, x)=\lim _{n \rightarrow \infty} p\left(x_{n}, x_{n}\right) .
$$

In other words, $\left(x_{n}\right)$ converges properly to $x$ if and only if $\left(x_{n}\right)$ converges to $x$ with respect to $\tau_{p}$ and further

$$
\lim _{n \rightarrow \infty} p\left(x_{n}, x_{n}\right)=p(x, x) .
$$

Proposition 7.13. Let $(X, p)$ be a partial metric space and $\left(x_{n}\right)$ a sequence in $X$ that converges properly to $x \in X$. Then

(i) the limit is unique, and

(ii) $\lim _{m, n \rightarrow \infty} p\left(x_{m}, x_{n}\right)=p(x, x)$.

Proof. Suppose that $x, y \in X$ are such that $\left(x_{n}\right)$ converges properly to both $x$ and $y$. Then

$$
p(x, y) \leq p\left(x, x_{n}\right)+p\left(x_{n}, y\right)-p\left(x_{n}, x_{n}\right) \longrightarrow p(y, y) \text { as } n \rightarrow \infty,
$$

implies $p(x, y) \leq p(y, y)$. By (PM2) this implies $p(x, y)=p(y, y)$.

Similarly,

$$
p(x, y) \leq p\left(x, y_{n}\right)+p\left(y_{n}, y\right)-p\left(y_{n}, y_{n}\right) \longrightarrow p(x, x) \text { as } n \rightarrow \infty,
$$

implies $p(x, y) \leq p(x, x)$, and so $p(x, y)=p(x, x)$.

It follows

$$
p(x, y)=p(x, x)=p(y, y),
$$

so that, by (PM1), $x=y$.

To prove (ii) observe that

$$
p\left(x_{m}, x_{n}\right) \leq p\left(x_{m}, x\right)+p\left(x, x_{n}\right)-p(x, x)
$$

implying

$$
p\left(x_{m}, x_{n}\right)-p(x, x) \leq p\left(x_{m}, x\right)+p\left(x, x_{n}\right)-2 p(x, x) \longrightarrow 0 \text { as } m, n \rightarrow \infty .
$$

Also, by (PM2) and (PM4),

$$
p(x, x) \leq p\left(x, x_{n}\right) \leq p\left(x, x_{m}\right)+p\left(x_{m}, x_{n}\right)-p\left(x_{m}, x_{m}\right)
$$

so that

$$
p(x, x)-p\left(x_{m}, x_{n}\right) \leq p\left(x, x_{m}\right)-p\left(x_{m}, x_{m}\right) \longrightarrow 0 \text { as } m, n \rightarrow \infty .
$$

Consequently, $\lim _{m, n \rightarrow \infty} p\left(x_{m}, x_{n}\right)=p(x, x)$. 
Remark 7.14. Some authors take the condition (ii) from Proposition 7.13 in the definition of a properly convergent sequence. As it was shown this is equivalent to the condition from Definition 7.12

The definition of Cauchy sequences in partial metric spaces takes the following form.

Definition 7.15. A sequence $\left(x_{n}\right)$ in a partial metric space $(X, p)$ is called a Cauchy sequence if there exists $a \geq 0$ in $\mathbb{R}$ such that for every $\varepsilon>0$ there exists $n_{\varepsilon} \in \mathbb{N}$ with

$$
\left|p\left(x_{n}, x_{m}\right)-a\right|<\varepsilon
$$

for all $m, n \geq n_{\varepsilon}$, written also as $\lim _{m, n \rightarrow \infty} p\left(x_{n}, x_{m}\right)=a$.

The partial metric space $(X, p)$ is called complete if every Cauchy sequence is properly convergent to some $x \in X$.

A mapping $f$ on a partial metric space $(X, p)$ is called a contraction if there exists $0 \leq \alpha<1$ such that

$$
p(f(x), f(y)) \leq \alpha p(x, y)
$$

for all $x, y \in X$.

The analogue of Banach Contraction Principle holds in partial metric spaces too.

Theorem 7.16 ([140, [142]). Let $(X, p)$ be a complete partial metric spaces. Then every contraction $f: X \rightarrow X$ has a fixed point $x_{0}$ such that $p\left(x_{0}, x_{0}\right)=0$.

Proof. (Sketch) Let $f$ be an $\alpha$-contraction on $X$ with $0 \leq \alpha<1$.

One shows first that for every $z \in X$ the sequence of iterates $\left(f^{n}(z)\right)$ satisfies the condition

$$
\lim _{m, n \rightarrow \infty} p\left(f^{n}(z), f^{m}(z)\right)=0
$$

i.e. it is Cauchy. By the completeness of $(X, p)$ there exists $x_{0} \in X$ such that

$$
0=\lim _{n \rightarrow \infty} p\left(f^{n}(z), f^{n}(z)\right)=p\left(x_{0}, x_{0}\right)=\lim _{n \rightarrow \infty} p\left(x_{0}, f^{n}(z)\right) .
$$

But

$$
\begin{aligned}
0 & \leq p\left(x_{0}, f\left(x_{0}\right)\right) \leq p\left(x_{0}, f^{n}\left(x_{0}\right)\right)+p\left(f^{n}\left(x_{0}\right), f\left(x_{0}\right)\right)-p\left(f^{n}\left(x_{0}\right), f^{n}\left(x_{0}\right)\right) \\
& \leq p\left(x_{0}, f^{n}\left(x_{0}\right)\right)+\alpha p\left(f^{n-1}\left(x_{0}\right), x_{0}\right)-p\left(f^{n}\left(x_{0}\right), f^{n}\left(x_{0}\right)\right) \longrightarrow 0 \quad \text { as } n \rightarrow \infty .
\end{aligned}
$$

It follows $p\left(x_{0}, f\left(x_{0}\right)\right)=0=p\left(x_{0}, x_{0}\right)$. The relations $0 \leq p\left(f\left(x_{0}\right), f\left(x_{0}\right)\right) \leq \alpha p\left(x_{0}, x_{0}\right)=$ 0 imply $p\left(f\left(x_{0}\right), f\left(x_{0}\right)\right)=0$, so that, by (PM1), $f\left(x_{0}\right)=x_{0}$.

Remark 7.17. O'Neill [155] considered partial metrics that take values in $\mathbb{R}$ (not in $\mathbb{R}_{+}$ as in the case of Matthews' partial metric) and related them to domain theory. These kind of spaces are called by some authors dualistic partial metric space. The extension of Banach fixed point theorem to this setting was given by Oltra and Valero 153 (see also [212]). In this case the contraction condition is given by

$$
\exists 0 \leq \alpha<1 \text { such that } \forall x, y \in X, \quad|p(f(x), f(y))| \leq \alpha|p(x, y)| .
$$

Extensions of various fixed point results from metric spaces to partial metric spaces were given by $\mathrm{O}$. Valero in cooperation with other mathematicians, see [6, 7, 182, 183, 213] (see also [178]). 
7.3. Topology and order on partial metric spaces. In this subsection we shall examine the behavior of the specialization order (5.2) with respect to the topology $\tau(p)$ generated by a partial metric $p$.

Proposition 7.18. Let $(X, p)$ be a partial metric space and $\leq_{p}$ the specialization order on $X$.

(1) The specialization order can be characterized by the following condition

$$
x \leq_{p} y \Longleftrightarrow p(x, x)=p(x, y) .
$$

(2) The open balls $B_{p}(x, \varepsilon)$ and $B_{p}^{\prime}(x, \varepsilon)$ are upward closed. Consequently every $\tau_{p}$-open sets is upward closed.

(3) The Alexandrov topology $\tau_{a}\left(\leq_{p}\right)$ generated by $\leq_{p}$ (see Proposition 5.9) is finer than $\tau(p)$. The equality $\tau(p)=\tau_{a}\left(\leq_{p}\right)$ holds if and only if

$$
\forall x \in X, \exists \varepsilon_{x}>0, \quad B_{p}\left(x, \varepsilon_{x}\right)=\uparrow x .
$$

Proof. (1) Suppose $x \leq_{p} y$. By definition $x \leq_{p} y \Longleftrightarrow x \in \overline{\{y\}}$ and

$$
\begin{aligned}
x \in \overline{\{y\}} & \Longleftrightarrow \forall \varepsilon>0,\{y\} \cap B_{p}^{\prime}(x, \varepsilon) \neq \emptyset \\
& \Longleftrightarrow \forall \varepsilon>0, p(x, y)<\varepsilon+p(x, x) \\
& \Longleftrightarrow p(x, y) \leq p(x, x) \\
& \Longleftrightarrow p(x, y)=p(x, x) \quad \text { (by (PM2)) } .
\end{aligned}
$$

(2) Let $y \in B_{p}(x, \varepsilon)$ and $y \leq_{p} z \Longleftrightarrow p(y, z)=p(y, y)$. Then

$$
p(x, z) \leq p(x, y)+p(y, z)-p(y, y)=p(x, y)<\varepsilon,
$$

that is $z \in B_{p}(x, \varepsilon)$. The proof is similar for $B_{p}^{\prime}(x, \varepsilon)$.

If $U \subseteq X$ is $\tau_{p}$-open and $x \in U$, then there exists $\varepsilon_{x}>0$ such that $x \in B_{p}\left(x, \varepsilon_{x}\right) \subseteq U$. If $x \leq_{p} y$, then, since $B_{p}\left(x, \varepsilon_{x}\right)$ is upward closed, $y \in B_{p}\left(x, \varepsilon_{x}\right) \subseteq U$. Consequently $U$ is upward closed.

(3) Since the Alexandov topology is the finest such that the induced order specialization agrees with $\leq_{p}$ (Proposition [5.9), it follows $\tau(p) \subseteq \tau_{a}\left(\leq_{p}\right)$.

Suppose now that the condition (7.16) holds and let $Z \in \tau_{a}\left(\leq_{p}\right)$. Since the $\tau_{a}\left(\leq_{p}\right)$-open sets are upward closed (see Remark [5.10), it follows

$$
Z=\bigcup\{\uparrow x: x \in Z\}=\bigcup\left\{B_{p}\left(x, \varepsilon_{x}\right): x \in Z\right\} \in \tau(p) .
$$

Consequently, $\tau_{a}\left(\leq_{p}\right) \subseteq \tau(p)$, so that $\tau_{a}\left(\leq_{p}\right)=\tau(p)$.

Conversely, suppose that $\tau_{a}\left(\leq_{p}\right)=\tau(p)$. Then for every $x \in X, \uparrow x \in \tau(p)$, implying the existence of $\varepsilon_{x}>0$ such that $x \in B_{p}\left(x, \varepsilon_{x}\right) \subseteq \uparrow x$.

If $y \in \uparrow x$, then $p(x, y)=p(x, x)<\varepsilon_{x}$, that is $y \in B_{p}\left(x, \varepsilon_{x}\right)$, showing that $\uparrow x \subseteq B_{p}\left(x, \varepsilon_{x}\right)$ and so $B_{p}\left(x, \varepsilon_{x}\right)=\uparrow x$.

Remark 7.19. In terms of the specialization order $\leq_{p}$ of a partial metric space $(X, p)$, Remark 7.10 says in fact that if a sequence $\left(x_{n}\right)$ in $X$ converges to $x \in X$, then it converges to every $y$ with $y \leq_{p} x$. Also, the equalities (7.13) say that if $\left(x_{n}\right)$ converges properly to $x$ and $y$, then $x \leq_{p} y$ and $y \leq_{p} x$, and so $x=y$.

Proposition 5.4 allows us to characterize the $T_{1}$ property of partial metric spaces.

Proposition $7.20(79])$. Let $(X, p)$ be a partial metric space. 
(1) The space $X$ is $T_{1}$ if and only if

$$
p(x, x)<p(x, y)
$$

for every $x \neq y$ in $X$.

(2) The following are equivalent:

(i) the space $X$ is $T_{2}$;

(ii) for every $x \neq y$ in $X$

$$
\inf \{p(x, z)-p(x, x)+p(y, z)-p(y, y): z \in X\}>0
$$

(iii) every convergent sequence has a unique limit.

Proof. (1) By Proposition 5.4 and the definition (7.15) of the specialization order $\leq_{p}$ in $(X, p)$, the topology $\tau_{p}$ is $T_{1}$ if and only if $p(x, y)=p(x, x)$ implies $x=y$, for all $x, y \in X$. This is equivalent to $p(x, x)<p(x, y)$ for all $x \neq y$ in $X$.

(2) (i) $\Rightarrow$ (ii). Suppose that $\tau_{p}$ is $T_{2}$ and let $x \neq y$ be two points in $X$. Then there exists $\varepsilon>0$ such that

$$
B_{p}^{\prime}(x, \varepsilon) \cap B_{p}^{\prime}(y, \varepsilon)=\emptyset .
$$

This means that, for every $z \in X$,

$$
p(x, z) \geq \varepsilon+p(x, x) \text { or } p(y, z) \geq \varepsilon+p(y, y),
$$

implying

$$
\inf \{p(x, z)-p(x, x)+p(y, z)-p(y, y): z \in X\} \geq \varepsilon>0 .
$$

(ii) $\Rightarrow$ (iii). We prove the equivalent implication $\neg$ (iii) $\Rightarrow \neg$ (ii). Suppose that there exist a sequence $\left(x_{n}\right)$ in $X$ and $x \neq y$ in $X$ such that

$$
\begin{aligned}
& x_{n} \rightarrow x \Longleftrightarrow 0 \leq p\left(x, x_{n}\right)-p(x, x) \rightarrow 0 \text { and } \\
& x_{n} \rightarrow y \Longleftrightarrow 0 \leq p\left(y, x_{n}\right)-p(y, y) \rightarrow 0 .
\end{aligned}
$$

It follows

$$
\begin{aligned}
0 & \leq \inf \{p(x, z)-p(x, x)+p(y, z)-p(y, y): z \in X\} \\
& \leq \inf \left\{p\left(x, x_{n}\right)-p(x, x)+p\left(y, x_{n}\right)-p(y, y): n \in \mathbb{N}\right\}=0,
\end{aligned}
$$

that is, (7.18) fails.

(iii) $\Rightarrow$ (i). We prove again the equivalent implication $\neg$ (i) $\Rightarrow \neg($ iii).

If $X$ is not $T_{2}$, then there exist $x \neq y$ in $X$ such that $B_{p}^{\prime}(x, 1 / n) \cap B_{p}^{\prime}(y, 1 / n) \neq \emptyset$ for every $n \in \mathbb{N}$. Taking a point $x_{n}$ in each of these sets, it follows that, for every $n \in \mathbb{N}$,

$$
\begin{aligned}
& 0 \leq p\left(x, x_{n}-p(x, x)<\frac{1}{n}\right. \text { and } \\
& 0 \leq p\left(y, x_{n}-p(y, y)<\frac{1}{n},\right.
\end{aligned}
$$

which shows that $x_{n} \rightarrow x$ and $x_{n} \rightarrow y$.

Remark 7.21. The paper [79] also contains a discussion on the validity of $T_{3}$ and $T_{4}$ separation axioms as well as on the second countability and separability of partial metric space. 
7.4. The specialization order in quasi-metric spaces. In this subsection we shall describe the specialization order in a quasi-metric space.

Proposition 7.22. Let $(X, q)$ be a quasi-metric space.

(1) The specialization order $\leq_{q}$ corresponding to $q$ is given by

$$
x \leq_{q} y \Longleftrightarrow q(x, y)=0 \text {. }
$$

(2) Every open set is upward closed.

Proof. (1) For $x, y \in X$,

$$
\begin{aligned}
x \leq_{q} y & \Longleftrightarrow x \in \overline{\{y\}} \Longleftrightarrow \forall \varepsilon>0, y \in B_{q}(x, \varepsilon) \\
& \Longleftrightarrow \forall \varepsilon>0, q(x, y)<\varepsilon \Longleftrightarrow q(x, y)=0 .
\end{aligned}
$$

(2) Let us show first that an open ball $B_{q}(x, \varepsilon)$ is upward closed. Indeed, $y \in B_{q}(x, \varepsilon)$ and $y \leq_{q} z$ imply

$$
q(x, z) \leq q(x, y)+q(y, z)=q(x, y)<\varepsilon .
$$

Now if $U \subseteq X$ is $\tau_{q}$-open, then for every $x \in U$ there exists $\varepsilon_{x}>0$ such that $B_{q}\left(x, \varepsilon_{x}\right) \subseteq$ $U$. If $x \leq_{q} y$, then $y \in B_{q}\left(x, \varepsilon_{x}\right) \subseteq U$.

Remark 7.23. If $q$ is only a quasi-semimetric (see Definition 4.1), then (7.19) defines only a preorder $\leq_{q}$, which is an order if and only if $q$ is a quasi-metric.

Indeed

$$
\begin{aligned}
\left(x \leq_{q} y \wedge y \leq_{q} x\right) & \Longleftrightarrow(q(x, y)=0 \wedge q(y, x)=0) \\
& \Longleftrightarrow x=y .
\end{aligned}
$$

A contraction principle holds in this case too. A mapping $f$ on a quasi-metric space $(X, q)$ is called a contraction if there exists $\alpha \in[0,1)$ such that

$$
q(f(x), f(y)) \leq \alpha q(x, y),
$$

for all $x, y \in X$.

Theorem 7.24 (Contraction Principle in quasi-metric spaces, [141]). Let $(X, q)$ be a quasi-metric space such that the associated metric space $\left(X, q^{s}\right)$ is complete. Then every contraction on $(X, q)$ has a fixed point.

7.5. Partial metrics and quasi-metrics. In this subsection we put in evidence some relations between partial metrics and quasi-metrics.

Proposition 7.25. Let $(X, p)$ be a partial metric space. Then the mapping $q: X^{2} \rightarrow \mathbb{R}_{+}$ given by

$$
q(x, y)=p(x, y)-p(x, x), \quad x, y \in X,
$$

is a quasi-metric on $X$. The topology $\tau(p)$ generated by $p$ agrees with the topology $\tau(q)$ generated by $q$ and the corresponding specialization orders $\leq_{p}$ and $\leq_{q}$ coincide as well.

Proof. It is a routine verification to show that the mapping $q$ defined by (7.21) is a quasimetric on $X$.

For $0<\varepsilon \leq p(x, x), B_{p}(x, \varepsilon)=\emptyset \in \tau(q)$. If $\varepsilon>p(x, x)$, then $B_{p}(x, \varepsilon)=B_{q}(x, \varepsilon-$ $p(x, x)) \in \tau(q)$, relations that imply $\tau(p) \subseteq \tau(q)$.

Since, for every $\varepsilon>0, B_{q}(x, \varepsilon)=B_{p}(x, \varepsilon+p(x, x)) \in \tau(p)$, it follows $\tau(q) \subseteq \tau(p)$. 
Taking into account (7.15)

$$
\begin{aligned}
x \leq_{p} y & \Longleftrightarrow p(x, y)=p(x, x) \Longleftrightarrow q(x, y)=0 \Longleftrightarrow \forall \varepsilon>0, y \in B_{q}(x, \varepsilon) \\
& \Longleftrightarrow x \in \overline{\{y\}} \bar{q}^{q} \Longleftrightarrow x \leq_{q} y .
\end{aligned}
$$

Remark 7.26. It follows that

$$
q^{s}(x, y)=q(x, y)+q(y, x)=2 p(x, y)-p(x, x)-p(y, y), x, y \in X,
$$

is a metric on $X$, called the associate metric to the partial metric $p$.

Indeed, writing $p^{s}$ as

$$
q^{s}(x, y)=p(x, y)-p(x, x)+p(x, y)-p(y, y), x, y \in X,
$$

and taking into account (PM2), it follows $q^{s}(x, y) \geq 0$. Also, $q^{s}(x, y)=0$ implies $p(x, x)=$ $p(x, y)=p(y, y)$, so that, by (PM1), $x=y$. The symmetry of $q^{s}$ is obvious and the triangle inequality $q^{s}(x, z) \leq q^{s}(x, y)+q^{s}(y, z)$ follows from (PM4).

The next result shows that the completeness of the partial metric space $(X, p)$ is equivalent to the completeness of the associate metric space $\left(X, q^{s}\right)$.

Proposition 7.27. Let $(X, p)$ be a partial metric space and $q^{s}$ the associated metric to $p$ given by (7.22).

(1) ([154]) The following inequality

$$
|p(x, y)-p(z, w)| \leq q^{s}(x, z)+q^{s}(y, w),
$$

is true for all $x, y, z, w \in X$.

(2) The convergence and completeness properties of the spaces $(X, p)$ and $\left(X, q^{s}\right)$ are related in the following way:

(i) a sequence $\left(x_{n}\right)$ in $X$ is properly convergent to $x \in X$ if and only if $x_{n} \stackrel{q^{s}}{\rightarrow} x$;

(ii) a sequence $\left(x_{n}\right)$ in $X$ is p-Cauchy if and only if it is $q^{s}$-Cauchy;

(iii) the partial metric space $(X, p)$ is complete if and only if the associated metric space $\left(X, q^{s}\right)$ is complete.

(3) The mapping $p(\cdot, \cdot)$ is continuous on $X \times X$ with respect to the metric $d((x, y),(z, w))=$ $q^{s}(x, z)+q^{s}(y, w)$ generating the product topology $\tau\left(q^{s}\right) \times \tau\left(q^{s}\right)$ on $X \times X$.

In particular, the mapping $\beta: X \rightarrow[0, \infty)$, given by $\beta(x)=p(x, x), x \in X$, is $q^{s}$-continuous.

Proof. (1) By the triangle inequality (PM4)

$$
\begin{aligned}
p(x, y) & \leq p(x, z)+p(z, y)-p(z, z) \\
& \leq p(x, z)+p(z, w)+p(w, y)-p(w, w)-p(z, z),
\end{aligned}
$$

so that

$$
\begin{aligned}
p(x, y)-p(z, w) & \leq p(x, z)+\underbrace{p(x, z)-p(x, x)}_{\geq 0}-p(z, z)+ \\
& +p(y, w)+\underbrace{p(y, w)-p(y, y)}_{\geq 0}-p(w, w) \\
& =q^{s}(x, z)+q^{s}(y, w) .
\end{aligned}
$$


Because

$$
p(z, w)-p(x, y) \leq q^{s}(z, x)+q^{s}(w, y)=q^{s}(x, z)+q^{s}(y, w),
$$

the inequality (7.24) follows.

(2).(i) By definition

$$
x_{n} \stackrel{q^{s}}{\rightarrow} x \Longleftrightarrow p\left(x_{n}, x\right)-p(x, x)+p\left(x_{n}, x\right)-p\left(x_{n}, x_{n}\right) \longrightarrow 0 .
$$

Since $p\left(x_{n}, x\right)-p(x, x) \geq 0$ and $p\left(x_{n}, x\right)-p\left(x_{n}, x_{n}\right) \geq 0$ the last condition from above is equivalent to

$$
\left\{\begin{array} { l } 
{ p ( x _ { n } , x ) \longrightarrow p ( x , x ) } \\
{ p ( x _ { n } , x ) - p ( x _ { n } , x _ { n } ) \longrightarrow 0 }
\end{array} \Longleftrightarrow \left\{\begin{array}{l}
p\left(x_{n}, x\right) \longrightarrow p(x, x) \\
p\left(x_{n}, x_{n}\right) \longrightarrow p(x, x)
\end{array}\right.\right.
$$

that is, to the fact that $\left(x_{n}\right)$ converges properly to $x$.

(ii) I. Any p-Cauchy sequence is $q^{s}$-Cauchy.

Let $\left(x_{n}\right)$ be a $p$-Cauchy sequence in $X$, that is

$$
\lim _{m, n \rightarrow \infty} p\left(x_{m}, x_{n}\right)=a,
$$

for some $a \in \mathbb{R}_{+}$. Then $\lim _{k \rightarrow \infty} p\left(x_{k}, x_{k}\right)=a$, so that

$$
q^{s}\left(x_{m}, x_{n}\right)=2 p\left(x_{m}, x_{n}\right)-p\left(x_{n}, x_{n}\right)-p\left(x_{m}, x_{m}\right) \longrightarrow 0 \text { as } m, n \rightarrow \infty,
$$

which shows that the sequence $\left(x_{n}\right)$ is $q^{s}$-Cauchy.

II. Any $q^{s}$-Cauchy sequence is p-Cauchy.

Let $\left(x_{n}\right)$ be a $q^{s}$-Cauchy sequence in $X$. We show now that the net $\left(p\left(x_{m}, x_{n}\right)\right)_{(m, n) \in \mathbb{N}^{2}}$ is Cauchy in $\mathbb{R}_{+}$. By (7.24),

$$
\left|p\left(x_{m}, x_{n}\right)-p\left(x_{m^{\prime}}, x_{n^{\prime}}\right)\right| \leq q^{s}\left(x_{m}, x_{m^{\prime}}\right)+q^{s}\left(x_{n}, x_{n^{\prime}}\right) .
$$

Given $\varepsilon>0$ let $n_{0} \in \mathbb{N}$ be such that

$$
q^{s}\left(x_{m}, x_{m^{\prime}}\right)<\varepsilon / 2 \text { and } q^{s}\left(x_{n}, x_{n^{\prime}}\right)<\varepsilon / 2,
$$

for all $m, m^{\prime} \geq n_{0}$ and all $n, n^{\prime} \geq n_{0}$. But then, the inequality (7.25) yields

$$
\left|p\left(x_{m}, x_{n}\right)-p\left(x_{m^{\prime}}, x_{n^{\prime}}\right)\right|<\varepsilon,
$$

for all $m, m^{\prime}, n, n^{\prime} \geq n_{0}$.

Since the net $\left(p\left(x_{m}, x_{n}\right)\right)_{(m, n) \in \mathbb{N}^{2}}$ is Cauchy in $\mathbb{R}_{+}$, it converges to some $a \in \mathbb{R}_{+}$, which means that the sequence $\left(x_{n}\right)$ is $p$-Cauchy.

(iii) This follows from the definition of the completeness of the partial metric space $(X, p)$ and from (i) and (ii).

(3) If $q^{s}\left(x_{n}, x\right) \rightarrow 0$ and $q^{s}\left(y_{n}, y\right) \rightarrow 0$, then, by (7.24),

$$
\left|p\left(x_{n}, y_{n}\right)-p(x, y)\right| \leq q^{s}\left(x_{n}, x\right)+q^{s}\left(y_{n}, y\right) \longrightarrow 0,
$$

proving the $q^{s}$-continuity of $p(\cdot, \cdot)$ at $(x, y) \in X \times X$.

Remark 7.28. Definition 7.15 of a Cauchy sequence in a partial metric space is taken from [142] (see also [42]). In [140] the following equivalent definition is proposed: a sequence $\left(x_{n}\right)$ in a partial metric space $(X, p)$ is called a Cauchy sequence if for every $\varepsilon>0$ there exists $n_{\varepsilon} \in \mathbb{N}$ such that

$$
0 \leq p\left(x_{n}, x_{m}\right)-p\left(x_{m}, x_{m}\right)<\varepsilon,
$$

for all $m, n \geq n_{\varepsilon}$. 
Indeed, the equality (7.23) shows that this is equivalent to the fact that $\left(x_{n}\right)$ is $q^{s}$ Cauchy, which in its turn is equivalent to the fact that $\left(x_{n}\right)$ is $p$-Cauchy.

Remark 7.29. Another metric on a partial metric space $(X, p)$ is given by $d(x, y)=0$ if $x=y$ and $d(x, y)=p(x, y)$ for $x \neq y$. In this case $\tau_{q^{s}} \subseteq \tau_{d}$ and the metric space $(X, d)$ is complete if and only if the partial metric space $(X, p)$ is complete. This result can be used to show that some fixed points results in partial metric spaces can be obtained directly from their analogues in the metric case, see [78. A similar situation occurs in the case of the so called cone-metric spaces, see, for instance, the survey paper [98].

Example 7.30. Some topological properties of the partial metric spaces $2^{\mathbb{N}}$ and $S^{\infty}$ from Examples 7.3 and 7.4 are examined in [154].

In the case of the partial metric $\left(2^{\mathbb{N}}, p\right)$, the associated quasi-metric is

$$
q(x, y)=\sum_{k \in x \backslash y} 2^{-k}
$$

and the associated metric is

$$
q^{s}(x, y)=\sum_{k \in x \Delta y} 2^{-k}
$$

where $x \Delta y=(x \backslash y) \cup(y \backslash x)$ is the symmetric difference of the sets $x, y \subseteq \mathbb{N}$.

It is shown in [154] that:

- The spaces $2^{\mathbb{N}}$ and $S^{\infty}$ are complete.

- The associated metric space $\left(2^{\mathbb{N}}, q^{S}\right)$ is compact (and so separable).

- The associated metric space $\left(S^{\infty}, q^{s}\right)$ is separable if and only if the set $S$ is countable.

- The associated metric space $\left(S^{\infty}, q^{s}\right)$ is compact if and only if the set $S$ is finite.

7.6. The existence of suprema in partial metric spaces. In this subsection we shall prove that every increasing sequence in a partial metric space has a supremum and it is properly convergent to its supremum. We agree to call a mapping $f:\left(X_{1}, p_{1}\right) \rightarrow\left(X_{2}, p_{2}\right)$ properly continuous if $\left(f\left(x_{n}\right)\right)$ properly converges to $f(x)$ for every sequence $\left(x_{n}\right)$ in $X_{1}$ properly convergent to $x$.

Proposition 7.31. Let $(X, p)$ be a partial metric space and $\leq_{p}$ the specialization order corresponding to $p$.

(1) If $(X, p)$ is complete, then every increasing sequence $x_{1} \leq_{p} x_{2} \leq_{p} \ldots$ in $X$ has a supremum $x$ and the sequence $\left(x_{n}\right)$ converges properly to $x$.

(2) Let $\left(X_{1}, p_{1}\right),\left(X_{2}, p_{2}\right)$ be complete partial metric spaces with the specialization orders $\leq_{1}, \leq_{2}$, respectively, and $f:\left(X_{1}, p_{1}\right) \rightarrow\left(X_{2}, p_{2}\right)$ a mapping. If $f$ is properly continuous and monotonic, then $f$ preserves suprema of increasing sequences, that is, $\sup _{n} f\left(x_{n}\right)=f(x)$ for every increasing sequence $x_{1} \leq_{1} x_{2} \leq_{1} \ldots$ in $X_{1}$ with $\sup _{n} x_{n}=x$

Proof. (1) We show first that the sequence $\left(x_{n}\right)$ is Cauchy. Indeed,

$$
x_{n} \leq_{p} x_{n+k} \Longleftrightarrow p\left(x_{n}, x_{n+k}\right)-p\left(x_{n}, x_{n}\right)=0,
$$

so that, taking into account Remark 7.28, it follows that $\left(x_{n}\right)$ is Cauchy. The completeness hypothesis implies the existence of $x \in X$ such that the sequence $\left(x_{n}\right)$ is properly convergent to $x$, that is

$$
\lim _{n} p\left(x, x_{n}\right)=p(x, x)=\lim _{n} p\left(x_{n}, x_{n}\right) .
$$


We show that $x=\sup _{n} x_{n}$, that is

(i) $x_{n} \leq x$ for all $n \in \mathbb{N}$;

(ii) if $x_{n} \leq y$ for all $n \in \mathbb{N}$, then $x \leq y$.

We have for all $n, k \in \mathbb{N}$

$$
\begin{aligned}
p\left(x_{n}, x\right) & \leq p\left(x_{n}, x_{n+k}\right)+p\left(x_{n+k}, x\right)-p\left(x_{n+k}, x_{n+k}\right) \\
& =p\left(x_{n}, x_{n}\right)+p\left(x_{n+k}, x\right)-p\left(x_{n+k}, x_{n+k}\right) .
\end{aligned}
$$

Letting $k \rightarrow \infty$ and taking into account (7.26), one obtains $p\left(x_{n}, x\right) \leq p\left(x_{n}, x_{n}\right)$, so that, by (PM2) from Definition 7.1, $p\left(x_{n}, x\right)=p\left(x_{n}, x_{n}\right)$, that is $x_{n} \leq_{p} x$.

Suppose now that $x_{n} \leq_{p} y$ for all $n \in \mathbb{N}$. Then

$$
\begin{aligned}
p(x, y) & \leq p\left(x, x_{n}\right)+p\left(x_{n}, y\right)-p\left(x_{n}, x_{n}\right) \\
& =p\left(x_{n}, y\right)=p\left(x_{n}, x_{n}\right),
\end{aligned}
$$

for all $n \in \mathbb{N}$. Letting $n \rightarrow \infty$ one obtains (by (7.26), $p(x, y) \leq p(x, x)$. It follows $p(x, y)=p(x, x)$, that is $x \leq_{p} y$. Consequently, both conditions (i) and (ii) from (7.27) hold.

(2) Let $x_{1} \leq_{1} x_{2} \leq_{1} \ldots$ be an increasing sequence in $X$ with $\sup _{n} x_{n}=x$. Then $\left(x_{n}\right)$ is $p_{1}$-properly convergent to $x$. Then the sequence $\left(f\left(x_{n}\right)\right)$ is $\leq_{2}$-increasing and properly convergent to $f(x)$. By (1), this implies that $\sup _{n} f\left(x_{n}\right)=f(x)$.

Remark 7.32. It is possible that the property from the first statement of Proposition 7.31 characterizes the completeness of the partial metric space $(X, p)$ (like in Theorem 6.14). Concerning the second statement, I don't know whether the Scott continuity is equivalent to the continuity of the mapping $f$.

7.7. Caristi's fixed point theorem and completeness in partial metric spaces. In this subsection we shall present, following Romaguera [170] the equivalence of Caristi's fixed point theorem to the completeness of the underlying partial metric space.

Let $(X, p)$ be a partial metric space. Recall the Caristi condition for a mapping $f$ : $X \rightarrow X:$

$\left(\operatorname{Car}_{\varphi}\right)$

$$
p(x, f(x)) \leq \varphi(x)-\varphi(f(x)),
$$

for all $x \in X$. Here $\varphi$ is a function $\varphi: X \rightarrow \mathbb{R}$. According to the continuity properties of the function $\varphi$ we distinct two kinds of Caristi conditions. One says that the mapping $f$ is

- $p$-Caristi if (Car ${ }_{\varphi}$ holds for some $p$-lsc bounded from below function $\varphi: X \rightarrow \mathbb{R}$;

- $q^{s}$-Caristi if $\left(\operatorname{Car}_{\varphi}\right)$ holds for some $q^{s}$-lsc bounded from below function $\varphi: X \rightarrow \mathbb{R}$,

where $q^{s}$ is the metric associated to $p$ by (7.22).

As it was shown in [170] the completeness of a partial metric space $(X, p)$ cannot be characterized by the existence of fixed points of $p$-Caristi mappings.

Example 7.33. Consider the set $\mathbb{N}$ with the partial metric $p(m, n)=\max \left\{m^{-1}, n^{-1}\right\}$. The associated metric $q^{s}$ is given by $q^{s}(m, n)=\left|m^{-1}-n^{-1}\right|, m, n \in \mathbb{N}$. If $0<\varepsilon<$ $[n(n+1)]^{-1}$, then $B_{q^{s}}(n, \varepsilon)=\{n\}$, that is the topology $\tau\left(q^{s}\right)$ is the discrete metric on $\mathbb{N}$, and so the only convergent sequences are the ultimately constant ones. The space $\left(\mathbb{N}, q^{s}\right)$ is not complete because the sequence $x_{n}=n, n \in \mathbb{N}$, is $q^{s}$-Cauchy and not $q^{s}$-convergent. On the other side there are no $p$-Caristi maps on $\mathbb{N}$. 
To obtain a characterization of this kind, another notion is needed.

Definition 7.34. Let $(X, p)$ be a partial metric space. A sequence $\left(x_{n}\right)$ in $X$ is called 0 - Cauchy if and only if $\lim _{m, n \rightarrow \infty} p\left(x_{m}, x_{n}\right)=0$. The partial metric space $(X, p)$ is called 0 -complete if every 0-Cauchy sequence $\left(x_{n}\right)$ is convergent with respect to $\tau_{p}$ to some $x \in X$ such that $p(x, x)=0$.

Remark 7.35. The above definition is given in [170]. Taking into account Proposition 7.27, the following assertions hold:

$$
\begin{aligned}
& \left\{\begin{array}{l}
\lim _{m, n \rightarrow \infty} p\left(x_{n}, x_{m}\right)=0, \\
\lim _{n \rightarrow \infty} p\left(x, x_{n}\right)=p(x, x), \\
p(x, x)=0,
\end{array}\right. \\
& \Rightarrow\left\{\begin{array}{l}
\left(x_{n}\right) \text { is } q^{s} \text {-Cauchy, and } \\
x_{n} \stackrel{q^{s}}{\rightarrow} x .
\end{array}\right.
\end{aligned}
$$

Consequently, a partial metric space $(X, p)$ is 0-complete if and only if every 0-Cauchy sequence is properly convergent if and only if every 0-Cauchy sequence is $q^{s}$-convergent.

Remark 7.36. It is obvious that a complete partial metric space is 0 -complete, but the converse is not true (see [170]).

Notice also the following property.

Remark 7.37 ([1]). Let $(X, p)$ be a partial metric space, $\left(x_{n}\right)$ a sequence in $X$ and $x \in X$. If $\lim _{n \rightarrow \infty} p\left(x_{n}, x\right)=0$, then $\lim _{n \rightarrow \infty} p\left(x_{n}, y\right)=p(x, y)$ for every $y \in Y$.

Indeed,

$$
p\left(x_{n}, y\right) \leq p\left(x_{n}, x\right)+p(x, y)-p(x, x) \leq p\left(x_{n}, x\right)+p(x, y),
$$

implies $p\left(x_{n}, y\right)-p(x, y) \leq p\left(x_{n}, x\right)$, while

$$
p(x, y) \leq p\left(x, x_{n}\right)+p\left(x_{n}, y\right)-p\left(x_{n}, x_{n}\right) \leq p\left(x, x_{n}\right)+p\left(x_{n}, y\right),
$$

implies $p(x, y)-p\left(x_{n}, y\right) \leq p\left(x, x_{n}\right)$.

Consequently

$$
\left|p(x, y)-p\left(x_{n}, y\right)\right| \leq p\left(x, x_{n}\right) \longrightarrow 0 .
$$

The characterization result is the following one.

Theorem 7.38 ([170, [112]). Let $(X, p)$ be a partial metric space. Then $(X, p)$ is 0 complete if and only if every $q^{s}$-Caristi mapping on $X$ has a fixed point.

Proof. Suppose that $(X, p)$ is 0-complete and let $f: X \rightarrow X$ be a $q^{s}$-Caristi mapping for some $q^{s}$-lsc bounded for below function $\varphi: X \rightarrow \mathbb{R}$. For $x \in X$ let

$$
A_{x}:=\{y \in X: p(x, y)+\varphi(y) \leq \varphi(x)\} .
$$

Then, by $\overline{\left.\operatorname{Car}_{\varphi}\right)}, f(x) \in A_{x}$ and $A_{x}$ is $q^{s}$-closed because, taking into account Proposition 7.27, the mapping $p(x, \cdot)+\varphi(\cdot)$ is $q^{s}$-lsc.

Starting with an arbitrary $x_{0} \in X$ we shall construct inductively a sequence of $q^{s}$-closed sets $A_{x_{n}}$ such that, for all $k \in \mathbb{N}$,

(i) $x_{k} \in A_{x_{k-1}}$ and $A_{x_{k}} \subseteq A_{x_{k-1}}$

(ii) $p\left(x_{k}, x\right)<\frac{1}{2^{k}}$ for all $x \in A_{x_{k}}$. 
Suppose that $x_{k}$ and $A_{x_{k}}, k=0,1, \ldots, n$, satisfy the conditions (7.28). Choose $x_{n+1} \in$ $A_{x_{n}}$ such that

$$
\varphi\left(x_{n+1}\right)<\inf \varphi\left(A_{x_{n}}\right)+\frac{1}{2^{n+1}}
$$

If $y \in A_{x_{n+1}}$, then

$$
\begin{aligned}
p\left(x_{n}, y\right) & \leq p\left(x_{n}, x_{n+1}\right)+p\left(x_{n+1}, y\right)-p\left(x_{n+1}, x_{n+1}\right) \\
& \leq \varphi\left(x_{n}\right)-\varphi\left(x_{n+1}\right)+\varphi\left(x_{n+1}\right)-\varphi(y)-p\left(x_{n+1}, x_{n+1}\right) \leq \varphi\left(x_{n}\right)-\varphi(y),
\end{aligned}
$$

which shows that $y \in A_{x_{n}}$, and so $A_{x_{n+1}} \subseteq A_{x_{n}}$.

For $x \in A_{x_{n+1}} \subseteq A_{x_{n}}$,

$$
\begin{aligned}
p\left(x_{n+1}, x\right) & \leq \varphi\left(x_{n+1}\right)-\varphi(x) \leq \inf \varphi\left(A_{x_{n}}\right)+\frac{1}{2^{n+1}}-\varphi(x) \\
& \leq \varphi(x)+\frac{1}{2^{n+1}}-\varphi(x)=\frac{1}{2^{n+1}} .
\end{aligned}
$$

For $m>n, x_{m} \in A_{x_{m-1}} \subseteq A_{x_{n}}$, so that $p\left(x_{n}, x_{m}\right)<1 / 2^{n}$, showing that the sequence $\left(x_{n}\right)$ is 0-Cauchy. It follows that there exists $z \in X$ with $p(z, z)=0$ such that

$$
\lim _{n} p\left(x_{n}, z\right)=0 \text {. }
$$

By Remark 7.35, $x_{n} \stackrel{q^{s}}{\rightarrow} z$. Since each set $A_{x_{n}}$ is $q^{s}$-closed and $x_{n+k} \in A_{x_{n+k-1}} \subseteq A_{x_{n}}$ for all $k \in \mathbb{N}$, it follows $z \in A_{x_{n}}$, for all $n \in \mathbb{N}$.

Also, the inequalities

$$
\begin{aligned}
p\left(x_{n}, f(z)\right) & \leq p\left(x_{n}, z\right)+p(z, f(z)) \leq \varphi\left(x_{n}\right)-\varphi(z)+\varphi(z)-\varphi(f(z)) \\
& \leq \varphi\left(x_{n}\right)-\varphi(f(z)),
\end{aligned}
$$

show that $f(z) \in \bigcap_{n=1}^{\infty} A_{x_{n}}$. Consequently, $p\left(x_{n}, f(z)\right)<1 / 2^{n}$ and, by the $q^{s}$-lsc of $p(\cdot, f(z))$,

$$
0 \leq p(z, f(z)) \leq \liminf _{n} p\left(x_{n}, f(z)\right) \leq \lim _{n} 1 / 2^{n}=0,
$$

so that $p(z, f(z))=0$. From

$$
p(f(z), f(z)) \leq p(f(z), z)+p(z, f(z))-p(z, z)=0,
$$

follows

$$
p(z, f(z))=p(z, z)=p(f(z), f(z))=0,
$$

which implies $f(z)=z$.

To prove the converse, suppose that the partial metric space $(X, p)$ is not 0 -complete. Then there exists a 0 -Cauchy sequence $\left(x_{n}\right)_{n=0}^{\infty}$ that is not properly convergent in $(X, p)$. Passing, if necessary, to a subsequence we can suppose further that the points $x_{n}$ are pairwise distinct and

$$
p\left(x_{n}, x_{n+1}\right)<\frac{1}{2^{n+1}} \quad \text { for all } n \in \mathbb{N}_{0}:=\mathbb{N} \cup\{0\} .
$$

Let

$$
A:=\left\{x_{n}: n \in \mathbb{N}_{0}\right\} .
$$

By Proposition 7.27 the sequence $\left(x_{n}\right)$ is $q^{s}$-Cauchy and not $q^{s}$-convergent, so it has no limit points, implying that the set $A$ is $q^{s}$-closed. 
Consider the functions $f: X \rightarrow X$ and $\varphi: X \rightarrow[0, \infty)$ given by

$$
f(x)=\left\{\begin{array}{ll}
x_{0} & \text { for } x \in X \backslash A, \\
x_{n+1} & \text { for } x=x_{n}, n \in \mathbb{N}_{0},
\end{array} \quad \text { and } \quad \varphi(x)= \begin{cases}p\left(x_{0}, x\right)+1 & \text { for } x \in X \backslash A, \\
1 / 2^{n} & \text { for } x=x_{n}, n \in \mathbb{N}_{0} .\end{cases}\right.
$$

It is obvious that $f$ has no fixed points.

I. The function $\varphi$ is $q^{s}$-lsc.

Let $\left(y_{n}\right)$ be a sequence in $X q^{s}$-convergent to some $y \in X$.

If $y \in X \backslash A$, then there exists $n_{0} \in \mathbb{N}$ such that $y_{n} \in X \backslash A$ for all $n \geq n_{0}$ (because the set $X \backslash A$ is $q^{s}$-open). Since $p\left(x_{0}, \cdot\right)$ is $q^{s}$-continuous (Proposition 7.27), it follows $\varphi(y)=\lim _{n} \varphi\left(y_{n}\right)$.

Suppose now that $y=x_{k}$ for some $k \in \mathbb{N}_{0}$ and denote by $\left(y_{m_{j}}\right)_{j \in \mathbb{N}}, m_{1}<m_{2}<\ldots$, the terms of the sequence $\left(y_{n}\right)$ that belong to $A$. If the set $\left\{m_{j}: j \in \mathbb{N}\right\}$ is infinite, then we must have $y_{m_{j}}=x_{k}, j \geq j_{0}$, for some $j_{0} \in \mathbb{N}$ (because $\left(x_{n}\right)$ has no convergent subsequences). Since $\varphi(x) \geq 1 \geq 2^{-k}$ for $x \in X \backslash A$, it follows $\inf \left\{\varphi\left(x_{i}\right): i \geq n\right\}=\varphi\left(x_{k}\right)$ for all $\mathrm{n}$, and $\operatorname{so} \liminf _{n} \varphi\left(y_{n}\right)=\sup _{n} \inf \left\{\varphi\left(x_{i}\right): i \geq n\right\}=\varphi\left(x_{k}\right)$.

If the set $\left\{m_{j}: j \in \mathbb{N}\right\}$ is finite, then there exists $n_{0} \in \mathbb{N}$ such that $y_{n} \in X \backslash A$ for all $n \geq n_{0}$. This implies

$$
\varphi\left(x_{k}\right)=\frac{1}{2^{k}} \leq 1 \leq \lim _{n}\left[p\left(x_{0}, y_{n}\right)+1\right]=\lim _{n} \varphi\left(y_{n}\right) .
$$

Consequently $\varphi(y) \leq \liminf _{n} \varphi\left(y_{n}\right)$ in both cases.

II. $f$ is a Caristi mapping with respect to $\varphi$.

Indeed, if $x \in X \backslash A$, then $f(x)=x_{0}$ and

$$
p(x, f(x))=p\left(x, x_{0}\right)=\varphi(x)-1=\varphi(x)-\varphi(f(x)) .
$$

If $x=x_{k}$ for some $k \in \mathbb{N}_{0}$, then $f\left(x_{k}\right)=x_{k+1}$ and, by (7.29),

$$
\begin{aligned}
p\left(x_{k}, f\left(x_{k}\right)\right) & =p\left(x_{k}, x_{k+1}\right)<\frac{1}{2^{k+1}} \\
& =\frac{1}{2^{k}}-\frac{1}{2^{k+1}}=\varphi\left(x_{k}\right)-\varphi\left(f\left(x_{k}\right)\right) .
\end{aligned}
$$

Consequently, $f$ is a $q^{s}$-Caristi mapping without fixed points.

Remark 7.39. Caristi-type fixed point theorems in complete partial metric spaces were also proved by Karapinar et al. in [26] and [103]. Since a complete partial metric space is 0 -complete, but the converse is not true (see [170]), these results follow from those proved by Romaguera 170

Another definition of Caristi condition in partial metric spaces was given by Acar, Altun and Romaguera 3. A mapping $f: X \rightarrow X$ is called AR-Caristi if

$$
\left(\operatorname{AR}-\operatorname{Car}_{\varphi}\right) \quad p(x, f(x)) \leq p(x, x)+\varphi(x)-\varphi(f(x)),
$$

for some $q^{s}$-lsc bounded from below function $\varphi: X \rightarrow \mathbb{R}$.

Theorem 7.40 (Acar, Altun and Romaguera [3]). A partial metric space $(X, p)$ is complete if and only if every AR-Caristi mapping on $X$ has a fixed point.

Proof. Suppose that $(X, p)$ is complete. Let $f: X \rightarrow X$ be a mapping satisfying the condition (AR-Car $\varphi$ for some $q^{s}$-lsc bounded from below function $\varphi: X \rightarrow \mathbb{R}$. By 
Proposition 7.27 the function $\beta: X \rightarrow[0, \infty)$ given by $\beta(x)=p(x, x), x \in X$, is $q^{s}$ continuous, so that the function $\psi:=\beta+2 \varphi$ is $q^{s}$-lsc and bounded from below (by $2 \inf \varphi(X))$.

Putting $\varphi=2^{-1}(\psi-\beta)$ in (AR-Car ${ }_{\varphi}$ and taking into account the definition (7.22) of the metric $q^{s}$ associated to the partial metric $p$, one obtains

$$
q^{s}(x, f(x)) \leq \psi(x)-\psi(f(x)) .
$$

Since, by Proposition 7.27 the metric space $\left(X, q^{s}\right)$ is complete, we can apply Caristi's fixed point theorem (Theorem 2.77) to the mapping $f$ and the $q^{s}$-lsc function $\psi$ to conclude that $f$ has a fixed point.

The proof of the converse follows the same line as that of the corresponding implication in Theorem 7.38 .

Suppose that $\left(x_{n}\right)_{n \in \mathbb{N}_{0}}\left(\mathbb{N}_{0}=\{0,1,2, \ldots\}\right)$ is a Cauchy sequence in $(X, p)$ which is not convergent. Passing to a subsequence, if necessary, we can suppose further that

$$
p\left(x_{n}, x_{n+1}\right)-p\left(x_{n}, x_{n}\right)<\frac{1}{2^{n+1}},
$$

for all $n \in \mathbb{N}_{0}$ (see Remark 7.28). It follows that the set

$$
A:=\left\{x_{n}: n \in \mathbb{N}_{0}\right\} .
$$

is $q^{s}$-closed in $\left(X, q^{s}\right)$.

Define the mappings $f: X \rightarrow X$ and $\varphi: X \rightarrow[0, \infty)$ by the formulae (7.30). Then $\varphi$ is $q^{s}$-lsc. It is obvious that the mapping $f$ has no fixed points, so it remains to show that it satisfies the condition (AR-Car $\left.{ }_{\varphi}\right)$.

For $x \in X \backslash A$,

$$
\begin{aligned}
p(x, f(x)) & =p\left(x, x_{0}\right)=\varphi(x)-\varphi(f(x)) \\
& \leq p(x, x)+\varphi(x)-\varphi(f(x)),
\end{aligned}
$$

while for $x=x_{n} \in A$,

$$
\begin{aligned}
p\left(x_{n}, f\left(x_{n}\right)\right) & =p\left(x_{n}, x_{n+1}\right)<p\left(x_{n}, x_{n+1}\right)+\frac{1}{2^{n+1}} \\
& =p\left(x_{n}, x_{n}\right)+\varphi\left(x_{n}\right)-\varphi\left(f\left(x_{n}\right)\right) .
\end{aligned}
$$

Remark 7.41. One can think to use the relations

$$
\psi=\beta+2 \varphi \Longleftrightarrow \varphi=\frac{1}{2}(\psi-\beta),
$$

in the proof of the converse. Indeed if $(X, p)$ is not complete, then $\left(X, q^{s}\right)$ is not complete (see Proposition 17.27), so, by Corollary 2.9, there exists a mapping $f: X \rightarrow X$ without fixed points which satisfies (7.32) for some $q^{s}$-lsc bounded from below function $\psi: X \rightarrow \mathbb{R}$. The function $\varphi=\frac{1}{2}(\psi-\beta)$ is $q^{s}$-lsc (because $\beta$ is $q^{s}$-continuous) and replacing $\psi$ by $\beta+2 \varphi$ in (17.32) one obtains (AR-Car ${ }_{\varphi}$ ).

Unfortunately, it is not sure that the function $\varphi=\frac{1}{2}(\psi-\beta)$ is bounded form below, in order to obtain a contradiction.

Remark 7.42. Caristi's Fixed Point Theorem for set-valued mappings on partial metric spaces is discussed in a recent paper by Alsiary and Latif [9]. 
7.8. Ekeland Variational Principle (EkVP) in partial metric spaces. In this subsection we shall show that in partial metric spaces Caristi's FPT is also equivalent to weak Ekeland principle.

Theorem 7.43 (Ekeland Variational Principle - weak form $(\mathrm{wEk}))$. Let $(X, p)$ be a 0 complete partial metric space and $\varphi: X \rightarrow \mathbb{R} \cup\{+\infty\} a q^{s}$-lsc bounded below proper function. Then for every $\varepsilon>0$ there exists $x_{\varepsilon} \in X$ such that

$$
\forall x \in X \backslash\left\{x_{\varepsilon}\right\}, \varphi\left(x_{\varepsilon}\right)<\varphi(x)+\varepsilon p\left(x, x_{\varepsilon}\right) .
$$

Proof. Suppose on the contrary that there exists $\varepsilon>0$ such that

$$
\forall x \in X, \exists y_{x} \in X \backslash\{x\} \text { with } \varphi(x) \geq \varphi\left(y_{x}\right)+\varepsilon p\left(x, y_{x}\right) .
$$

Consider a point $x_{0} \in X$ such that $\varphi\left(x_{0}\right) \leq \inf \varphi(X)+\varepsilon$ and let

$$
Y:=\left\{x \in X: \varphi(x)+\varepsilon p\left(x_{0}, x\right) \leq \varphi\left(x_{0}\right)+\varepsilon p\left(x_{0}, x_{0}\right)\right\} .
$$

By Proposition 7.27, the function $p\left(x_{0}, \cdot\right)$ is $q^{s}$-continuous, hence the function $\varphi(\cdot)+$ $\varepsilon p\left(x_{0}, \cdot\right)$ is $q^{s}$-lsc. Consequently, the set $Y$ is $p^{s}$-closed, and so 0 -complete. Indeed if $\left(x_{n}\right)$ is a 0 -Cauchy sequence in $Y$, then it has a $\tau_{p}$-limit $x \in X$ such that $p(x, x)=0$. But this implies $x_{n} \stackrel{q^{s}}{\rightarrow} x$ (see Remark 7.35) and so $x \in Y$. Also $Y \neq \emptyset$ because $x_{0} \in Y$ and $\varphi$ is finite on $Y$ (i.e. $\varphi(x) \in \mathbb{R}$ for all $x \in Y$ ).

Observe that the element $y_{x}$ given by (7.35) belongs to $Y$ for every $x \in Y$. Indeed, if $x \in Y$, then

$$
\begin{aligned}
\varphi\left(y_{x}\right)+\varepsilon p\left(x_{0}, y_{x}\right) & \leq \varphi(x)-\varepsilon p\left(x, y_{x}\right)+\varepsilon p\left(x_{0}, y_{x}\right) \\
& \leq \varphi\left(x_{0}\right)+\varepsilon p\left(x_{0}, x_{0}\right)+\varepsilon\left[p\left(x_{0}, y_{x}\right)-p\left(x_{0}, x\right)-p\left(x, y_{x}\right)\right] \\
& \leq \varphi\left(x_{0}\right)+\varepsilon p\left(x_{0}, x_{0}\right),
\end{aligned}
$$

because $p\left(x_{0}, y_{x}\right)-p\left(x_{0}, x\right)-p\left(x, y_{x}\right) \leq 0$. This last inequality follows from

$$
p\left(x_{0}, y_{x}\right) \leq p\left(x_{0}, x\right)+p\left(x, y_{x}\right)-p(x, x) \leq p\left(x_{0}, x\right)+p\left(x, y_{x}\right) .
$$

Put now $\tilde{\varphi}:=\left.\varepsilon^{-1} \varphi\right|_{Y}: Y \rightarrow \mathbb{R}$ and let $f: Y \rightarrow Y$ be defined by $f(x)=y_{x}$, where, for $x \in Y, y_{x} \neq x$ is the element of $Y$ satisfying (7.35).

Then the inequality (7.35) is equivalent to

$$
p(x, f(x)) \leq \tilde{\varphi}(x)-\tilde{\varphi}(f(x)) \quad x \in Y,
$$

which shows that $f$ is a Caristi mapping with respect to $\tilde{\varphi}$. Since $f$ has no fixed points, this is in contradiction to Caristi's fixed point theorem (Theorem 7.38)

We show now that the converse implication also holds.

Proposition 7.44. Ekeland's Variational Principle in its weak form (Theorem 7.43) implies Caristi's Fixed Point Theorem (Theorem 7.38).

Proof. Let $(X, p)$ be a 0-complete partial metric space, $\varphi: X \rightarrow \mathbb{R}$ a $q^{s}$-lsc bounded from below function and $f: X \rightarrow X$ a Caristi mapping with respect to $\varphi$. By Theorem 7.43 applied to $\varphi$ for $\varepsilon=1$ there exists a point $x_{1} \in X$ such that

$$
\varphi\left(x_{1}\right)<\varphi(x)+p\left(x_{1}, x\right),
$$

for all $x \in X \backslash\left\{x_{1}\right\}$. Supposing $f\left(x_{1}\right) \neq x_{1}$, we can take $x=f\left(x_{1}\right)$ in the above inequality to obtain

$$
p\left(x_{1}, f\left(x_{1}\right)\right)>\varphi\left(x_{1}\right)-\varphi\left(f\left(x_{1}\right)\right),
$$

in contradiction to the inequality $\operatorname{Car}_{\varphi}$ satisfied by $f$. 
Consequently $f\left(x_{1}\right)=x_{1}$, that is $x_{1}$ is a fixed point of $f$.

Remark 7.45. It follows that the validity of Ekeland's Variational Principle in its weak form, as given in Theorem 7.43 , is also equivalent to the 0 -completeness of the partial metric space $(X, p)$.

We shall present now the version of Ekeland Variational Principle that can be obtained from Theorem 7.40 .

Theorem 7.46 (Ekeland Variational Principle 2 - weak form (wEk2)). Let $(X, p)$ be a complete partial metric space and $\varphi: X \rightarrow \mathbb{R} \cup\{\infty\}$ a $q^{s}$-lsc bounded below proper function. Then for every $\varepsilon>0$ there exists $x_{\varepsilon} \in X$ such that

$$
\forall x \in X \backslash\left\{x_{\varepsilon}\right\}, \varphi\left(x_{\varepsilon}\right)+\varepsilon p\left(x_{\varepsilon}, x_{\varepsilon}\right)<\varphi(x)+\varepsilon p\left(x, x_{\varepsilon}\right) .
$$

Proof. Suppose, by contradiction, that there exists an $\varepsilon>0$ such that

$$
\forall x \in X, \exists y_{x} \in X \backslash\{x\} \text { with } \varphi(x)+\varepsilon p(x, x) \geq \varphi\left(y_{x}\right)+\varepsilon p\left(x, y_{x}\right),
$$

and let $x_{0} \in X$ be such that $\varphi\left(x_{0}\right) \leq \varepsilon+\inf \varphi(X)$.

To get rid of the points where $\varphi$ takes the value $+\infty$, consider again the set $Y$ given by (7.36). Then $Y$ is nonempty $\left(x_{0} \in Y\right)$ and $q^{s}$-closed and so complete with respect to the partial metric $p$. Indeed if $\left(x_{n}\right)$ is a Cauchy sequence in $(X, p)$ then, by the definition of the completeness, it converges properly to some $x \in X$. By Proposition [7.27, $\left(x_{n}\right)$ is $q^{s}$-convergent to $x$ and so $x \in Y$.

Observe that $x \in Y$ implies that the element $y_{x}$ given by (7.38) also belongs to $Y$. Indeed, if $x \in Y$, then

$$
\begin{aligned}
\varphi\left(y_{x}\right)+\varepsilon p\left(x_{0}, y_{x}\right) & \leq \varphi(x)+\varepsilon\left[p\left(x_{0}, y_{x}\right)-p\left(x, y_{x}\right)+p(x, x)\right] \\
& \leq \varphi\left(x_{0}\right)+\varepsilon\left[p\left(x_{0}, x_{0}\right)-p\left(x_{0}, x\right)+p\left(x_{0}, y_{x}\right)-p\left(x, y_{x}\right)+p(x, x)\right] \\
& \leq \varphi\left(x_{0}\right)+\varepsilon p\left(x_{0}, x_{0}\right),
\end{aligned}
$$

because

$p\left(x_{0}, y_{x}\right)-p\left(x_{0}, x\right)-p\left(x, y_{x}\right)+p(x, x) \leq 0 \Longleftrightarrow p\left(x_{0}, y_{x}\right)+p(x, x) \leq p\left(x_{0}, x\right)+p\left(x, y_{x}\right)$, and the last inequality is true, by the triangle inequality (PM4) from Definition 7.1 .

Taking again $\tilde{\varphi}=\left.\varepsilon^{-1} \varphi\right|_{Y}$ and $f: Y \rightarrow Y$ defined by $f(x)=y_{x}$, where for $x \in Y$ the element $y_{x} \in Y$ is given by (7.38), the function $\tilde{\varphi}$ is $q^{s}$-lsc and $f$ is a mapping on $Y$ without fixed points, satisfying (AR-Car $\bar{\varphi}$ ) for $\varphi=\tilde{\varphi}$.

The converse implication holds in this case too. The proof is similar to that of Proposition 7.44 .

Proposition 7.47. Ekeland's Variational Principle in its weak form, as given in Theorem 7.46. implies Caristi's Fixed Point Theorem, as given in Theorem 7.40.

Proof. Let $(X, p)$ be a complete partial metric space, $\varphi: X \rightarrow \mathbb{R}$ a $q^{s}$-lsc bounded from below function and $f: X \rightarrow X$ a mapping satisfying (AR-Car $\varphi$. Applying Theorem 7.43 to $\varphi$ for $\varepsilon=1$ it follows the existence of a point $x_{1} \in X$ such that

$$
\varphi\left(x_{1}\right)+p\left(x_{1}, x_{1}\right)<\varphi(x)+p\left(x_{1}, x\right)
$$

for all $x \in X \backslash\left\{x_{1}\right\}$. Supposing $f\left(x_{1}\right) \neq x_{1}$, we can take $x=f\left(x_{1}\right)$ in the above inequality to obtain

$$
p\left(x_{1}, f\left(x_{1}\right)\right)>p\left(x_{1}, x_{1}\right)+\varphi\left(x_{1}\right)-\varphi\left(f\left(x_{1}\right)\right),
$$

in contradiction to the inequality $\mathrm{AR}-\mathrm{Car}_{\varphi}$ satisfied by $f$. 
Consequently $f\left(x_{1}\right)=x_{1}$, that is $x_{1}$ is a fixed point of $f$.

Remark 7.48. It follows that the validity of Ekeland's Variational Principle in its weak form, as given in Theorem 7.46, is equivalent to the completeness of the partial metric space $(X, p)$.

Remark 7.49. A version of Ekeland Variational Principle in partial metric spaces was proved by Aydi, Karapinar and Vetro [27].

7.9. Dislocated metric spaces. This class of spaces was considered by Hitzler and Seda [80 in connection with some problems in logic programming. A dislocated metric on a set $X$ is a function $\rho: X \times X \rightarrow \mathbb{R}_{+}$satisfying the conditions:

$$
\begin{array}{ll}
\text { (DM1) } & \rho(x, y)=0 \Rightarrow x=y \\
\text { (DM2) } & \rho(x, y)=\rho(y, x) \\
\text { (DM3) } & \rho(x, y) \leq \rho(x, z)+\rho(z, y),
\end{array}
$$

for all $x, y, z \in X$. If $\rho$ satisfies only (DM1) and (DM3), then it is called a dislocated quasimetric. The pair $(X, \rho)$ is called a dislocated metric (resp. a dislocated quasi-metric) space.

These spaces are close to partial metric spaces (in this case it is also possible that $\rho(x, x)>0$ for some $x \in X)$, with the exception that a dislocated metric satisfies the usual triangle inequality (DM3) instead of the inequality (PM4) from Definition 7.1. In fact, any partial metric is a dislocated metric.

For $x \in X$ and $r>0$ the open ball $B(x, r)$ is defined by $B(x, r)=\{y \in X: \rho(x, y)<r\}$.

Hitzler and Seda [80] defined a kind of topology on a dislocated metric space $(X, \rho)$ in the following way. Instead of the membership relation $\in$ they considered a relation $\prec$ in $X \times 2^{X}$, defined for $(x, A) \in X \times 2^{X}$ by

$$
x \prec A \Longleftrightarrow \exists \varepsilon>0 \text { such that } B(x, \varepsilon) \subseteq A .
$$

The d-neighborhood system $\mathcal{V}(x)$ of a point $x \in X$ is defined by the condition

$$
V \in \mathcal{V}(x) \Longleftrightarrow V \subseteq X \quad \text { and } \quad x \prec V \text {. }
$$

(Here "d-" comes from "dislocated-").

The neighborhood axioms are satisfied with the relation $\prec$ instead of $\in$.

(V1) $V \in \mathcal{V}(x) \Longrightarrow x \prec V$;

(V2) $V \in \mathcal{V}(x)$ and $V \subseteq U \Longrightarrow U \in \mathcal{V}(x)$;

(V3) $U, V \in \mathcal{V}(x) \Longrightarrow U \cap V \in \mathcal{V}(x)$;

(V4) $V \in \mathcal{V}(x) \Longrightarrow \exists W \in \mathcal{V}(x), W \subseteq V$ such that $V \in \mathcal{V}(y)$ for all $y \prec W$.

It is easy to check the validity of these properties. As a sample, let us check (V4). For $V \in \mathcal{V}(x)$ let $\varepsilon>0$ be such that $B(x, \varepsilon) \subseteq V$. If $y \prec B(x, \varepsilon)$, then there exists $\varepsilon^{\prime}>0$ such that $B\left(y, \varepsilon^{\prime}\right) \subseteq B(x, \varepsilon) \subseteq V$, so that $V \in \mathcal{V}(y)$. It follows that we can take $W=B(x, \varepsilon)$.

The so defined "neighborhood system" is not a proper neighborhood system (i.e., with respect to the relation $\in$ ), because the relation $x \in V$ is not always satisfied - it is not sure that $x \in B(x, \varepsilon)$ and further, the ball $B(x, \varepsilon)$ could be empty for some $\varepsilon$.

Example 7.50. Let $X$ be a set of cardinality at least 2. Define $\rho(x, x)=1$ and $\rho(x, y)=2$ if $x \neq y$, for all $x, y \in X$. Then $B(x, \varepsilon)=\emptyset$ for $0<\varepsilon \leq 1$, implying that every subset of $X$ (including the empty set) is a d-neighborhood of $x$. 
In fact the following properties hold.

Proposition 7.51 ([80], Proposition 3.2). Let $(X, \rho)$ be a dislocated metric space.

(1) The following conditions are equivalent:

(i) $\rho$ is a metric.

(ii) $\rho(x, x)=0$ for all $x \in X$.

(iii) $B(x, \varepsilon) \neq \emptyset$ for all $x \in X$ and $\varepsilon>0$.

(2) The subset $\operatorname{ker} \rho:=\{x \in X: \rho(x, x)=0\}$ is a metric space with respect to $\rho$.

A sequence $\left(x_{n}\right)$ in $X$ is called d-convergent to $x \in X$ if

$$
\forall V \in \mathcal{V}(x), \exists n_{0} \in \mathbb{N} \text { such that } x_{n} \in V \text { for all } n \geq n_{0} .
$$

The sequence $\left(x_{n}\right)$ in $X$ is called $\rho$-convergent to $x$ if $\lim _{n \rightarrow \infty} \rho\left(x, x_{n}\right)=0$.

Remark 7.52. Note again that this type of convergence is not a proper convergence. For instance, if $\rho(x, x)>0$, then the constant sequence $x_{n}=x, n \in \mathbb{N}$, is not $\rho$-convergent to $x$.

The following property holds.

Proposition 7.53 ([80], Proposition 3.9). Let $(X, \rho)$ be a dislocated metric space. A sequence $\left(x_{n}\right)$ in $X$ is $\rho$-convergent to $x \in X$ if and only if it is $d$-convergent to $x$.

Proof. Suppose that $\left(x_{n}\right)$ is d-convergent to $x$. For $\varepsilon>0, B(x, \varepsilon)$ is a d-neighborhood of $x$, so there exists $n_{0} \in \mathbb{N}$ such that $x_{n} \in B(x, \varepsilon) \Longleftrightarrow \rho\left(x, x_{n}\right)<\varepsilon$, for all $n \geq n_{0}$, showing that $\rho\left(x, x_{n}\right) \rightarrow 0$.

Suppose now that $\rho\left(x, x_{n}\right) \rightarrow 0$. For $V \in \mathcal{V}(x)$ let $\varepsilon>0$ be such that $B(x, \varepsilon) \subseteq V$. By hypothesis there exists $n_{0} \in \mathbb{N}$ such that $\rho\left(x, x_{n}\right)<\varepsilon$ for all $n \geq n_{0}$. It follows $x_{n} \in B(x, \varepsilon) \subseteq V$ for all $n \geq n_{0}$.

A sequence $\left(x_{n}\right)$ in $X$ is called $\rho$-Cauchy if for every $\varepsilon>0$ there exists $n_{0} \in \mathbb{N}$ such that $\rho\left(x_{n}, x_{m}\right)<\varepsilon$ for all $m, n \geq n_{0}$. The dislocated metric space $(X, \rho)$ is called complete if every Cauchy sequence is $\rho$-convergent. Hitzler and Seda [80, Theorem 2.7] proved that Banach's contraction principle holds in complete dislocated metric spaces.

Pasicki [161] defined a topology $\tau_{\rho}$ on a dislocated metric space $(X, \rho)$ in the following way. The family of subsets $\{B(x, r): x \in X, r>0\}$ satisfies $X=\bigcup\{B(x, r): x \in X, r>$ $0\}$, so it is a subbase for a topology $\tau_{\rho}$ on $X$ (see Kelley [109, Theorem 12, p. 47]).

It follows that a subset $U$ of $X$ is a neighborhood of $x \in X$ if and only if

$\exists n \in \mathbb{N}, \exists y_{1}, \ldots, y_{n} \in X, \exists r_{1}, \ldots, r_{n}>0$, such that $x \in B\left(y_{1}, r_{1}\right) \cap \cdots \cap B\left(y_{n}, r_{n}\right) \subseteq U$.

Denote by $\mathcal{U}_{\rho}(x)$ the neighborhood system of a point $x \in X$ with respect to $\tau_{\rho}$.

Remark 7.54. Let $\left(x_{n}\right)$ be a sequence in a dislocated metric space $(X, \rho)$ and $x \in X$. If $\lim _{n} \rho\left(x, x_{n}\right)=0$, then the sequence $\left(x_{n}\right)$ is $\tau_{\rho}$-convergent to $x \in X$.

Indeed, for any $\tau_{\rho}$-neighborhood $U$ of $x$ there exists $y \in X$ and $\varepsilon>0$ such that $x \in B(y, \varepsilon) \subseteq U$. Then $\varepsilon-\rho(x, y)>0$ so that, by hypothesis, there exists $n_{0} \in \mathbb{N}$ such that $\rho\left(x, x_{n}\right)<\varepsilon-\rho(x, y)$ for all $n \geq n_{0}$. It follows

$$
\rho\left(y, x_{n}\right) \leq \rho(y, x)+\rho\left(x, x_{n}\right)<\rho(x, y)+\varepsilon-\rho(x, y)=\varepsilon,
$$

that is $x_{n} \in B(y, \varepsilon) \subseteq U$ for all $n \geq n_{0}$, showing that $\left(x_{n}\right)$ is $\tau_{\rho}$-convergent to $x$.

Remark 7.55. I don't know a characterization of the $\tau_{\rho}$-convergence in terms of the sequence $\left(\rho\left(x, x_{n}\right)\right)_{n \in \mathbb{N}}$. 
Apparently unaware of Hitzler and Seda paper [80, Amini-Harandi [15] defined dislocated metric spaces calling them metric-like spaces. He defined the balls by analogy with partial metric spaces:

$$
\tilde{B}(x, \varepsilon)=\{y \in X:|\rho(x, y)-\rho(x, x)|<\varepsilon\} .
$$

The family of balls $\tilde{B}(x, r), x \in X, \varepsilon>0$ form the base of a topology $\tilde{\tau}_{\rho}$ on the dislocated metric space $(X, \rho)$.

A sequence $\left(x_{n}\right)$ in $X$ is $\tilde{\tau}_{\rho}$-convergent to $x \in X$ if and only if $\lim _{n \rightarrow \infty} \rho\left(x, x_{n}\right)=\rho(x, x)$.

A sequence $\left(x_{n}\right)$ in $X$ is called Cauchy if there exists the $\operatorname{limit}_{m, n \rightarrow \infty} \rho\left(x_{n}, x_{m}\right) \in \mathbb{R}$. The space $(X, \rho)$ is called complete if for every Cauchy sequence $\left(x_{n}\right)$ in $X$ there exists $x \in X$ such that

$$
\lim _{n \rightarrow \infty} \rho\left(x, x_{n}\right)=\rho(x, x)=\lim _{m, n \rightarrow \infty} \rho\left(x_{m}, x_{n}\right),
$$

(compare with Subsection 7.2, Definition 7.12).

The paper [80] contains some fixed point theorems in complete dislocated metric spaces. The same approach is adopted in the paper [105] (and possibly in other papers).

Remark 7.56. In fact, in a preliminary version of the paper [161, Pasicki called these spaces near metric spaces. After the reviewer draw his attention to Hitzler and Seda paper, he changed to dislocated metric spaces. There are a lot of papers dealing with fixed point results in dislocated metric spaces (or in metric like spaces), as it can be seen by a simple search on MathSciNet, ZbMATH or ScholarGoogle. I don't know if there are some converse results - i.e. completeness implied by the validity of some fixed point results.

7.10. Other generalized metric spaces. In this subsection we shall present some completeness results in other classes of generalized metric spaces: dislocated metric spaces, $w$-spaces and $\tau$-spaces. Good surveys of various generalizations of metric spaces are given in the papers by Ansari [21, Berinde and Choban [36], and in the books [57], [112] and [179].

\section{$w$-distances}

This notion was introduced by Kada et al. [102]. Let $(X, \rho)$ be a metric space. A mapping $p: X \times X \rightarrow \mathbb{R}_{+}$is called a $w$-distance if, for all $x, y, z \in X$,

$$
p(x, y) \leq p(x, z)+p(z, y)
$$

(w3) $\forall \varepsilon>0, \exists \delta>0$ such that $p(x, y)<\delta$ and $p(x, z)<\delta$ implies $p(y, z)<\varepsilon$.

\section{$\tau$-distances}

A more involved notion was introduced by Suzuki [191. Let $(X, \rho)$ be a metric space and $\eta: X \times \mathbb{R}_{+} \rightarrow \mathbb{R}_{+}$. A mapping $p: X \times X \rightarrow \mathbb{R}_{+}$is called a $\tau$-distance if

$(\tau 1) p(x, y) \leq p(x, z)+p(z, y)$ for all $x, y, z \in X$

$(\tau 2)$ for every $x \in X$ the function $\eta(x, \cdot)$ is concave and continuous, $\eta(x, 0)=0$ and $\eta(x, t) \geq t$ for all $(x, t) \in X \times \mathbb{R}_{+}$;

$(\tau 3) \lim _{n} x_{n}=x$ and $\lim _{n}\left(\sup _{m \geq n} \eta\left(z_{n}, p\left(z_{n}, x_{m}\right)\right)\right)=0$ imply $p(w, x) \leq \liminf _{n} p\left(w, x_{n}\right)$ for all $w \in X$

$(\tau 4) \lim _{n}\left(\sup _{m \geq n} p\left(x_{n}, y_{m}\right)\right)=0$ and $\lim _{n} \eta\left(x_{n}, t_{n}\right)=0$ imply $\lim _{n} \eta\left(y_{n}, t_{n}\right)=0$; 
$(\tau 5) \lim _{n} \eta\left(z_{n}, p\left(z_{n}, x_{n}\right)\right)=0$ and $\lim _{n} \eta\left(z_{n}, p\left(z_{n}, y_{n}\right)\right)=0$ imply $\lim _{n} \rho\left(x_{n}, y_{n}\right)=0$.

Remark 7.57. It was shown in [191] that $(\tau 2)$ can be replaced by

$\left(\tau 2^{\prime}\right) \quad$ for every $x \in X$ the function $\eta(x, \cdot)$ is increasing and $\inf _{t>0} \eta(x, t)=0$.

Lin and $\mathrm{Du}[128,130$, propose a slightly simplified version of a $\tau$-function.

Let $(X, \rho)$ be a metric space. A mapping $p: X \times X \rightarrow \mathbb{R}_{+}$is called a $(\mathrm{LD} \tau)$-distance if

$(\mathrm{LD}-\tau 1) p(x, y) \leq p(x, z)+p(z, y)$ for all $x, y, z \in X$;

(LD- $\tau 2)$ for every $x \in X$ and every sequence $\left(y_{n}\right)$ in $X$ converging to some $y \in X$, if for some $M>0, p\left(x, y_{n}\right) \leq M$, for all $n$, then $p(x, y) \leq M$;

$(\mathrm{LD}-\tau 3)$ if $\left(x_{n}\right)$ and $\left(y_{n}\right)$ are sequences in $X$ such that $\lim _{n}\left(\sup _{m \geq n} p\left(x_{n}, x_{m}\right)\right)=0$ and $\lim _{n} p\left(x_{n}, y_{n}\right)=0$, then $\lim _{n} \rho\left(x_{n}, y_{n}\right)=0$

(LD- $\tau 4)$ for all $x, y, z \in X, p(x, y)=p(x, z)=0$ implies $y=z$.

\section{Remark 7.58.}

1. If, for every $x \in X, p(x, \cdot)$ is lsc, then condition (LD- $\tau 2)$ is satisfied.

2. If $p$ satisfies (LD- $\tau 3$ ), then every sequence $\left(x_{n}\right)$ in $X$ satisfying

$$
\lim _{n}\left(\sup _{m \geq n} p\left(x_{n}, x_{m}\right)\right)=0
$$

is a Cauchy sequence.

Lin and Du proved in [128, 130] variational principles of Ekeland type for this kind of function, and for the $w$-distance in [129].

\section{Tataru distance}

This was defined by Tataru [210] in the following way. Let $X$ be a subset of a Banach space $E$. A family $\left\{T(t): t \in \mathbb{R}_{+}\right\}$of mappings on $X$ is called a strongly continuous semigroup of nonexpansive mappings on $X$ if

(Sg1) for every $t \in \mathbb{R}_{+}, T(t)$ is a nonexpansive mapping on $X$;

(Sg2) $T(0) x=x$ for all $x \in X$;

(Sg3) $T(s+t)=T(s) T(t)$ for all $s, t \in \mathbb{R}_{+}$;

(Sg4) for each $x \in X$ the mapping $T(\cdot) x: \mathbb{R}_{+} \rightarrow X$ is continuous.

The Tataru distance corresponding to a strongly continuous semigroup $\left\{T(t): t \in \mathbb{R}_{+}\right\}$ of nonexpansive mappings on $X$ is defined for $x, y \in X$ by

$$
p(x, y)=\inf \left\{t+\|T(t) x-y\|: t \in \mathbb{R}_{+}\right\} .
$$

It was shown by Suzuki, [191] and [197, that any $w$-distance is a $\tau$-distance, but the converse does not hold - for instance, the Tataru distance is a $w$-distance but not a $\tau$ distance. The paper [197] contains many examples of $w$-distances and $\tau$-distances, other $\tau$-distances which are not $w$-distances, and conditions under which the Tataru distance is a $\tau$-distance.

Various fixed point results, Ekeland-type principles and completeness for $\tau$-distances were proved by Suzuki in [191, 192, 193, 194, 198, 199, 201].

Fixed points for contractions and completeness results in quasi-metric spaces endowed with a $w$-distance were proved by Alegre et al. [5], for single-valued maps, and by Marín 
et al. [137], for set-valued ones. Similar results in the case of partial metric spaces were obtained by Altun and Romaguera [10].

A mapping $f$ on a metric space $(X, \rho)$ for which there exist a $w$-distance $p$ on $X$ and a number $\alpha \in[0,1)$ such that

$$
p\left(f(x), f\left(x^{\prime}\right)\right) \leq \alpha p(x, y) \text { for all } x, x^{\prime} \in X,
$$

is called weakly contractive (or a weak contraction). In the case of a set-valued mapping $F: X \rightrightarrows X$, the condition (7.41) is replaced by

$$
\forall x, x^{\prime} \in X, \exists y \in F(x), y^{\prime} \in F\left(x^{\prime}\right) \text {, such that } p\left(y, y^{\prime}\right) \leq \alpha p(x, y) .
$$

Direct and converse fixed point results involving completeness for weakly contractive mappings and for other types of mappings (e.g. Kannan maps) on metric spaces endowed with a $w$-distance were proved in [46, 87, 102, 184, 202, 204, 206] (see also the books [112] and [205]). For instance, in [202] it is proved that a metric space $X$ is complete if and only if every weakly contractive mapping on $X$ has a fixed point. Also, the result of Borwein [39] (see Corollary 1.26), on the completeness of convex subsets of normed spaces on which every contraction has a fixed point, is rediscovered.

\section{Branciari's distance - generalized metric spaces}

Branciari [41] (see [180] for some corrections) introduced a new class of spaces, called generalized metric spaces, in the following way. A function $d: X \times X \rightarrow \mathbb{R}_{+}$, where $X$ is a nonempty set, is called a generalized metric if the following conditions hold

(GM1) $d(x, y)=0 \Longleftrightarrow x=y$;

(GM2) $d(x, y)=d(y, x)$;

$($ GM3 $) d(x, y) \leq d(x, u)+d(u, v)+d(v, y)$,

for all pairwise distinct points $x, y, u, v \in X$. The generalized triangle inequality (GM3) causes several troubles concerning the topology of these spaces (it is not always Hausdorff and the distance function $d(\cdot, \cdot)$ is continuous only under a supplementary condition, see [112, Ch. 13]) and the completeness. Branciari loc. cit. proved a Banach Contraction Principle within this context (some flaws in the original proof are corrected in [112, Ch. $13])$.

Ghosh and Deb Ray [72] considered Suzuki's generalized contractions for these spaces and proved direct fixed point results as well as converse completeness results.

\section{Probabilistic metric spaces}

Completeness as well as relations between completeness and fixed point results in probabilistic metric spaces are explored in the papers [4], 8], [76], [83]. We do not enter into the details of this matter.

\section{APPENDIX - A PESSIMISTIC CONCLUSION}

In conclusion we quote from the review of the paper [160].

MR835839 (87m:54125) Park, Sehie; Rhoades, B. E. Comments on characterizations for metric completeness. Math. Japon. 31 (1986), no. 1, 95-97. 
There are many papers in which the completeness of a metric space is characterized by using a fixed point theorem. In the present paper the authors prove two very simple and general theorems which "encompass some previous as well as future theorems of this type".

(Reviewed by J. Matkowski)

Under these circumstances, it seems that the best we can hope to do in this domain is to prove some particular cases of these very general results.

Acknowledgement. This is an expanded version of a talk delivered at the International Conference on Nonlinear Operators, Differential Equations and Applications (ICNODEA 2015), Cluj-Napoca, Romania, July 14-17, 2015.

\section{REFERENCES}

[1] T. Abdeljawad, E. Karapınar, and K. Taş, Existence and uniqueness of a common fixed point on partial metric spaces, Appl. Math. Lett. 24 (2011), no. 11, 1900-1904. 70

[2] S. Abramsky and A. Jung, Domain theory, Handbook of logic in computer science, Vol. 3, Handb. Log. Comput. Sci., vol. 3, Oxford Univ. Press, New York, 1994, pp. 1-168. 50

[3] Ö. Acar, I. Altun, and S. Romaguera, Caristi's type mappings on complete partial metric spaces, Fixed Point Theory 14 (2013), no. 1, 3-9. 2. 72

[4] A. Aghajani and A. Razani, Some completeness theorems in the Menger probabilistic metric space, Appl. Sci. 10 (2008), 1-8. 80

[5] C. Alegre, J. Marín, and S. Romaguera, A fixed point theorem for generalized contractions involving $w$-distances on complete quasi-metric spaces, Fixed Point Theory Appl. (2014), 2014:40, 8 pp. 79

[6] M. A. Alghamdi, N. Shahzad, and O. Valero, On fixed point theory in partial metric spaces, Fixed Point Theory Appl. (2012), 2012:175, 25 pp. 62

[7] _ New results on the Baire partial quasi-metric space, fixed point theory and asymptotic complexity analysis for recursive programs, Fixed Point Theory Appl. (2014), 2014:14, 18 pp. 62

[8] M. Alimohammady, A. Esmaeli, and R. Saadati, Completeness results in probabilistic metric spaces, Chaos Solitons Fractals 39 (2009), no. 2, 765-769. 80

[9] T. Alsiary and A. Latif, Generalized Caristi fixed point results in partial metric spaces, J. Nonlinear Convex Anal. 16 (2015), no. 1, 119-125. 73

[10] I. Altun and S. Romaguera, Characterizations of partial metric completeness in terms of weakly contractive mappings having fixed point, Appl. Anal. Discrete Math. 6 (2012), no. 2, 247-256. 80

[11] I. Altun, F. Sola, and H. Simsek, Generalized contractions on partial metric spaces, Topology Appl. 157 (2010), no. 18, 2778-2785. 60.

[12] P. Amato, A method for reducing fixed-point problems to completeness problems and vice versa, Boll. Un. Mat. Ital. B (6) 3 (1984), no. 2, 463-476 (Italian). 14

[13] The completion classes of a metric space, Rend. Circ. Mat. Palermo (2) Suppl. (1986), no. 12, 157-168 (Italian), Second topology conference (Taormina, 1984). 14

[14] _ Some properties of completion classes for normed spaces, Note Mat. 13 (1993), no. 1, 123134. 14

[15] A. Amini-Harandi, Metric-like spaces, partial metric spaces and fixed points, Fixed Point Theory Appl. (2012), 2012:204, 10 pp. 78

[16] V. G. Angelov, Fixed point theorem in uniform spaces and applications, Czechoslovak Math. J. 37(112) (1987), no. 1, 19-33. 4

[17] - A converse to a contraction mapping theorem in uniform spaces, Nonlinear Anal. 12 (1988), no. 10, 989-996. 4

[18] - Corrigendum: "A converse to a contraction mapping theorem in uniform spaces" [Nonlinear Anal. 12 (1988), no. 10, 989-996; MR0962764 (89k:54101)], Nonlinear Anal. 23 (1994), no. 11, 1491. 4

[19] An extension of Kirk-Caristi theorem to uniform spaces, Antarct. J. Math. 1 (2004), no. 1, 47-51. 4 
[20] M. Anisiu and V. Anisiu, On the characterization of partial metric spaces and quasimetrics, Fixed Point Theory 17 (2016), no. 1, 37-46. 57

[21] Q. H. Ansari and L.-J. Lin, Ekeland-type variational principles and equilibrium problems, Topics in nonconvex optimization, Springer Optim. Appl., vol. 50, Springer, New York, 2011, pp. 147-174. 78

[22] A. V. Arutyunov, Caristi's condition and existence of a minimum of a lower bounded function in a metric space. Applications to the theory of coincidence points, Proc. Steklov Inst. Math. 291 (2015), no. 1, 24-37, Translation of Tr. Mat. Inst. Steklova 291 (2015), 30-44. 26] 27

[23] A. V. Arutyunov and B. D. Gel'man, The minimum of a functional in a metric space, and fixed points, Zh. Vychisl. Mat. Mat. Fiz. 49 (2009), no. 7, 1167-1174. 26

[24] A. V. Arutyunov and S. E. Zhukovskiy, Variational principles in analysis and existence of minimizers for functions on metric spaces, SIAM J. Optim. 29 (2019), no. 2, 994-1016. 26

[25] A. V. Arutyunov, E. S. Zhukovskiy, and S. E. Zhukovskiy, Caristi-like condition and the existence of minima of mappings in partially ordered spaces, J. Optim. Theory Appl. 180 (2019), no. 1, 48-61. 26

[26] H. Aydi, E. Karapınar, and P. Kumam, A note on 'Modified proof of Caristi's fixed point theorem on partial metric spaces, Journal of Inequalities and Applications 2013, 2013:210', J. Inequal. Appl. (2013), 2013:355, 3 pp. 72

[27] H. Aydi, E. Karapınar, and C. Vetro, On Ekeland's variational principle in partial metric spaces, Appl. Math. Inf. Sci. 9 (2015), no. 1, 257-262. 76

[28] A. C. Babu, A converse to a generalized Banach contraction principle, Publ. Inst. Math. (Beograd) (N.S.) 32(46) (1982), 5-6. 4

[29] S. Banach, Sur les opérations dans les ensembles abstraits et leur application aux équations intégrales., Fund. Math. 3 (1922), 133-181 (French). 2

[30] T. Q. Bao, S. Cobzaş, and A. Soubeyran, Variational principles and completeness in pseudoquasimetric spaces, Ann. Oper. Res. 269 (2018), no. 1-2, 53-79, doi.org/10.1007/s10479-016-2368-0. $39,41,42$

[31] T. Q. Bao, B. S. Mordukhovich, and A. Soubeyran, Fixed points and variational principles with applications to capability theory of wellbeing via variational rationality, Set-Valued Var. Anal. 23 (2015), no. 2, 375-398. 43

[32] - Variational analysis in psychological modeling, J. Optim. Theory Appl. 164 (2015), no. 1, 290-315. 43

[33] T. Q. Bao and A. Soubeyran, Variational analysis and applications to group dynamics, J. Optim. Theory Appl. 170 (2016), no. 2, 458-475. 39, 43

[34] T. Q. Bao and M. A. Théra, On extended versions of Dancs-Hegedüs-Medvegyev's fixed-point theorem, Optimization 66 (2017), no. 6, 875-887. 39

[35] V. Berinde and M. Choban, Remarks on some completeness conditions involved in several common fixed point theorems, Creat. Math. Inform. 19 (2010), no. 1, 1-10. 28

[36] - Generalized distances and their associate metrics. Impact on fixed point theory, Creat. Math. Inform. 22 (2013), no. 1, 23-32. 78

[37] C. Bessaga, On the converse of the Banach "fixed-point principle", Colloq. Math. 7 (1959), 41-43. 4

[38] F. Blanqui, A point on fixpoints in posets, arXiv: 1502.06021 (2014), 10 pp. 51

[39] J. M. Borwein, Completeness and the contraction principle, Proc. Amer. Math. Soc. 87 (1983), no. 2, 246-250. 8, 9, 12,80

[40] N. Bourbaki, Sur le théorème de Zorn, Arch. Math. (Basel) 2 (1949-1950), 434-437 (1951). 51

[41] A. Branciari, A fixed point theorem of Banach-Caccioppoli type on a class of generalized metric spaces, Publ. Math. Debrecen 57 (2000), no. 1-2, 31-37. 80

[42] M. Bukatin, R. Kopperman, S. Matthews, and H. Pajoohesh, Partial metric spaces, Amer. Math. Monthly 116 (2009), no. 8, 708-718. 56, 67

[43] J. V. Burke and S. Deng, Weak sharp minima revisited. I. Basic theory, Control Cybern. 31 (2002), No. 3, 439-469, Well-posedness in optimization and related topics (Warsaw, 2001). 28

[44] - Weak sharp minima revisited. II. Application to linear regularity and error bounds, Math. Program. 104 (2005), no. 2-3, Ser. B, 235-261. 28

[45] —, Weak sharp minima revisited. III. Error bounds for differentiable convex inclusions, Math. Program. 116 (2009), no. 1-2, Ser. B, 37-56. 28

[46] C.-S. Chuang, L.-J. Lin, and W. Takahashi, Fixed point theorems for single-valued and set-valued mappings on complete metric spaces, J. Nonlinear Convex Anal. 13 (2012), no. 3, 515-527. 80 
[47] S. Cobzaş, Completeness in quasi-metric spaces and Ekeland Variational Principle, Topology Appl. 158 (2011), no. 8, 1073-1084. 2, 33, 34, 35

[48] Ekeland variational principle in asymmetric locally convex spaces, Topology Appl. 159 (2012), no. 10-11, 2558-2569. 38

[49] Functional analysis in asymmetric normed spaces, Frontiers in Mathematics, Birkhäuser/Springer Basel AG, Basel, 2013. 31 32

[50] - Ekeland, Takahashi and Caristi principles in quasi-pseudometric spaces, Topology Appl. 265 (2019), 106831, 22 p. 38

[51] S. Cobzaş, R. Miculescu, and A. Nicolae, Lipschitz functions, Lecture Notes in Mathematics, vol. 2241, Springer, Cham, 2019. 24

[52] E. H. Connell, Properties of fixed point spaces, Proc. Amer. Math. Soc. 10 (1959), 974-979. 8

[53] S. Dancs, M. Hegedüs, and P. Medvegyev, A general ordering and fixed-point principle in complete metric space, Acta Sci. Math. (Szeged) 46 (1983), no. 1-4, 381-388. 25, 26, 39]

[54] A. C. Davis, A characterization of complete lattices, Pacific J. Math. 5 (1955), 311-319. 52

[55] K. Deimling, Nonlinear functional analysis, Springer-Verlag, Berlin, 1985. 4

[56] R. DeMarr, Partially ordered spaces and metric spaces, Amer. Math. Monthly 72 (1965), 628-631. 53 54

[57] M. M. Deza and E. Deza, Encyclopedia of distances, third ed., Springer, Heidelberg, 2014. 78

[58] S. Dhompongsa, W. Inthakon, and A. Kaewkhao, Edelstein's method and fixed point theorems for some generalized nonexpansive mappings, J. Math. Anal. Appl. 350 (2009), no. 1, 12-17. 14

[59] S. Dhompongsa and A. Kaewcharoen, Fixed point theorems for nonexpansive mappings and Suzukigeneralized nonexpansive mappings on a Banach lattice, Nonlinear Anal. 71 (2009), no. 11, 53445353. 14

[60] S. Dhompongsa and H. Yingtaweesittikul, Fixed points for multivalued mappings and the metric completeness, Fixed Point Theory Appl. (2009), Art. ID 972395, 15 pp. 14

[61] M. Edelstein, An extension of Banach's contraction principle, Proc. Amer. Math. Soc. 12 (1961), 7-10. 3

[62] - On fixed and periodic points under contractive mappings, J. London Math. Soc. 37 (1962), 74-79. 3

[63] — A theorem on fixed points under isometries, Amer. Math. Monthly 70 (1963), 298-300. 4

[64] A short proof of a theorem of L. Janos, Proc. Amer. Math. Soc. 20 (1969), 509-510. 4

[65] M. Elekes, On a converse to Banach's fixed point theorem, Proc. Amer. Math. Soc. 137 (2009), no. 9, 3139-3146. 2, 7, 8

[66] A. A. Florinskij, On mappings that are contractive in equivalent metrics, Vestn. Leningr. Univ., Ser. I 1987 (1987), no. 3, 123-124, (Russian, English translation in Vestn. Leningr. Univ., Math. 20, no. 3 (1987), 91-93). [5

[67] - On the existence of connected extensions of metric spaces and the Banach contraction mapping theorem, Vestn. Leningr. Univ., Ser. I 1991 (1991), no. 4, 18-22, (Russian, English translation in Vestn. Leningr. Univ., Math. 24, no. 4 (1991), 17-20). 6]

[68] Connected extension of metric spaces and fixed points of Banach contractions, Probl. Mat. Anal. 17 (1997), 227-237, (Russian, English translation in J. Math. Sci. (NY) 97, no. 4 (1999), 4329-4335). 5

[69] O. Frink, Topology in lattices, Trans. Amer. Math. Soc. 51 (1942), 569-582. 49, 50 52

[70] J. García-Falset, E. Llorens-Fuster, and T. Suzuki, Fixed point theory for a class of generalized nonexpansive mappings, J. Math. Anal. Appl. 375 (2011), no. 1, 185-195. 14

[71] P. G. Georgiev, The strong Ekeland variational principle, the strong drop theorem and applications, J. Math. Anal. Appl. 131 (1988), no. 1, 1-21. 18

[72] P. Ghosh and A. Deb Ray, A characterization of completeness of generalized metric spaces using generalized Banach contraction principle, Demonstratio Math. 45 (2012), no. 3, 717-724. 80

[73] G. Gierz, K. H. Hofmann, K. Keimel, J. D. Lawson, M. Mislove, and D. S. Scott, Continuous lattices and domains, Encyclopedia of Mathematics and its Applications, vol. 93, Cambridge University Press, Cambridge, 2003. 50

[74] K. Goebel and W. A. Kirk, Topics in metric fixed point theory, Cambridge Studies in Advanced Mathematics, vol. 28, Cambridge University Press, Cambridge, 1990. 14 
[75] J. Goubault-Larrecq, Non-Hausdorff topology and domain theory: Selected topics in point-set topology, New Mathematical Monographs, vol. 22, Cambridge University Press, Cambridge, 2013. 45, 46. $49,50,51$

[76] M. Grabiec, Y. J. Cho, and R. Saadati, Completeness and fixed poitns in probabilistic quasi-pseudometric spaces, Bull. Stat. Econ. 2 (2008), no. A08, 39-47. 80

[77] A. Granas and J. Dugundji, Fixed point theory, Springer Monographs in Mathematics, SpringerVerlag, New York, 2003. 52

[78] R. H. Haghi, Sh. Rezapour, and N. Shahzad, Be careful on partial metric fixed point results, Topology Appl. 160 (2013), no. 3, 450-454. 68

[79] S. Han, J. Wu, and D. Zhang, Properties and principles on partial metric spaces, Topology Appl. 230 (2017), 77-98. 63,64

[80] P. Hitzler and A. K. Seda, Dislocated topologies, J. Electr. Eng. 51 (2000), no. 12/s, 3-7. 76, 77, 78

[81] _ A "converse" of the Banach contraction mapping theorem, J. Electr. Eng. 52 (2001), no. 10/s, 3-6. 7

[82] The fixed-point theorems of Priess-Crampe and Ribenboim in logic programming, Valuation theory and its applications, Vol. I (Saskatoon, SK, 1999), Fields Inst. Commun., vol. 32, Amer. Math. Soc., Providence, RI, 2002, pp. 219-235. 7

[83] S. B. Hosseini and R. Saadati, Completeness results in probabilistic metric spaces. I, Commun. Appl. Anal. 9 (2005), no. 3-4, 549-553. 80

[84] P. Howard and J. E. Rubin, Consequences of the axiom of choice, Mathematical Surveys and Monographs, vol. 59, American Mathematical Society, Providence, RI, 1998, With 1 IBM-PC floppy disk (3.5 inch; WD). 51

[85] T. K. Hu, On a fixed-point theorem for metric spaces, Amer. Math. Monthly 74 (1967), 436-437. 9]

[86] H. Huang, Global weak sharp minima and completeness of metric space, Acta Math. Sci. Ser. B Engl. Ed. 25 (2005), no. 2, 359-366. 28

[87] S. Iemoto, W. Takahashi, and H. Yingtaweesittikul, Nonlinear operators, fixed points and completeness of metric spaces, Fixed point theory and its applications, Yokohama Publ., Yokohama, 2010, pp. 93-101. 80

[88] A. A. Ivanov, Fixed points of mappings of metric spaces, Studies in topology, II, Zap. Naučn. Sem. Leningrad. Otdel. Mat. Inst. Steklov. (LOMI) 66 (1976), 5-102, 207 (Russian). 4

[89] J. Jachymski, An iff fixed point criterion for continuous self-mappings on a complete metric space, Aequationes Math. 48 (1994), no. 2-3, 163-170. 4

[90] Some consequences of fundamental ordering principles in metric fixed point theory, Ann. Univ. Mariae Curie-Skłodowska Sect. A 51 (1997), no. 2, 123-134, Proceedings of Workshop on Fixed Point Theory (Kazimierz Dolny, 1997). 54

[91] - Fixed point theorems in metric and uniform spaces via the Knaster-Tarski principle, Nonlinear Anal. 32 (1998), no. 2, 225-233. 54

[92] - Some consequences of the Tarski-Kantorovitch ordering theorem in metric fixed point theory, Quaestiones Math. 21 (1998), no. 1-2, 89-99. 54

[93] A short proof of the converse to the contraction principle and some related results, Topol. Methods Nonlinear Anal. 15 (2000), no. 1, 179-186, Dedicated to Juliusz Schauder, 1899-1943. 4

[94] - Order-theoretic aspects of metric fixed point theory, Handbook of metric fixed point theory, Kluwer Acad. Publ., Dordrecht, 2001, pp. 613-641. [51] 54]

[95] Converses to fixed point theorems of Zermelo and Caristi, Nonlinear Anal. 52 (2003), no. 5, 1455-1463. 52

[96] _ Equivalent conditions for generalized contractions on (ordered) metric spaces, Nonlinear Anal. 74 (2011), no. 3, 768-774. 56

[97] A stationary point theorem characterizing metric completeness, Appl. Math. Lett. 24 (2011), no. 2, 169-171. 14

[98] S. Janković, Z. Kadelburg, and S. Radenović, On cone metric spaces: a survey, Nonlinear Anal. 74 (2011), no. 7, 2591-2601. 68

[99] L. Janoš, A converse of Banach's contraction theorem, Proc. Amer. Math. Soc. 18 (1967), $287-289$. 4

[100] A converse of the generalized Banach's contraction theorem, Arch. Math. (Basel) 21 (1970), 69-71. 4 
[101] G.-J. Jiang, On characterization of metric completeness, Turkish J. Math. 24 (2000), no. 3, $267-272$. 14,15

[102] O. Kada, T. Suzuki, and W. Takahashi, Nonconvex minimization theorems and fixed point theorems in complete metric spaces, Math. Japon. 44 (1996), no. 2, 381-391. 24] 78, 80]

[103] E. Karapinar, Generalizations of Caristi Kirk's theorem on partial metric spaces, Fixed Point Theory Appl. (2011), 2011:4, 7 pp. 72

[104] E. Karapinar and S. Romaguera, On the weak form of Ekeland's variational principle in quasi-metric spaces, Topology Appl. 184 (2015), 54-60. 2, 36. 38

[105] E. Karapınar and P. Salimi, Dislocated metric space to metric spaces with some fixed point theorems, Fixed Point Theory Appl. (2013), 2013:222, 19 pp. 78

[106] S. Kasahara, Classroom Notes: A remark on the converse of Banach's Contraction Theorem, Amer. Math. Monthly 75 (1968), no. 7, 775-776. 4

[107] K. Keimel, Topological cones: functional analysis in a $\mathrm{T}_{0}$-setting, Semigroup Forum 77 (2008), no. 1, 109-142. 50

[108] Weak topologies and compactness in asymmetric functional analysis, Topology Appl. $\mathbf{1 8 5 / 1 8 6}$ (2015), 1-22. 50

[109] J. L. Kelley, General topology. 2nd ed., Graduate Texts in Mathematics, vol. 27, Springer-Verlag, New York - Heidelberg - Berlin, 1975. 50, 59, 77

[110] J. C. Kelly, Bitopological spaces, Proc. London Math. Soc. (3) 13 (1963), 71-89. 31

[111] M. A. Khamsi, Generalized metric spaces: A survey, J. Fixed Point Theory Appl. 17 (2015), no. 3, 455-475. 55

[112] W. Kirk and N. Shahzad, Fixed point theory in distance spaces, Springer, Cham, 2014. 56, 70, 78, 80

[113] W. A. Kirk, Contraction mappings and extensions, Handbook of metric fixed point theory, Kluwer Acad. Publ., Dordrecht, 2001, pp. 1-34. 3 4

[114] W. A. Kirk and L. M. Saliga, The Brézis-Browder order principle and extensions of Caristi's theorem, Nonlinear Anal. 47 (2001), no. 4, 2765-2778. 41

[115] W. A. Kirk and B. Sims (eds.), Handbook of metric fixed point theory, Kluwer Academic Publishers, Dordrecht, 2001. 14]

[116] V. L. Klee, Jr., Some topological properties of convex sets, Trans. Amer. Math. Soc. 78 (1955), 30-45. 8

[117] J. Klimeš, Characterizations of completeness for semilattices by using of fixed points, Scripta Fac. Sci. Natur. Univ. Purk. Brun. 12 (1982), no. 10, 507-513. 55

[118] _ A characterization of a semilattice completeness, Scripta Fac. Sci. Natur. Univ. Purk. Brun. 14 (1984), no. 8, 399-407. 55

[119] Fixed point characterization of completeness on lattices for relatively isotone mappings, Arch. Math. (Brno) 20 (1984), no. 3, 125-132. 51, 54

[120] A characterization of inductive posets, Arch. Math. (Brno) 21 (1985), no. 1, 39-42. 555

[121] R. D. Kopperman, All topologies come from generalized metrics, Amer. Math. Monthly 95 (1988), No.2, 89-97. 1

[122] W Which topologies are quasimetrizable?, Topology Appl. 52 (1993), no. 2, 99-107. 31

[123] B. K. Lahiri, M. K. Chakrabarty, and A. Sen, Converse of Banach's contraction principle and star operation, Proc. Nat. Acad. Sci. India Sect. A 79 (2009), no. 4, 367-374. 4

[124] S. Leader, A topological characterization of Banach contractions, Pacific J. Math. 69 (1977), no. 2, 461-466. 5

[125] _ Uniformly contractive fixed points in compact metric spaces, Proc. Amer. Math. Soc. 86 (1982), no. 1, 153-158. 5

[126] Equivalent Cauchy sequences and contractive fixed points in metric spaces, Studia Math. 76 (1983), no. 1, 63-67. 5

[127] W. Lee and Y. Choi, A survey on characterizations of metric completeness, Nonlinear Anal. Forum 19 (2014), 265-276. 2,8

[128] L.-J. Lin and W.-S. Du, Ekeland's variational principle, minimax theorems and existence of nonconvex equilibria in complete metric spaces, J. Math. Anal. Appl. 323 (2006), no. 1, 360-370. 79

[129] Some equivalent formulations of the generalized Ekeland's variational principle and their applications, Nonlinear Anal. 67 (2007), no. 1, 187-199. 79

[130] _ On maximal element theorems, variants of Ekeland's variational principle and their applications, Nonlinear Anal. 68 (2008), no. 5, 1246-1262.79] 
[131] Z. Liu, Fixed points and completeness, Turkish J. Math. 20 (1996), no. 4, 467-472. 14]

[132] Z. Liu and S. M. Kang, On characterizations of metric completeness, Indian J. Math. 44 (2002), no. 2, 183-187. 14]

[133] - On characterizations of $\leq$-completeness and metric completeness, Southeast Asian Bull. Math. 27 (2003), no. 2, 325-331. 14

[134] R. Mańka, Connection between set theory and the fixed point property, Colloq. Math. 53 (1987), no. 2, 177-184. 51

[135] _ Some forms of the axiom of choice, Jbuch. Kurt-Gödel-Ges. (1988), 24-34. 51]

[136] _ Turinici's fixed point theorem and the axiom of choice, Rep. Math. Logic (1988), no. 22, 15-19 (1989). 51

[137] J. Marín, S. Romaguera, and P. Tirado, Weakly contractive multivalued maps and w-distances on complete quasi-metric spaces, Fixed Point Theory Appl. (2011), 2011:2, 9 pp. 80.

[138] G. Markowsky, Chain-complete posets and directed sets with applications, Algebra Universalis 6 (1976), no. 1, 53-68. 51

[139] S. G. Matthews, The cycle contraction mapping theorem, Research Report, no. 228, University of Warwick, UK, 1992, 17 p. 56

[140] _ Partial metric spaces, Research Report, no. 212, University of Warwick, UK, 1992, 21 p. 56. 6267

[141] The topology of partial metric spaces, Research Report, no. 222, University of Warwick, UK, 1992, 20 p. 56 65]

[142] _ Partial metric topology, Papers on general topology and applications (Flushing, NY, 1992), Ann. New York Acad. Sci., vol. 728, New York Acad. Sci., New York, 1994, pp. 183-197. 56, 62, 67

[143] P. R. Meyers, Some extensions of Banach's contraction theorem, J. Res. Nat. Bur. Standards Sect. B 69B (1965), 179-184. 5

[144] _ A converse to Banach's contraction theorem, J. Res. Nat. Bur. Standards Sect. B 71B (1967), 73-76. [5]

[145] R. N. Mukherjee and T. Som, An application of Meyer's theorem on converse of Banach's contraction principle, Bull. Inst. Math. Acad. Sinica 12 (1984), no. 3, 253-255. [5]

[146] V. V. Nemytskiu,, The fixed point method in analysis, Uspekhi Mat. Nauk 1 (1936), 141-174 (Russian). 4

[147] A.-M. Nicolae, On completeness and fixed points, Babeş-Bolyai University, Faculty of mathematics and Computer Science, Cluj-Napoca, 2008, Master Thesis. 8

[148] C. P. Niculescu and L.-E. Persson, Convex functions and their applications. A contemporary approach, CMS Books in Mathematics/Ouvrages de Mathématiques de la SMC, Springer, Cham, 2018, (2nd edition). 19

[149] J. J. Nieto, R. L. Pouso, and R. Rodríguez-López, Fixed point theorems in ordered abstract spaces, Proc. Amer. Math. Soc. 135 (2007), no. 8, 2505-2517. 56

[150] J. J. Nieto and R. Rodríguez-López, Contractive mapping theorems in partially ordered sets and applications to ordinary differential equations, Order 22 (2005), no. 3, 223-239 (2006). 55

[151] Existence and uniqueness of fixed point in partially ordered sets and applications to ordinary differential equations, Acta Math. Sin. (Engl. Ser.) 23 (2007), no. 12, 2205-2212. 56

[152] K. F. Ng and X. Y. Zheng, Global weak sharp minima on Banach spaces, SIAM J. Control Optim. 41 (2003), no. 6, 1868-1885. 28, 30, 31]

[153] S. Oltra and O. Valero, Banach's fixed point theorem for partial metric spaces, Rend. Istit. Mat. Univ. Trieste 36 (2004), no. 1-2, 17-26 (2005). 62

[154] S. J. O'Neill, Two topologies are better than one, Preprint, University of Warwick, 1995. 66, 68

[155] _ Partial metrics, valuations, and domain theory, Ann. New York Acad. Sci. 806 (1996), 304-315, Papers on general topology and applications (Gorham, ME, 1995). 62

[156] V. I. Opoitsev, A converse of the contraction mapping principle, Uspekhi Mat. Nauk 31 (1976), no. 4 (190), 169-198 (Russian). 4

[157] D. Paesano and P. Vetro, Suzuki's type characterizations of completeness for partial metric spaces and fixed points for partially ordered metric spaces, Topology Appl. 159 (2012), no. 3, 911-920. 13

[158] B. Palczewski and A. Miczko, On some converses of generalized Banach contraction principles, Nonlinear functional analysis and its applications (Maratea, 1985), NATO Adv. Sci. Inst. Ser. C Math. Phys. Sci., vol. 173, Reidel, Dordrecht, 1986, pp. 335-351. 4 
[159] - Converses of generalized Banach contraction principles and remarks on mappings with a contractive iterate at the point, Univ. u Novom Sadu Zb. Rad. Prirod.-Mat. Fak. Ser. Mat. 17 (1987), no. 1, 71-91. 4

[160] S. Park and B. E. Rhoades, Comments on characterizations for metric completeness, Math. Japon. 31 (1986), no. 1, 95-97. 80

[161] L. Pasicki, Dislocated metric and fixed point theorems, Fixed Point Theory Appl. (2015), 2015:82, 14 pp. 77,78

[162] J.-P. Penot, The drop theorem, the petal theorem and Ekeland's variational principle, Nonlinear Anal. 10 (1986), no. 9, 813-822. 15, 17

[163] C. Petalas and T. Vidalis, A fixed point theorem in non-Archimedean vector spaces, Proc. Amer. Math. Soc. 118 (1993), no. 3, 819-821. 7

[164] A. Petruşel, Multivalued weakly Picard operators and applications, Sci. Math. Jpn. 59 (2004), no. 1, 169-202. 26

[165] A. Petruşel and G. Petruşel, Multivalued Picard operators, J. Nonlinear Convex Anal. 13 (2012), no. $1,157-171.26$

[166] A. Petruşel and I. A. Rus, Fixed point theorems in ordered L-spaces, Proc. Amer. Math. Soc. 134 (2006), no. 2, 411-418. 56

[167] R. R. Phelps, Convex functions, monotone operators and differentiability, second ed., Lecture Notes in Mathematics, vol. 1364, Springer-Verlag, Berlin, 1993. 15

[168] S. Prieß-Crampe, Der Banachsche Fixpunktsatz für ultrametrische Räume, Results Math. 18 (1990), no. 1-2, 178-186. 7

[169] A. C. M. Ran and M. C. B. Reurings, A fixed point theorem in partially ordered sets and some applications to matrix equations, Proc. Amer. Math. Soc. 132 (2004), no. 5, 1435-1443. 55, 56

[170] S. Romaguera, A Kirk type characterization of completeness for partial metric spaces, Fixed Point Theory Appl. (2010), Art. ID 493298, 6 pp. 20, 69, 70, 72

[171] S. Romaguera and P. Tirado, A characterization of Smyth complete quasi-metric spaces via Caristi's fixed point theorem, Fixed Point Theory Appl. (2015), 2015:183, 13 pp. 38

[172] S. Romaguera and O. Valero, Domain theoretic characterisations of quasi-metric completeness in terms of formal balls, Math. Structures Comput. Sci. 20 (2010), no. 3, 453-472. 38

[173] H. Rubin and J. E. Rubin, Equivalents of the axiom of choice. II, Studies in Logic and the Foundations of Mathematics, vol. 116, North-Holland Publishing Co., Amsterdam, 1985. 51

[174] I. A. Rus, Metrical fixed point theorems, Universitatea "Babeş-Bolyai", Facultatea de Matematica, Cluj-Napoca, 1979. 4

[175] — Weakly Picard mappings, Comment. Math. Univ. Carolin. 34 (1993), no. 4, 769-773. 4

[176] _ Generalized contractions and applications, Cluj University Press, Cluj-Napoca, 2001. 4

[177] _ Picard operators and applications, Sci. Math. Jpn. 58 (2003), no. 1, 191-219. 5

[178] _ Fixed point theory in partial metric spaces, An. Univ. Vest Timiş. Ser. Mat.-Inform. 46 (2008), no. 2, 149-160. 62

[179] I. A. Rus, A. Petruşel, and G. Petruşel, Fixed point theory, Cluj University Press, Cluj-Napoca, 2008. 4, 5, $9,56,78$

[180] B. Samet, Discussion on "A fixed point theorem of Banach-Caccioppoli type on a class of generalized metric spaces" by A. Branciari, Publ. Math. Debrecen 76 (2010), no. 3-4, 493-494. 80

[181] D. Scott, Continuous lattices, Toposes, algebraic geometry and logic (Conf., Dalhousie Univ., Halifax, N. S., 1971), Springer, Berlin, 1972, pp. 97-136. Lecture Notes in Math., Vol. 274. [50]

[182] N. Shahzad and O. Valero, On 0-complete partial metric spaces and quantitative fixed point techniques in denotational semantics, Abstr. Appl. Anal. (2013), Art. ID 985095, 11 pp. 62

[183] _ A Nemytskii-Edelstein type fixed point theorem for partial metric spaces, Fixed Point Theory Appl. (2015), 2015:26, 15 pp. 62

[184] N. Shioji, T. Suzuki, and W. Takahashi, Contractive mappings, Kannan mappings and metric completeness, Proc. Amer. Math. Soc. 126 (1998), no. 10, 3117-3124. 80

[185] R. E. Smithson, Fixed points of order preserving multifunctions, Proc. Amer. Math. Soc. 28 (1971), 304-310. 51

[186] _ Fixed points in partially ordered sets, Pacific J. Math. 45 (1973), 363-367. 52

[187] V. Stoltenberg-Hansen, I. Lindström, and E. R. Griffor, Mathematical theory of domains, Cambridge Tracts in Theoretical Computer Science, vol. 22, Cambridge University Press, Cambridge, 1994. 50

[188] P. V. Subrahmanyam, Completeness and fixed-points, Monatsh. Math. 80 (1975), no. 4, 325-330. 9 
[189] F. Sullivan, A characterization of complete metric spaces, Proc. Amer. Math. Soc. 83 (1981), no. 2, 345-346. 16

[190] Ordering and completeness of metric spaces, Nieuw Arch. Wisk. (3) 29 (1981), no. 2, 178193. 2, 8,16

[191] T. Suzuki, Generalized distance and existence theorems in complete metric spaces, J. Math. Anal. Appl. 253 (2001), no. 2, 440-458. 78, 79]

[192] Several fixed point theorems concerning $\tau$-distance, Fixed Point Theory Appl. (2004), no. 3, 195-209. 79

[193] - Counterexamples on $\tau$-distance versions of generalized Caristi's fixed point theorems, Bull. Kyushu Inst. Technol. Pure Appl. Math. (2005), no. 52, 15-20. 79

[194] _ The strong Ekeland variational principle, J. Math. Anal. Appl. 320 (2006), no. 2, 787-794. 18, 19, 79

[195] - Fixed point theorems and convergence theorems for some generalized nonexpansive mappings, J. Math. Anal. Appl. 340 (2008), no. 2, 1088-1095. 13 14

[196] _ A generalized Banach contraction principle that characterizes metric completeness, Proc. Amer. Math. Soc. 136 (2008), no. 5, 1861-1869. 13

[197] $-w$-distances and $\tau$-distances, Nonlinear Funct. Anal. Appl. 13 (2008), no. 1, 15-27. 79

[198] - Some notes on $\tau$-distance versions of Ekeland's variational principle, Bull. Kyushu Inst. Technol. Pure Appl. Math. (2009), no. 56, 19-28. 79]

[199] S Some notes on $\tau$-distance versions of Ekeland's variational principle, Bull. Kyushu Inst. Technol. Pure Appl. Math. (2009), no. 56, 19-28. 79

[200] _ Characterizations of reflexivity and compactness via the strong Ekeland variational principle, Nonlinear Anal. 72 (2010), no. 5, 2204-2209. 18, 21, 23, 24

[201] _ Some notes on the class of contractions with respect to $\tau$-distance, Bull. Kyushu Inst. Technol. Pure Appl. Math. (2010), no. 57, 9-18. 79

[202] T. Suzuki and W. Takahashi, Fixed point theorems and characterizations of metric completeness, Topol. Methods Nonlinear Anal. 8 (1996), no. 2, 371-382 (1997). 80

[203] W. Takahashi, Existence theorems generalizing fixed point theorems for multivalued mappings, Fixed point theory and applications (Marseille, 1989), Pitman Res. Notes Math. Ser., vol. 252, Longman Sci. Tech., Harlow, 1991, pp. 397-406. 24

[204] Existence theorems in metric spaces and characterizations of metric completeness, NLA98: Convex analysis and chaos (Sakado, 1998), Josai Math. Monogr., vol. 1, Josai Univ., Sakado, 1999, pp. $67-85.80$

[205] , Nonlinear functional analysis. fixed point theory and its applications, Yokohama Publishers, Yokohama, 2000. 24, 80

[206] W. Takahashi, N.-C. Wong, and J.-C. Yao, Fixed point theorems for general contractive mappings with $W$-distances in metric spaces, J. Nonlinear Convex Anal. 14 (2013), no. 3, 637-648. 80

[207] M. R. Tasković, The axiom of choice, fixed point theorems, and inductive ordered sets, Proc. Amer. Math. Soc. 116 (1992), no. 4, 897-904. 51

[208] Axiom of choice-100th next, Math. Morav. 8 (2004), no. 1, 39-62. 51]

[209] The axiom of infinite choice, Math. Morav. 16 (2012), no. 1, 1-32. 51

[210] D. Tataru, Viscosity solutions of Hamilton-Jacobi equations with unbounded nonlinear terms, J. Math. Anal. Appl. 163 (1992), no. 2, 345-392. 79

[211] M. Turinici, Strong variational principles and generic well-posedness, Demonstr. Math. 38 (2005), no. 4, 935-941. 18

[212] O. Valero, On Banach fixed point theorems for partial metric spaces, Appl. Gen. Topol. 6 (2005), no. 2, 229-240. 62

[213] _ On Banach's fixed point theorem and formal balls, Appl. Sci. 10 (2008), 256-258. 62

[214] G. Wang, B. L. Chen, and L. S. Wang, A new converse to Banach's contraction mapping theorem: a nonlinear convergence principle, Gongcheng Shuxue Xuebao 16 (1999), no. 1, 135-138 (Chinese). 4

[215] L. E. Ward, Jr., Completeness in semi-lattices, Canad. J. Math. 9 (1957), 578-582. 52

[216] J. D. Weston, A characterization of metric completeness, Proc. Amer. Math. Soc. 64 (1977), no. 1, 186-188. 16

[217] E. Witt, On Zorn's theorem, Rev. Mat. Hisp.-Amer., IV. Ser. 10 (1950), 82-85 (Spanish). 51

[218] E. S. Wolk, Dedekind completeness and a fixed-point theorem, Canad. J. Math. 9 (1957), 400-405. 52 
[219] J. S. W. Wong, Generalizations of the converse of the contraction mapping principle, Canad. J. Math. 18 (1966), 1095-1104. 4

[220] S.-W. Xiang, Equivalence of completeness and contraction property, Proc. Amer. Math. Soc. 135 (2007), no. 4, 1051-1058. 12,13

[221] E. Zeidler, Nonlinear functional analysis and its applications. I. Fixed-point theorems, SpringerVerlag, New York, 1986, Translated from the German by Peter R. Wadsack. 51]

Babeş-Bolyai University, Faculty of Mathematics and Computer Science, 400084 Cluj-Napoca, Romania E-mail address: scobzas@math.ubbcluj.ro 